\title{
Methods for Measuring Cancer Disparities: Using Data Relevant to Healthy People 2010 Cancer-Related Objectives
}

Sam Harper

John Lynch

Center for Social Epidemiology and Population Health

University of Michigan

Current contact information:

Department of Epidemiology, Biostatistics and Occupational Health

McGill University, Purvis Hall

Montreal QC H3A 1A2

Email: sam.harper@mcgill.ca / john.lynch@mcgill.ca

Phone: (514) 398-6261

Fax: (514) 398-4266

This report was written under contract from the Surveillance Research Program (SRP) and the Applied Research Program (ARP) of the Division of Cancer Control and Population Sciences of the National Cancer Institute, NIH. Additional support was provided by the Office of Disease Prevention in the Office of the Director at the National Institutes of Health. It represents the interests of these organizations in health disparities related to cancer, quantitative assessment and monitoring of these disparities, and interventions to remove them. NCI Project Officers for this contract are Marsha E. Reichman, Ph.D. (SRP), Bryce Reeve, Ph.D. (ARP), and Nancy Breen, Ph.D. (ARP). 


\section{Table of Contents}

Executive Summary

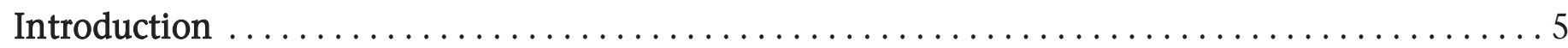

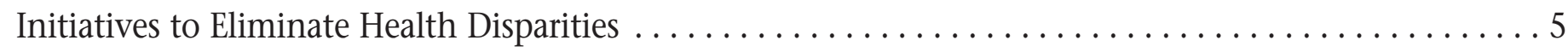

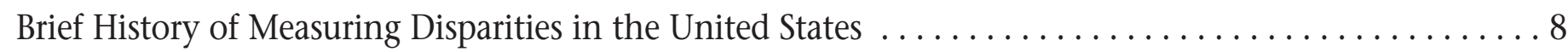

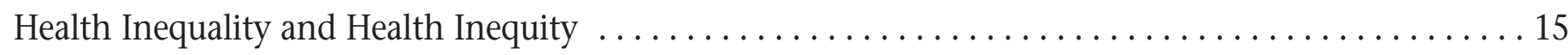

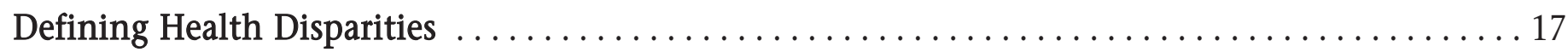

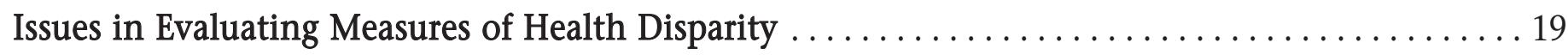

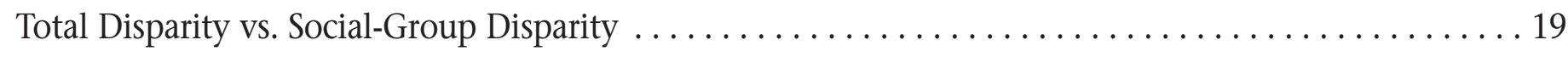

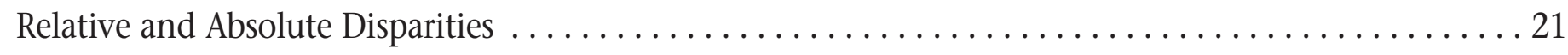

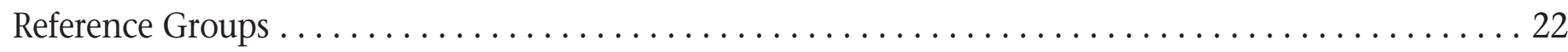

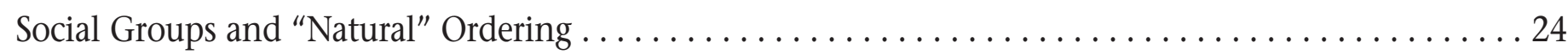

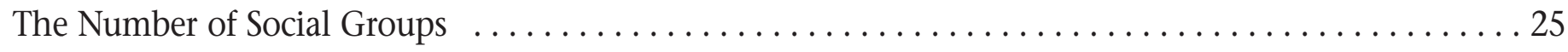

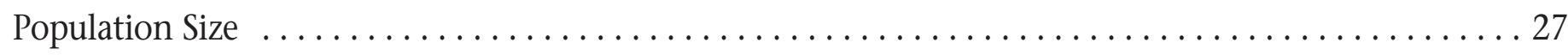

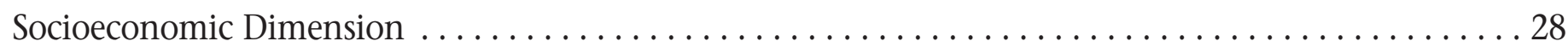

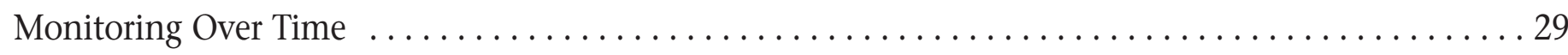

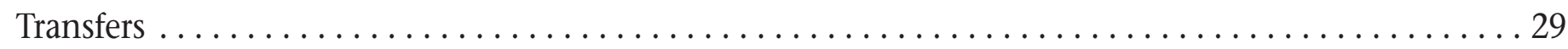

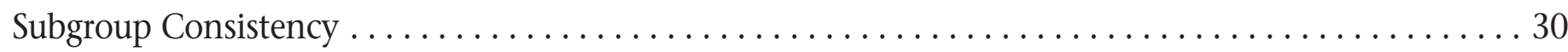

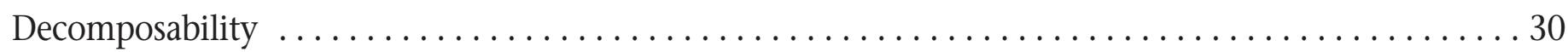

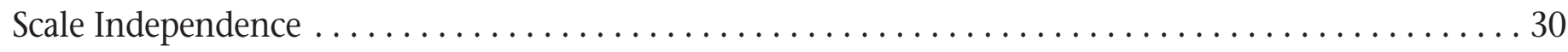

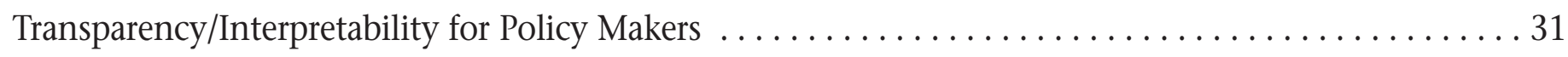




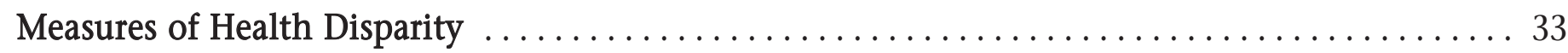

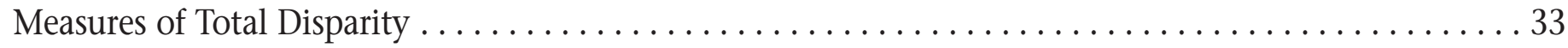

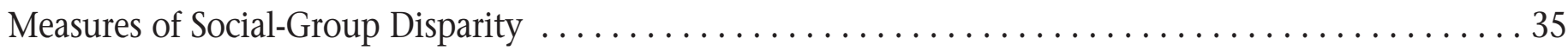

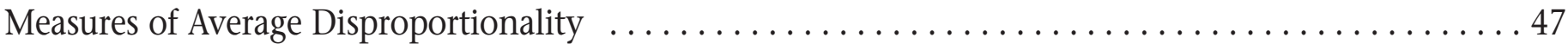

Choosing a Suite of Health Disparity Indicators $\ldots \ldots \ldots \ldots \ldots \ldots \ldots \ldots \ldots \ldots \ldots \ldots \ldots \ldots \ldots \ldots \ldots \ldots \ldots$

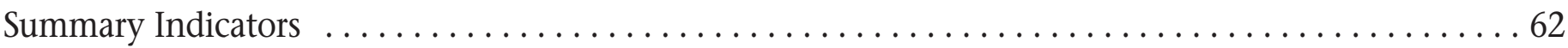

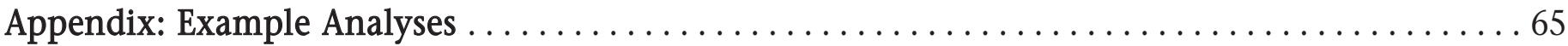

References .................................................... 75

\section{Figures}

Figure S1. Absolute and Relative Gender Disparity in Stomach Cancer Mortality, 1930-2000 . . . . . . 1

Figure S2. Proportion of Women Age 40 and Over Who Did Not Receive a Mammogram in the

Past 2 Years by Level of Educational Achievement, 1990-2002, Trends in Absolute and

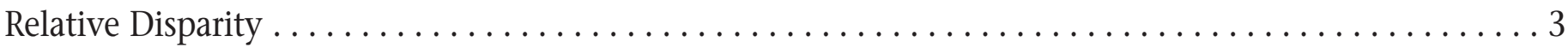

Figure 1. Lung Cancer Mortality, Females, U.S., $1995-1999$. . . . . . . . . . . . . . . . . . . 7

Figure 2. Lung Cancer Incidence by Gender and Race/Ethnicity, 1992-1999 . . . . . . . . . . . . . . 8

Figure 3. Mean and 10th-90th Percentiles of Body Mass Index by Education, NHIS, 1997 . . . . . . . . 20

Figure 4. Hypothetical Distributions of Life Expectancy in Two Populations . ................. 21

Figure 5. Absolute and Relative Gender Disparity in Stomach Cancer Mortality, 1930-2000 . . . . . . . 22

Figure 6. Relative Risk (RR) of Incident Cervical Cancer Among Hispanics According to Varying

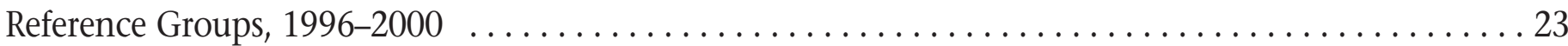

Figure 7. Age-Adjusted Incidence of Kidney/Renal Pelvis Cancer and Myeloma by Race and Ethnicity, 1996-2000 ....................................................... 25

Figure 8. Proportion of Men Reporting Recent Use of Screening Fecal Occult Blood Tests (FOBT),

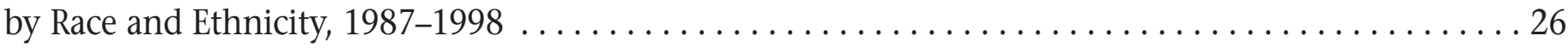

Figure 9. Percent Change in Population Size by Race and Hispanic Origin, 1980-2000 . . . . . . . . 28

Figure 10. Absolute and Relative Black-White Disparities in Prostate and Stomach Cancer Incidence, 1992-1999 ....................................................... 36

Figure 11. Example of a Simple Regression-Based Disparity Measure $\ldots \ldots \ldots \ldots \ldots \ldots \ldots \ldots \ldots$ 
Figure 12. Income-Based Slope Index of Inequality for Current Smoking, NHIS, $2002 \ldots \ldots \ldots \ldots \ldots$

Figure 13. Example of the Population-Attributable Risk Percent $\ldots \ldots \ldots \ldots \ldots \ldots \ldots \ldots \ldots \ldots 2$

Figure 14. Disparity in Mammography Screening Among Racial/Ethnic Groups, NHIS, 2000 . . . ... 45

Figure 15. Age-Adjusted Lung Cancer Mortality by U.S. Census Division, 1968-1998 . . . . . . . . . . . 46

Figure 16. Example of the "Disproportionality" of Deaths and Population, by Gender and Education,

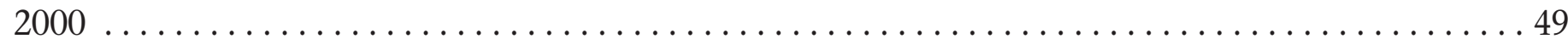

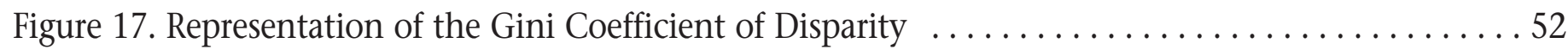

Figure 18. Representation of the Health Concentration Curve $\ldots \ldots \ldots \ldots \ldots \ldots \ldots \ldots \ldots \ldots \ldots$

Figure 19. Relative Concentration Curves for Educational Disparity in Obesity in New York State,

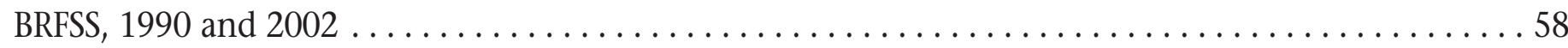

Figure 20. Absolute Concentration Curves for Educational Disparity in Obesity in New York State,

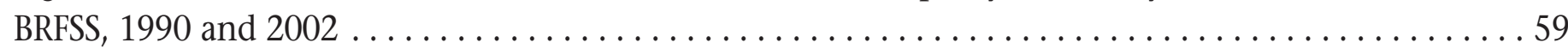

Figure A1. Proportion of Women Age 40 and Over Who Did Not Receive a Mammogram in the

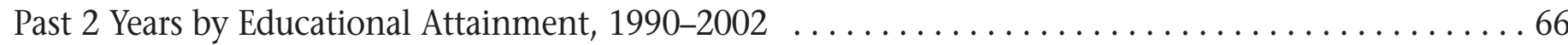

Figure A2. Trends in Education-Related Disparity and Prevalence for the Proportion of Women Age 40 and Over Who Did Not Receive a Mammogram in the Past 2 Years, 1990-2002 . . . . . . . . . 69

Figure A3. Trends in Mortality from Colorectal Cancer by Race, Ages 45-64, 1990-2001 . . . . . . . . . 71 Figure A4. Racial Disparity Trends in Working-Age (45-64) Mortality from Colorectal Cancer

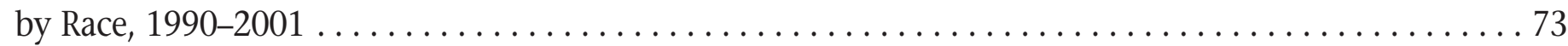

\section{Tables}

Table 1. Incidence of Esophageal Cancer, Ages 25-64 by Race, 12 SEER Registries, 1992-2000 . . . . . . 44 Table 2. Commonly Used Disproportionality Functions $\ldots \ldots \ldots \ldots \ldots \ldots \ldots \ldots \ldots \ldots$

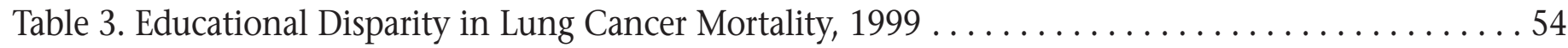
Table 4. Example of Extended Relative and Absolute Concentration Index Applied to the Change in Educational Disparity in Current Smoking, Michigan, 1990 and 2002 ..................... 56

Table 5. Summary Table of Advantages and Disadvantages of Potential Health Disparity Measures ... . 64 Table A1. Example of Relative and Absolute Concentration Index Applied to the Change in

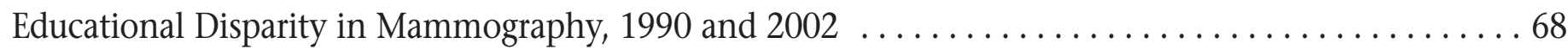
Table A2. Example of Theil Index and the Between-Group Variance Applied to the Change in Racial Disparity in Colorectal Cancer Mortality, 1990 and 2001 



\section{Executive Summary}

Healthy People 2010 has two overarching goals: to increase the span of healthy life and to eliminate health disparities across the categories of gender, race or ethnicity, education or income, disability, geographic location, and sexual orientation (1). This report raises some conceptual issues and reviews different methodological approaches germane to measuring progress toward the goal of eliminating cancer-related health disparities (2). Despite the increased attention to social disparities in health, no clear framework exists to define and measure health disparities. This may create confusion in communicating the extent of cancer-related health disparities and hinder the ability of public health organizations to monitor progress toward the Healthy People 2010 cancer objectives. The recommendations in this report are based on the following considerations:
- Choosing a particular measure of health disparity reflects, implicitly or explicitly, different perspectives about what quantities or characteristics of health disparity are thought to be important to capture. For instance, most research in health disparities is based on relative comparisons (e.g., a ratio of rates), but it is equally appropriate to make absolute comparisons (e.g., the arithmetic difference between rates). Figure S1 shows male/female disparities in stomach cancer mortality during the $20^{\text {th }}$ century. If we use an absolute comparison (arithmetic difference in rates), disparities have declined since about 1950; if we use a relative comparison (ratio of rates), they have increased almost continuously. This is an example of how the same underlying data potentially could generate two divergent interpretations of trends in cancer-related health

\section{Figure S1. Absolute and Relative Gender Disparity in Stomach Cancer Mortality, 1930-2000}

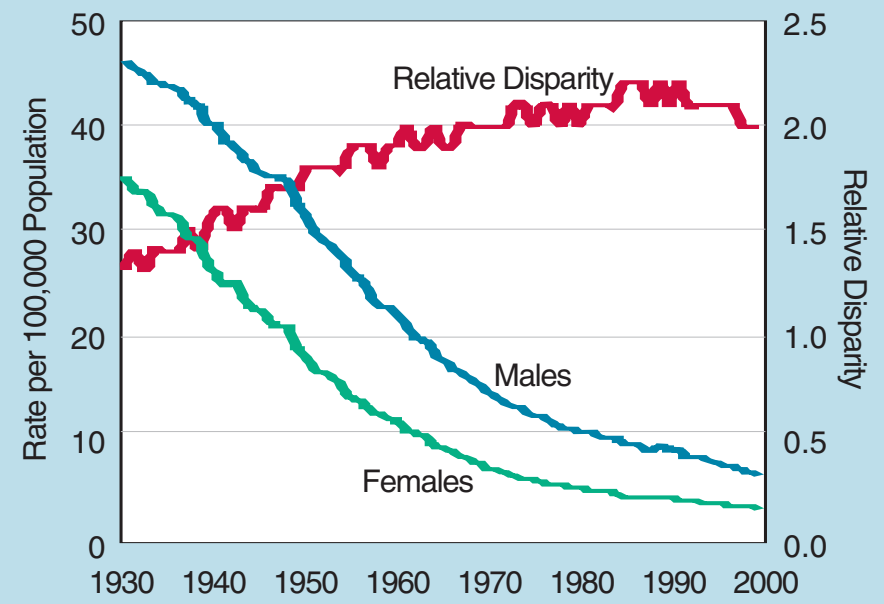

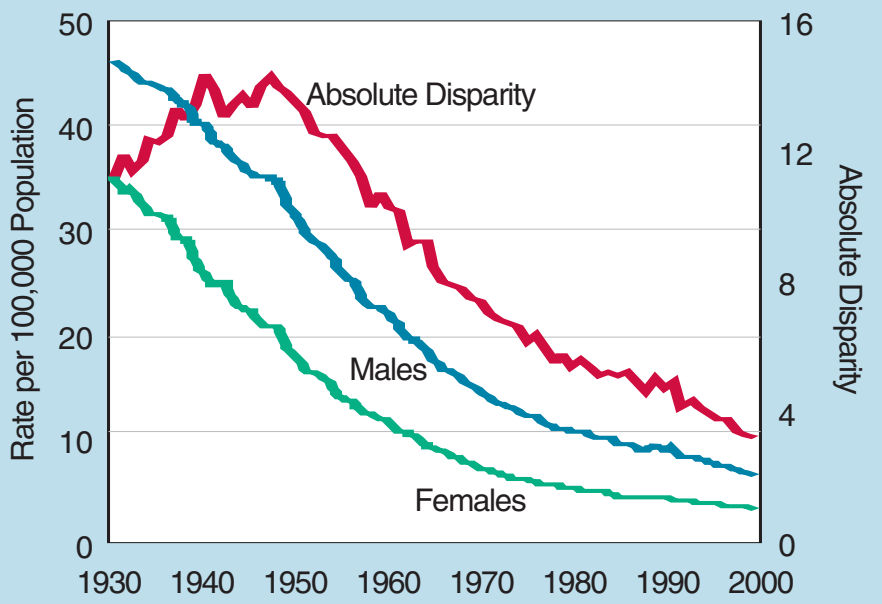


outcomes-dependent on which measure of disparity is used.

- In this report, we adopt a "population health" perspective on health disparities. A population health perspective reflects a primary concern for the total population health burden of disparities by considering the number of cases of the cancerrelated health outcome (e.g., mortality, incidence, screening, etc.) that would be reduced or eliminated by an intervention. This perspective emphasizes absolute differences between groups and the size of the population subgroups involved. We believe that such an approach offers a justifiable basis on which to assess the total population burden of disparity and thus provides useful epidemiological input into decision making about policy to reduce cancer-related health disparities. This in no way precludes that there may be other valid inputs into the policy-making process that are based on different perspectives, such as a purely relative assessment of cancerrelated health disparities.

- To better monitor the population health burden of disparities over time, disparity indicators should be sensitive to two sources of change: change in the size of the population subgroups involved and change in the level of health within each subgroup. For instance, social policy can change both the number of people who are poor and the behavior and health status of the poor.

\section{Recommendations}

We recommend using a sequence of steps, described below, to assess health disparity. The first step is to inform any assessment of health disparity with a simple tabular and graphical examination of the underlying "raw" data (rate, proportion, etc., and subgroup population size). This may provide valuable insights into the basic question of whether the particular disparity has increased or decreased over time. The graphical presentation of the underlying data is depicted in Figure S2 (page 3), which shows educational disparity trends in the proportion of women not having had a mammogram for the past 2 years.

If, as for Healthy People 2010, the goal is to quantitatively monitor progress toward the elimination of health disparities across all social groups, then summary measures of health disparity are warranted. Figure S2 also contains two summary measures of health disparity-an absolute measure, the Absolute Concentration Index $(A C I)$, and a relative measure, the Relative Concentration Index $(R C I)$. The choice of specific summary measures also will be guided by whether the groups have an inherent ranking (such as education) or are unordered (such as gender).

Choosing measures of health disparity involves consideration of conceptual, ethical, and methodological issues. This report discusses some of these issues and provides recommendations for a suite of measures that can be used to monitor health disparities in cancer-related health outcomes.

Our recommendations for measuring disparity are:

1. To visually inspect tables and graphs of the underlying "raw" data. 
Figure S2. Proportion of Women Age 40 and Over Who Did Not Receive a Mammogram in the Past 2 Years by Level of Educational Achievement, 1990-2002, Trends in Absolute and Relative Disparity

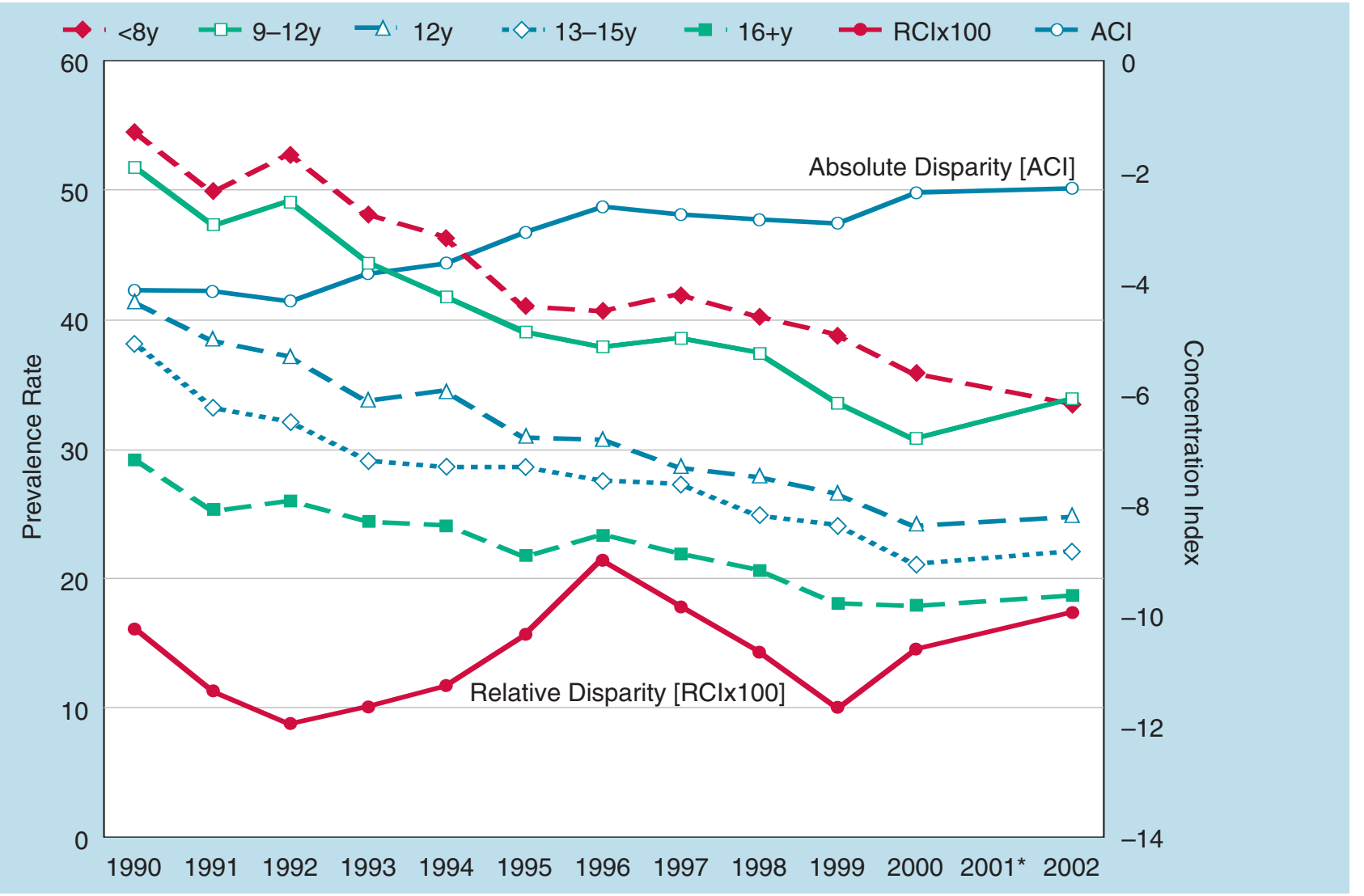

Source: CDC, Behavioral Risk Factor Surveillance Surveys 1990-2002.

*Note: Question not asked in 2001.

2. When the question involves only comparisons of specific groups, then pairwise absolute and relative comparisons may be sufficient. When the objective is to provide a summary across all groups, then the use of summary measures of health disparity is warranted.

3. If the social group has a natural ordering, as with education and income, then we recommend using either the Slope Index of Inequality (SII) or the Absolute Concentration Index $(A C I)$ as a measure of absolute health disparity, and either the Relative Index of Inequality (RII) or the Relative Concentration Index $(R C I)$ as a measure of relative disparity.

4. When comparisons across multiple groups that have no natural ordering (e.g., race/ethnicity) are needed, we recommend the Between-Group Variance $(B G V)$ as a summary of absolute disparity, and the general entropy class of measures, more specifically the Theil index and the Mean Log Deviation, as measures of relative disparity. 


\section{Introduction}

The goals of this report are to:

1. Highlight major issues that may affect the choice of disparity measure.

2. Systematically review measures of health disparity.

3. Provide a basis for selecting a "suite of indicators" to measure disparities in screening, risk factors, and other cancer-related health objectives.

\section{Initiatives to Eliminate Health Disparities}

In 1979, U.S. Surgeon General Julius B. Richmond first conceptualized the idea for national public health goals (3) and established specific public health objectives for reducing mortality and chronic illness in five age groups, which later were to be implemented in 15 strategic areas during the 1980s (4). Building on this foundation, Healthy People 2000 subsequently replaced the age-specific goals of 1990 with three overarching goals for the year 2000: increase the span of healthy life, reduce health disparities, and provide access to preventive health services (5). The explicit focus on reducing health disparities in Healthy People 2000 represented an important step toward establishing health disparities as a part of routine public health surveillance. Establishing different health targets for different social groups, however, could be construed as implying that a group's health potential was somehow constrained by its social-group membership, a factor over which group members may have little or no control. For example, the year 2000 target rate (per 100,000) for cancer mortality was 130 for the total population, but it was 175 for blacks.

The implication of setting different targets for different social groups was not lost on public health policy makers or politicians. In a 1998 radio address that celebrated Black History Month, President Clinton put forth a somewhat more radical national public health goal: "By the year 2010, we must eliminate racial and ethnic disparities in infant mortality, diabetes, cancer screening and management, heart disease, AIDS, and immunization." Racial and ethnic disparities in these and other areas are extensive and well documented, and given the context of their origins in the United States, there is ample reason to focus attention on their elimination. Similar health disparities, however, are evident not just between racial/ethnic groups but also between other social and demographic groups, a fact that now is reflected in the goals of Healthy People 2010 that specify eliminating health disparities by gender, income and education, disability, geographic location, and sexual orientation in addition to race and ethnicity (1). Similar health disparity targets also have been adopted by a number of state and local health agencies (see $6,7,8)$. The Healthy People 2010 policy goals thus 
represent an important shift toward "elimination," and not just "reduction," of existing health disparities.

The goal of eliminating health disparities also implies that a systematic scientific framework exists to measure health disparities and to monitor them over time across multiple social groups and measures of health status. We argue that no such clear-cut consensus framework currently exists in the United States, within either the research or the policy communities as to how health disparity should be measured. An important first step toward the elimination of health disparities is to carefully consider the conceptualization of health disparity to better understand what we mean by the term "health disparity," how we operationalize the concept of "eliminating health disparity," and how then to apply appropriate health disparity monitoring strategies.

\section{Cancer-Related Goals of Healthy People 2010}

The specific issues that motivate this project are related to the Healthy People 2010 framework for cancer-related goals, of which the overarching goal is to "reduce the number of new cancer cases as well as the illness, disability, and death caused by cancer" (9, page 3-3). The objectives for specific cancers are to reduce the rates of melanoma, lung, breast, cervical, colorectal, oropharyngeal, and prostate cancers, and, in keeping with the goals of Healthy People 2010, disparities in the above cancers and their major risk factors also should be eliminated. Thus, this report focuses on social-group and geographical disparity in cancer-related outcomes such as risk behaviors, screening, incidence, survival, and mortality.

Figure 1 (page 7) is typical of the sort of cancer-related data that motivate this project. These data show socioeconomic and racial/ethnic disparities in lung cancer mortality among U.S. females for 1995-1999. Although these data help to characterize disparity, they do not explicitly quantify the extent or variability in disparity. Several questions may be asked about this data. For instance, is the socioeconomic disparity in lung cancer mortality larger among Asian/Pacific Islanders or blacks? Or is the racial/ethnic disparity between non-Hispanic whites and blacks larger than the socioeconomic differences within each group? Additionally, variation exists in the direction of the socioeconomic disparity in different racial/ethnic groups. Among Hispanics, the age-adjusted death rate increases as area poverty decreases; among American Indian/Alaska Natives, however, rates increase as area poverty increases. Casual visual inspection of such graphs reveals that there are differences between and among groups. The challenge is whether we can move beyond the simple recognition of such differences (disparities) toward a strategy to quantify their magnitude in a scientifically reliable and transparent way that can be understood by all stakeholders. This will be even more important when monitoring changes in disparity over time.

Figure 2 (page 8) shows the annual rate of lung cancer incidence by race and gender for the period 1992-1999. How should we summarize the disparity in trends in lung cancer incidence? We might focus on comparing pairs of rates over 
Figure 1. Lung Cancer Mortality, Females, U.S., 1995-1999

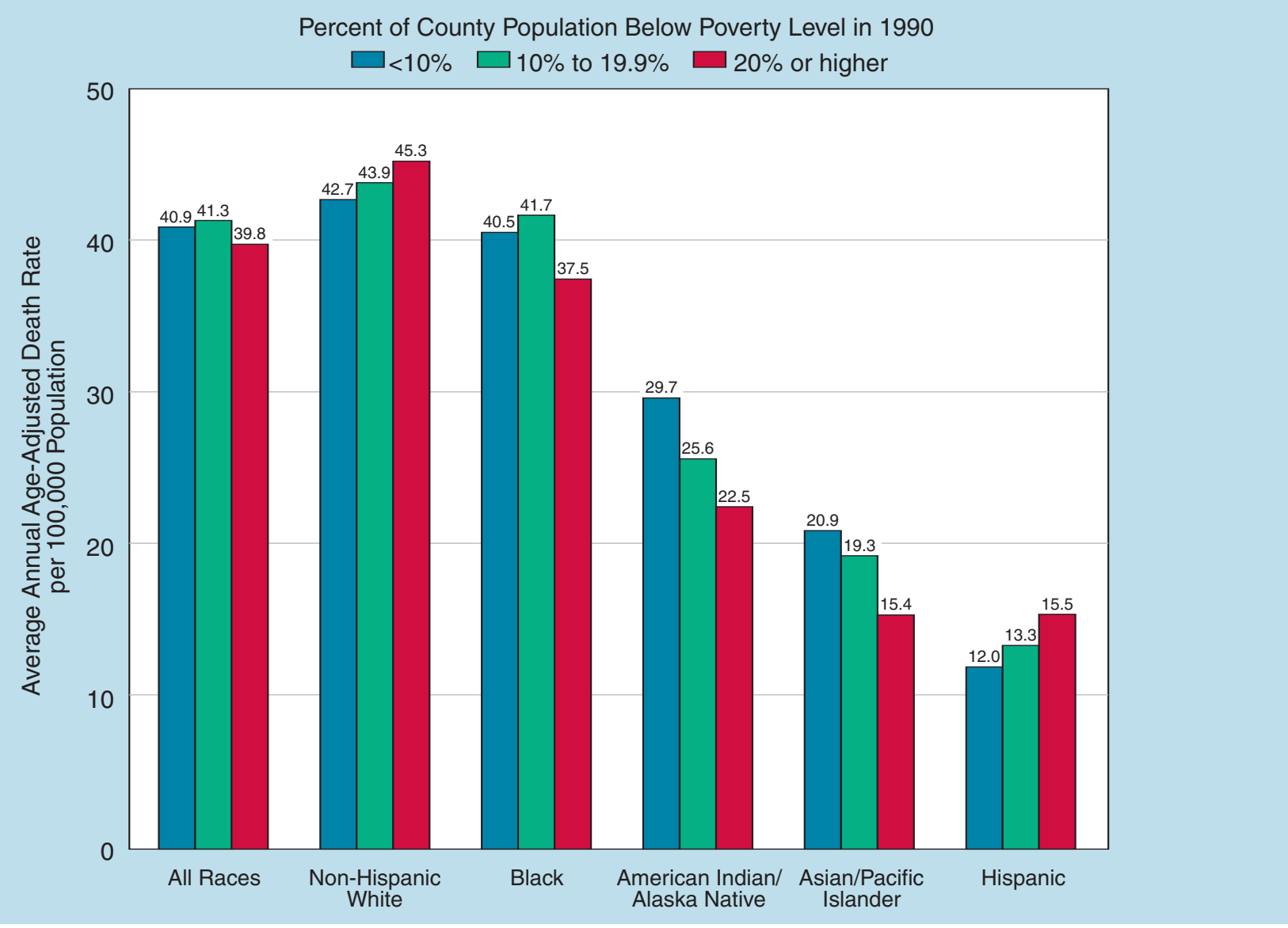

Source: Gopal Singh et al. Area Socioeconomic Variations in U.S. Cancer Incidence, Mortality, Stage, Treatment, and Survival, 1975-1999, 2003.

time-e.g., the gap between white and Asian/Pacific Islander females or between black males and black females. As the number of groups and years of data increase, however, there are diminishing returns to such a strategy because of the large number of possible pairwise comparisons and the inherent difficulty in summarizing them. For example, from Figure 2 in 1992, one could calculate the following incidence ratios: black to white males, 1.55; Asian/Pacific Islander to American Indian/Alaska Native males, 2.11; black to white females, 1.03; and Asian/Pacific Islander to American Indian/Alaska Native females, 1.71. The same comparisons in 1999 provide respective ratios of $1.48,2.29,1.12$, and 3.33. What can we conclude about the racial disparity in lung cancer incidence, given that incidence ratios are decreasing for some comparisons (e.g., black vs. white males) but increasing for others (e.g., Asian/Pacific Islander to American Indian/Alaska Native males)? There is no clear way to summarize the changes in these relative pairwise comparisons. Therefore, in addition to seeing how a particular social group's cancer-related health outcomes change with respect to another group, we also may be interested in whether we are making progress toward eliminating disparities across all racial/ethnic or socioeconomic groups, 
Figure 2. Lung Cancer Incidence by Gender and Race/Ethnicity, 1992-1999

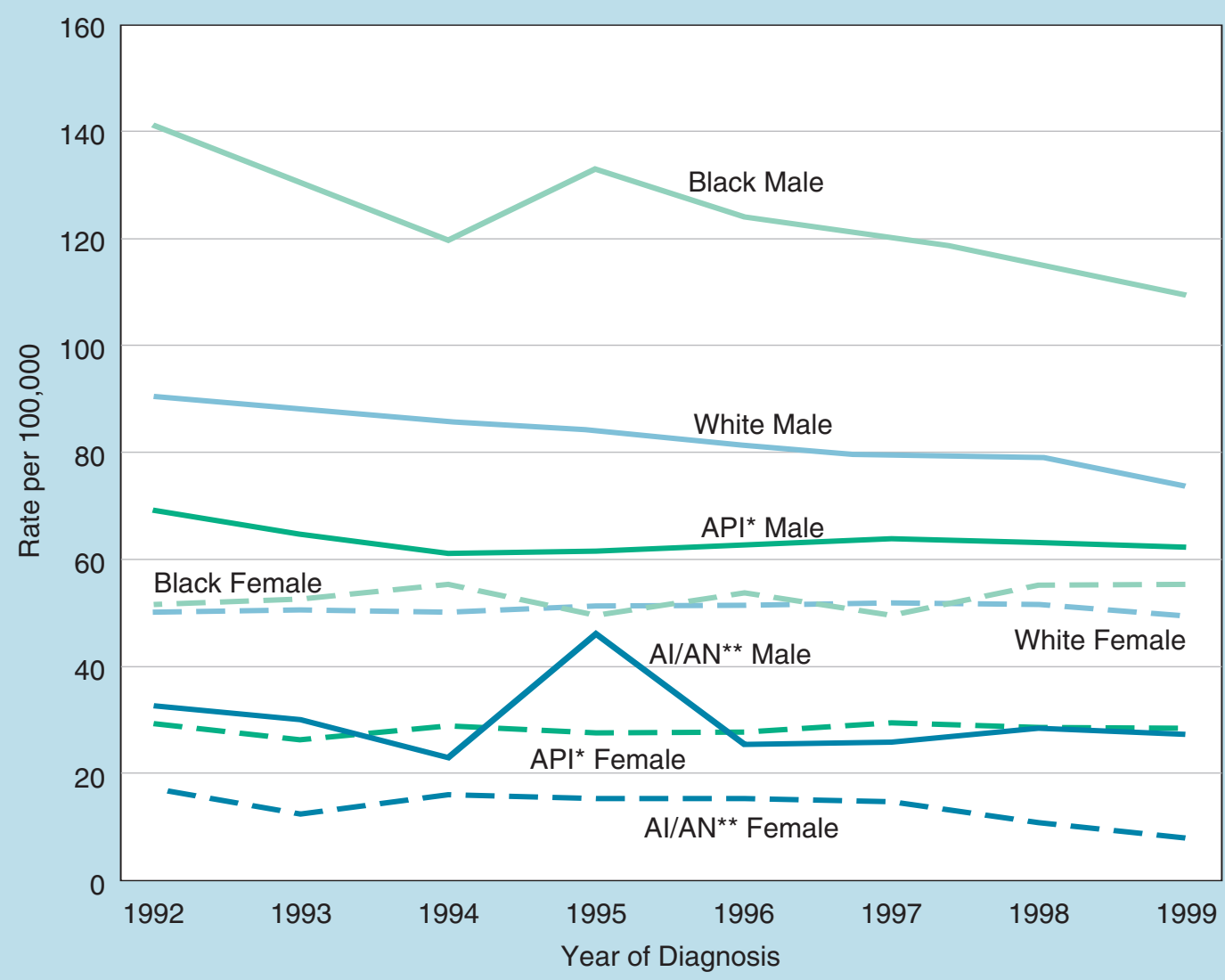

${ }^{*} \mathrm{API}=$ Asian/Pacific Islander

${ }^{* *} \mathrm{AI} / \mathrm{AN}=$ American Indian/Alaska Native

Source: Surveillance, Epidemiology, and End Results (SEER) Program (www.seer.cancer.gov) SEER*Stat Database: Incidence-Seer 11 Regs Public-Use, Nov. 2001 Sub for Expanded Races (1992-1999), National Cancer Institute, DCCPS, Surveillance Research Program, Cancer Statistics Branch.

which is consistent with the overarching goals of Healthy People 2010. That is, we may want to know whether the disparity in lung cancer incidence across all racial groups is decreasing. How should we answer that question when there are a multitude of pairwise and time-related comparisons that can be made? Pairwise comparisons have been the mainstay of epidemiological effect measures and clearly are central to disparity measurement, but there also is a place for summary measures of overall disparity.

\section{Brief History of Measuring Disparities in the United States}

\section{Measuring Disparities in Public Health}

This section briefly reviews selected historical studies of social-group disparities in health outcomes. Generally, the strong reliance in the past on pairwise relative and, less frequently, absolute disparity, and the difficulties such a 
strategy may raise for a broader, more populationfocused understanding of health disparities and their assessment over time, are emphasized. The task of measuring disparity in public health outcomes usually has been taken on by epidemiologists, who tend to rely on relative risk measures to characterize effect estimates (10). It is interesting that few references can be found to measuring health disparity per se in standard epidemiological texts. In some ways this is not surprising, but it helps to explain why standard epidemiologic metrics, such as relative and absolute risk differences, have been the general method of choice applied to measuring health disparity. A brief guide to health disparity measurement can be found in a recent textbook on epidemiologic methods in health policy (11), but this topic is not addressed in more recent foundational texts in either general epidemiology $(12,13)$ or social epidemiology $(14)$. This is not to suggest that more traditional epidemiologic measures are not applicable to the measurement and monitoring of health disparities, but that, before choosing the methods to best capture social concerns over the extent of health disparity and before attempting to devise policies to reduce/eliminate such disparities, one should be aware that such measures have certain limitations. These issues will be discussed in more detail later in this monograph.

\section{Trends in Social Group Health Disparities}

Are health disparities increasing in the United States? Despite consistent interest in social-group disparities in public health, limited data provide information on both social-group characteristics and health at the national and local levels (15-17). This in turn has resulted in a relatively small number of studies of health disparity trends for the United States as a whole. The landmark study in the social epidemiology of mortality by Kitagawa and Hauser (18), which involved a special matching of 1960 death-certificate records to the 1960 U.S. decennial census, serves as the benchmark against which most socioeconomic disparity trends are referenced. In that study, Kitagawa and Hauser measured disparity in terms of the standardized mortality ratio (SMR). The SMR is calculated as the ratio of the number of observed deaths to the number expected based on the mortality rates of the United States as a whole. If, for example, there is no educational disparity among white males ages $25-64$, then the number of observed deaths in each educational group should equal the number expected based on the mortality rate for all white males ages 25-64, corresponding to an SMR of 1.0. Kitagawa and Hauser found, however, that the SMR for white males ages 25-64 with less than 5 years of education was 1.15 (i.e., $15 \%$ more deaths were observed than were expected) and was 0.70 among those with a college degree (i.e., 30\% fewer deaths were observed than were expected). Generally, Kitagawa and Hauser found that higher socioeconomic position-whether measured by income or education -was associated with lower mortality and that mortality was higher among nonwhite and nonmarried individuals.

Interestingly, they also reported that education and income had independent effects-income disparities existed within education groups and educational disparities existed within income groups. It is important to note that, in terms of measuring disparity, this important study relied on pairwise comparisons of specific groups to the population average and did not use any summary measure of disparity. 


\section{Health Disparities According to Income}

Pappas and colleagues (19) used the National Mortality Followback Survey (NMFS) from 1986 to evaluate trends in education and income disparities since Kitagawa and Hauser's 1960 study. To use the information from all socioeconomic groups, Pappas and colleagues created a summary disparity measure. Similarly to Kitagawa and Hauser, they calculated an SMR for each socioeconomic group within gender and racial categories based on the sex-race-specific mortality rates for the entire United States. They then took the absolute value of the difference between each socioeconomic subgroup's (e.g., those with $<12$ years of education) $S M R$ and 1.0 and weighted it by the respective proportion of the population in that socioeconomic subgroup. Their index was the sum of these weighted absolute differences across all subgroups; thus, a value of 0.5 would be interpreted as the weighted average deviation of the socioeconomic groups' SMRs from 1.0. Pappas and colleagues found that mortality disparities had increased since 1960 for both whites and blacks, with steeper increases for income as compared with education as the measure of socioeconomic position. Thus, because the sum of population-weighted SMR differences for income increased more than for education, they concluded that income-related disparities increased more than educational disparities. Note that because each group's SMR was weighted by its population share, an increase in disparity when using this index could be observed even in the absence of changes in subgroup-specific mortality rates if the subgroups with the largest $S M R$ differentials increased their share of the population.
Duleep (20) used data linking the 1973 Current Population Survey (CPS) to Social Security longitudinal mortality data up to 1978 and also measured disparity by SMRs. Unlike Kitagawa and Hauser, however, she used her entire CPS sample-rather than the total U.S. population-to generate the expected number of deaths in each income group. She also concluded that socioeconomic disparities had not narrowed because the ratio of observed-to-expected deaths for most but not all income groups was further from 1.0 in 1973-1978 than it was in 1960. For example, the $S M R$ for individuals earning $\$ 10,000$ or more (the richest group) decreased from 0.84 in 1960 to 0.71 in 1973-1978. Schalick and colleagues (21), using the 1967 and 1986 NMFS, investigated disparity trends in mortality by income with different measures of disparity, the slope index and relative index of inequality. These disparity measures are similar to the index used by Pappas and colleagues (19) in that they weight each socioeconomic group by its population share, but the index is not based on SMRs. Rather, income groups are ordered from lowest to highest, and a line is fitted to the data using weighted linear regression. The slope of this line is the resulting "slope" index and is interpreted as the absolute difference in mortality across the entire range of income. Dividing this slope index by the actual mortality rate in the population gives the "relative" index and is the percent difference in mortality across the entire range of income. Similarly to Pappas and colleagues (19), Schalick and colleagues found that relative mortality disparities increased when measured by the relative index of inequality, particularly for males; they also found that absolute disparities decreased during the same period when measured by the 
slope index of inequality, primarily because the absolute declines in mortality were greater for the least well-off groups.

Finally, using a different measure of disparity, the Population Attributable Risk percent (PAR\%), Hahn and colleagues reported that the share of mortality in the United States due to poverty had increased from 1973 to 1991 (22). The PAR\% essentially is a summary index designed to estimate the population health impact of eliminating health-damaging exposures and is a function of the prevalence of the exposure and its associated relative risk. In this case the exposure is poverty, and the interpretation of the index is the percent by which the population death rate would decrease if poverty were eliminated. Thus, the $P A R \%$ is a population-focused disparity index in that it measures the impact on the total population of eliminating the health disparity between the poor and the nonpoor. If the poor represent a small fraction of the population, or if the health effects of being poor are small, then the $P A R \%$ will show that the elimination of the exposure-poverty-will have a marginal effect on population health. Hahn et al. report that, from 1973 to 1991, the PAR\% increased from $16.1 \%$ to $17.7 \%$, indicating that the population health benefit of eliminating mortality disparities by poverty status increased. The increase, however, was due entirely to an increased $P A R \%$ among men, as the $P A R \%$ decreased for both black and white women.

\section{Health Disparities According to Education}

Feldman and colleagues (23) investigated trends in educational disparities in mortality among whites between 1960 and 1971-1984 using the matched data of Kitagawa and Hauser and the first National Health and Nutrition Examination Survey Epidemiologic Followup Study (NHEFS). They measured disparity using a standard epidemiological "rate ratio" - the mortality rate in the least-educated group divided by the mortality rate in the most-educated group (i.e., a pairwise comparison of extreme socioeconomic groups). The researchers concluded that educational disparities increased, but this effect was primarily seen among white men. Interestingly, in their discussion, Feldman and colleagues noted that the distribution of education changed enormously over the period of study but concluded that the magnitude of the increase was "probably not large enough to have a major impact on trends in differentials" (23, page 929). The researchers, however, did not empirically examine this assumption, which perhaps is why Elo and Preston revisited this question using the same data (24) and conducted a similar analysis of trends in educational disparity in mortality using multiple measures of disparity (slope index of inequality, relative index of inequality) that account specifically for the changing distribution of education over time. Similar to previous analyses $(19,23)$, Elo and Preston found that the educational disparity had increased among white men. Whereas Feldman and colleagues found no change or a small disparity increase for white women, however, Elo and Preston found that both absolute and relative disparities had decreased for white women of all ages. These studies highlight the important issue of whether measures of health disparity should be sensitive to changes in the size of the "exposed group"-in this case, the most disadvantaged in terms of income or education. The issue of the effect on health disparities of the movement of individuals 
into and out of different social groups over time also is important and has been neglected somewhat in the United States, despite having received consistent emphasis in the health disparities literature (25-27).

The above studies indicate that relative mortality disparities generally appear to have increased since 1960, but the extent of disparity differs with different measures of disparity and socioeconomic position. Because all of the above analyses use different data sources and different measures of health disparity, it is difficult to reach a firm conclusion as to how much the socioeconomic disparity in overall mortality has increased or decreased over time. This perhaps is not surprising given that, even for simple disparity measures such as the relative comparison of the lowest and highest social groups, different national data sources can provide different estimates of the size of the same health disparity (28).

\section{Health Disparities According to Race/Ethnicity}

Despite the longstanding interest in health disparities between racial/ethnic groups in the United States, surprisingly few studies have analyzed racial/ethnic disparity trends. Additionally, the major racial/ethnic focus in the United States has been on disparities between blacks and whites (or nonwhites and whites), which makes understanding trends somewhat less difficult because the inequality between two groups may be summarized easily with either a simple difference or ratio measure. The continuing increase in U.S. racial/ethnic diversity and the growing need to compare multiple racial/ethnic groups and to examine individual populations that usually are grouped together (i.e., Chinese with Japanese or Mexican Americans with Puerto Ricans), however, make the use of pairwise comparisons for summarizing inequality trends more difficult to understand and communicate. The inherent difficulty of talking about trends in health inequality by reference to several relative risks is one reason for attempting to summarize inequality with a single index. One potential summary measure, the Index of Disparity $\left(I D_{\text {isp }}\right)$, was introduced formally by Pearcy and Keppel (30) and was applied to 17 health status indicators during the period 1990-1998 for five racial/ethnic groups: nonHispanic whites, non-Hispanic blacks, Hispanics, American Indian/Alaska Natives, and Asian/Pacific Islanders (31). The $I D_{\text {isp }}$ measures variations in health across dimensions of a social group (e.g., race/ethnicity) relative to some reference pointin this case, the total population rate. Thus, a decline in the $I D_{\text {isp }}$ indicates that the variation in health across racial/ethnic groups declined relative to the total population rate. From 1990 to 1998, the researchers found that the $I D_{\text {isp }}$ decreased for most mortality measures and infant health outcomes (i.e., racial/ethnic disparity decreased), but increased for teenage pregnancy, motor vehicle deaths, suicide, work-related injury deaths, and tuberculosis case rates. It is important to note that, unlike some disparity measures mentioned previously, the $I D_{\text {isp }}$ does not weight social groups by their population share. That is, the $I D_{\text {isp }}$ takes a perspective on disparity that what matters is the difference in subgroup rates of health, regardless of the number of individuals that may be affected. Thus, it is more focused on strict equality of health status measures, regardless of social-group size and the extent to which social-group health differences may impact population health. 


\section{Socioeconomic Disparity Trends in Cancer}

In general, there have been fewer studies of socioeconomic disparity trends in cancer incidence and mortality. One of the difficulties in monitoring disparity trends in cancer with respect to socioeconomic groups is that the major source of data on cancer incidence and survival, the National Cancer Institute's Surveillance, Epidemiology, and End Results (SEER) Program, does not collect socioeconomic data on individuals (17). A number of studies, such as those by Singh and colleagues (32) and Krieger and colleagues (33), however, have used information on residential location collected on incident cancer cases to create a measure of socioeconomic position. This is accomplished by linking the neighborhood or county in which an individual cancer case resides to the U.S. census to get a measure of the socioeconomic status of that area-for example, the poverty rate. Such "areabased" measures of socioeconomic position certainly are an improvement over having no measure at all, but they also require additional assumptions that may hinder their utility for monitoring cancer-related disparities. For example, the use of area-based measures assumes that the average socioeconomic status of the area is representative of the status of the individual, and that, because the census is conducted only every 10 years, the socioeconomic status of an area in, say, 1990 is an accurate representation of the same area for a cancer case diagnosed in 1997.

Using area-based measures of socioeconomic position (e.g., census tract poverty rates), Singh reported a reversal in the socioeconomic gradient among men in overall cancer mortality from 1950 to 1998 (34). Singh used relative pairwise comparisons of the highest and lowest socioeconomic groups and showed that, in 1950, mortality rates were $49 \%$ higher in higher socioeconomic areas; this disparity decreased over the next 30 years and, by the late 1980s, cancer mortality rates were 19\% higher in lower socioeconomic areas. Thus, over the past 50 years, the pattern of higher cancer mortality among individuals in higher socioeconomic areas disappeared and was replaced by a pattern of higher cancer mortality among individuals of lower socioeconomic position. A similar pattern of reversing gradients also was evident for lung cancer and colorectal cancers (35). With regard to cancer incidence, from 1975 to 1999, the trend in socioeconomic disparity for all cancers among both men and women was inconsistent (32) as measured by the incidence rate among those living in areas with $>20 \%$ of the population in poverty relative to the rate in areas with $<10 \%$ in poverty (i.e., relative pairwise comparison of extreme groups). This likely is due to differing disparity trends for specific cancer sites. Compared to the highest socioeconomic group, cancer mortality rates were higher among the lowest socioeconomic group for lung and prostate cancers among males, and the ratio of the lowest to the highest socioeconomic area widened from 1975 to 1999. Incidence of melanoma was higher among males in higher socioeconomic areas in 1975, and this relative difference increased by 1999. Colorectal cancer was more frequent among males in higher socioeconomic areas in 1975; the relative difference decreased by 1999 . Among females, women in poorer socioeconomic areas had higher incidences of lung and cervical cancers in 1975; the disparity in lung cancer incidence 
remained relatively constant, whereas the disparity for cervical cancer declined. Women living in higher socioeconomic areas had a higher incidence of melanoma, colorectal, and breast cancers in 1975; by 1999, this disparity narrowed for colorectal cancer and widened for breast cancer and melanoma. This analysis highlights the importance of examining site-specific rather than overall cancer trends, as the overall cancer rate is a diverse amalgam of specific types of cancer that differ in their etiology and, therefore, their social distribution.

Few studies have assessed trends in educational disparities in cancer. Steenland and colleagues (36) analyzed trends in educational disparities in cancer mortality using data from the American Cancer Society's Cancer Prevention Study cohorts (CPS-I and CPS-II). They used ordinary least squares regression to calculate a regression-based relative effect of education. Instead of simply comparing the most- and leasteducated groups, this disparity measure uses the mortality rates for all educational groups and is interpreted as the increase in cancer mortality for each 1-year decrease in the number of years of education. The study found that educational disparities increased from 1959-1972 to 1982-1996 for lung and colorectal cancers and decreased for breast cancer. The researchers did not, however, account for changes in the social distribution of education during this period and were forced to conclude that "the educational categories were not comparable between the two populations." (36, page 20 ). Thus, their conclusions were less than clear. If the education categories are not comparable, and this fact is not accounted for in the disparity measure, then it is difficult to know how to interpret the reported disparity trend among these cohorts.

\section{Racial/Ethnic Disparity Trends in Cancer}

Although racial/ethnic disparities in cancer have received significant attention, especially with regard to treatment $(2,37)$, relatively few studies have assessed long-term trends in these disparities. Again, the general lack of detailed historical racial/ethnic information in cancer-related data sources often limits analyses of long-term disparity trends to a pairwise comparison of whites and blacks or whites and nonwhites. Within the last decade, focus on and efforts to promote population health data for major ethnic groups have increased. Ten-year trends now are available for some groups. For subgroups within major racial/ethnic groups, this is complicated further by the lack of inter-censal estimates of population size as well as issues of comparability of reporting for numerator (incidence) and denominator (population size) data. Other issues that arise in comparing groups by race/ethnicity include differences between subpopulations commonly grouped together, such as differences in cancer incidence rates between American Indians and Alaska Natives and between various American Indian tribes.

With regard to mortality from all cancers, whites had higher mortality rates than nonwhites until the middle of the $20^{\text {th }}$ century, after which nonwhites have had higher mortality rates. The gap between whites and nonwhites increased from the mid- $20^{\text {th }}$ century until the early 1990 s, after which it declined $(38,39)$. The primary reasons for the widening gap between white and 
nonwhite cancer mortality since mid-century were relatively larger increases in nonwhite mortality from lung, prostate, colorectal, breast, and ovarian cancers (39).

Still fewer studies have attempted to use any summary measure of health disparity across several racial/ethnic groups. Keppel and colleagues used the Index of Disparity to compare lung and female breast cancer mortality rates in 1990 and 1998 across five racial/ethnic groups: nonHispanic whites, non-Hispanic blacks, Hispanics, American Indian/Alaska Natives, and Asian/Pacific Islanders (31). Racial/ethnic disparity declined for both cancers-significantly so for lung cancer.

\section{Health Inequality and Health Inequity}

The language of "eliminating health disparities" seems simple and straightforward-something that everyone understands in the same way and can agree on. When we say we want to eliminate health disparity, do we really mean we want everyone to have the same level of health? Is the goal that all individuals/social groups should have the same health, regardless of how healthy or sick they might be? Or do we mean that it is improving the health of the most disadvantaged individuals/social groups so that they approach the health of the more advantaged (i.e., priority to the worst-off/least healthy)? In regard to reducing income disparities, we are comfortable as a society in considering the need to reduce the incomes of the advantaged via taxation in order to increase the incomes of the impoverished. In other words, we are willing to engage in policy discussions focused on income redistribution from the rich to the poor. It is not clear that this idea applies to health disparity. That is to say, in public health we generally are not willing to accept health declines in a healthier or more socially advantaged group to foster improved health in those who are less healthy or socially disadvantaged. Yet, it is plausible that, for example, the health of the rich and the poor both improve, but the rich improve at a better rate, therefore increasing the relative disparity between the two groups. This situation highlights the possible tension that may arise in designing policies to simultaneously achieve the two overarching goals of Healthy People 2010 improving average health and eliminating health disparities. Such questions only scratch the surface but underscore the potential implications of a literal interpretation of the language of the Healthy People 2010 initiative to "eliminate" health disparities (1).

The health disparity concept involves both descriptive and normative elements. The task is to understand what the elements are and to develop sensible measures of disparity that capture both of these dimensions $(40,41)$. In the United States the use of the term "disparity" implies two core concepts. First, it suggests that there are health "differences" between individuals or social groups; second, it suggests that such differences in some way are unfair and an affront to our moral concepts about social justice. Thus, the term "disparity" often mixes ideas of "inequality" and "inequity." The term "inequality" literally means difference-that two quantities are not the samebut the term "inequity" implies an ethical judgment about those differences. Inequality is a measurable, observable quantity that can be reasonably and unambiguously judged; inequity relies on a moral, ethical judgment about justice 
and thus is not unambiguously measurable or observable. The classification of health differences as unequal is a relatively easy task compared to the classification of health differences as inequitable. Judgements concerning inequity rely on social, political, and ethical discourse about what a society believes is unfair (42).

Another crucial dimension to ideas of inequity and concepts of justice comes from discussions about disparities in health that are avoidable and those that are unavoidable $(43,44)$. Both types contribute to health disparities, but only potentially avoidable determinants contribute to inequity (45). Thus, "avoidability" implies a capacity to intervene (via social policy, medical care, etc.) with respect to the determinants of disparity. It often is difficult to identify the determinants of disparities or to distinguish between avoidable and unavoidable determinants. Determinants of disparity may be unavoidable in the short run and avoidable in the long run. It is easier to measure disparity between groups than it is to identify the determinants of the disparity or to decide which determinants are avoidable and which are unavoidable. To eliminate disparities in health between groups, however, the determinants of disparities in health must be identified and avoidable determinants modified. The first task, though, is to arrive at methods to identify and quantify health disparities over time as the basis for evaluation and action. 


\section{Defining Health Disparities}

This section introduces concepts of health disparity and discusses important issues involved in their measurement. It also highlights the fact that "disparity" is a fundamentally ambiguous concept with multiple dimensions that different measures of disparity emphasize to a greater or lesser extent. On its face, the concept of a health disparity seems rather simple. In fact, when one attempts to formally define what constitutes a health disparity, difficulties emerge. For example, consider the following definitions of what constitutes a health disparity for the purposes of measurement:

"Health disparities occur when one group of people has a higher incidence or mortality rate than another, or when survival rates are less for one group than another."-NCI Center to Reduce Cancer Health Disparities, 2003 (46)

"A population is a health disparity population if...there is a significant disparity in the overall rate of disease incidence, prevalence, morbidity, mortality, or survival rates in the population as compared to the health status of the general population."-Minority Health and Health Disparities Research and Education Act of 2000 (47, page 2498)

"For all the medical breakthroughs we have seen in the past century, there remain significant disparities in the medical conditions of racial groups in this country.... [W] hat we have done through this initiative is to make a commitment-really, for the first time in the history of our government-to eliminate, not just reduce, some of the health disparities between majority and minority populations."-Dr. David Satcher, Former U.S. Surgeon General, 1999 (48, page 18-19)

"Health disparities are differences in the incidence, prevalence, mortality, and burden of diseases and other adverse health conditions that exist among specific population groups in the United States." -NIH Strategic Research Plan and Budget to Reduce and Ultimately Eliminate Health Disparities, Vol. 1, Fiscal Years 2002-2006

Although these definitions share the same basic sentiment, there are some potentially important differences that reflect underlying assumptions (explicit or implicit) about what constitutes a health disparity. For instance, under the first definition above, a disparity is a difference in health between any two populations, whereas in the second definition (from the law that established the NIH initiative), a disparity is a difference in health between some specific population and the general population. This definition also introduces the idea that a disparity must be "significant" in magnitude. These differences may seem to be inconsequential semantics, but for the purposes of monitoring 
progress toward eliminating health disparities, the different definitions imply different metrics for assessing progress. One could imagine a scenario in which two minority groups have identical mortality rates, both of which differ substantially from that of the general population. A more extreme (but unlikely) scenario might be a case in which one minority group's health is better but not "significantly" different from that of the general population, whereas another minority group's health is worse but also not "significantly" different from that of the general population. It is possible, however, that the difference in health between the two minority groups is "significant." Thus, for the same observed data we might conclude either that a disparity exists (under the first definition above) or that a significant disparity does not exist (under the second definition above). Also note that the definition offered by former Surgeon General Satcher states that disparity exists between the minority and majority population, which suggests a third possible reference point-the majority population-though it is not clear how that majority is to be defined.

Our purpose is not to focus on semantics but rather to illustrate the lack of clarity in health disparity definitions and how this is important in choosing measures to monitor disparity. It is unlikely we will agree on a single definition of disparity. It is more likely that there are several legitimate, competing perspectives on health disparity that can be adopted. We want to emphasize the importance of understanding the link between ethical perspectives and the choice of quantitative health disparity measures. 


\section{Issues in Evaluating Measures of Health Disparity}

This section discusses several issues-conceptual, pragmatic, and technical —-that potentially are important in choosing health disparity measures. Many of these issues receive expanded discussions in the more technical descriptions of the measures that follow in later sections. The intention here is to highlight the set of main issues that might be considered.

\section{Total Disparity vs. Social-Group Disparity}

There is an important conceptual issue regarding the specific quantity to be determined when evaluating health disparities. The fundamental distinction to be made is between measuring total disparity, or total variation, and measuring disparities between social groups. The former involves evaluating the univariate distribution of health among all individuals in a population, without regard to their group membership; the latter involves assessing health differences between individuals from certain a priori chosen social groups. The World Health Organization (WHO) initiative to measure health inequality, led by Chris Murray and colleagues, has advocated strongly for an approach to the measurement of health disparity as total health disparity among individuals that is blind to social groups $(49,50)$. Initially, this seems at odds with our notions of why we are evaluating disparity in the first place (51). That is, the initiative to eliminate health disparities arose within the United States because of the persistent presence of social-group health disparities, not out of concern for a widening overall distribution of health. Yet, a deeper understanding of the overall task of determining variation in population health requires that we appreciate the concept of total health disparity. It is likely that the between-group disparity we seek to measure in regard to initiatives such as those in the United States may be relatively small compared to the total disparity that exists between individuals in a population.

Figure 3 (page 20) shows the average body mass index (BMI) for five education groups in the 1997 National Health Interview Survey (NHIS). It is clear that there is a gradient of decreasing BMI with increasing education when comparing average BMI among education groups. The plots of the $10^{\text {th }}$ through the $90^{\text {th }}$ percentiles of BMI, however, show that there is much greater variation in BMI within education groups than between education groups. Thus, basing the measure of health disparity on between-group average differences may not capture much of the total health variation among individuals. This is not a problematic statement itself but should be understood-and is why indicators of total health inequality can be informative. Thus, based on the group averages and a desire to reduce obesity in the population, focusing a health intervention on the "high-risk" social group (those with less than an $8^{\text {th }}$-grade education) will in practice target only a limited proportion of those at high risk, because 
Figure 3. Mean and 10th-90th Percentiles of Body Mass Index by Education, NHIS, 1997

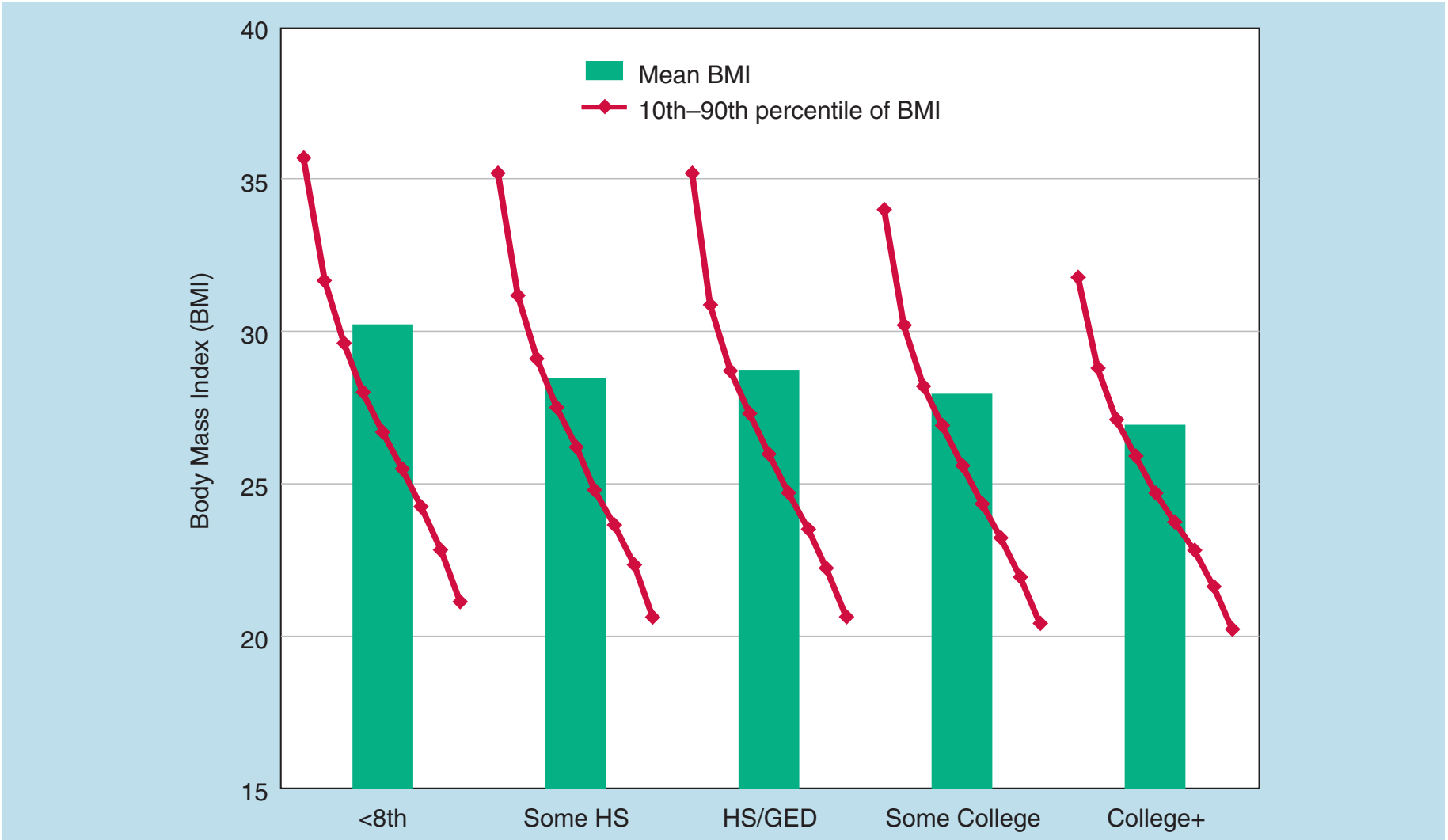

high-risk individuals exist in every education group.

Measures of total health disparity may mask substantial social-group disparities, however. Figure 4 (page 21), adapted from Asada and Hedemann (52), shows the population distributions of life expectancy in two hypothetical societies, A and B. Both populations have the same average life expectancy, but Society A has a much narrower overall distribution of life expectancy; were we to use a measure of total disparity, we would judge Society A to have the smaller disparity. Within Society A, however, there is a substantial gap in life expectancy between social groups 1 and 2, whereas in Society B, groups 1 and 2 have nearly identical life expectancy distributions. If we use a measure of social-group disparity, we likely would judge Society A as having the greater disparity because the distribution of life expectancy between the groups is unequal. The point of this example is to show that measures of total disparity and measures of group disparity may or may not lead to similar judgments about the extent of disparity in two populations or at two time periods. Thus far, the evidence seems to indicate that total disparity and social-group disparity measure different aspects of population health. Two crossnational studies found little correspondence between measures of total disparity and measures of socioeconomic disparity for either child (53) or adult (54) mortality. That is, countries with the largest amount of overall mortality variation did 
not necessarily have larger socioeconomic mortality variation, and countries with the largest socioeconomic mortality disparities did not have the largest overall mortality disparities.

\section{Relative and Absolute Disparities}

The most frequent method of communicating information about social disparities in public health and epidemiology is in relative termsthrough measures of association such as the relative risk. In epidemiology, relative risks are the most common measures of "effect size," partly because they have advantageous properties not shared by absolute risk differences $(12,55)$. Relative and absolute health differences between social groups are the primary language of health disparities, but they provide fundamentally different types of information. Figure 5 (page 22) demonstrates this essential point by showing trends in absolute and relative disparity between males and females in stomach cancer mortality over the past 70 years. Clearly, there was enormous progress in reducing stomach cancer mortality rates among both males and females during the $20^{\text {th }}$ century. As the rates for both groups declined, however, the ratio of male-tofemale mortality (i.e., the relative disparity)

\section{Figure 4. Hypothetical Distributions of Life Expectancy in Two Populations}

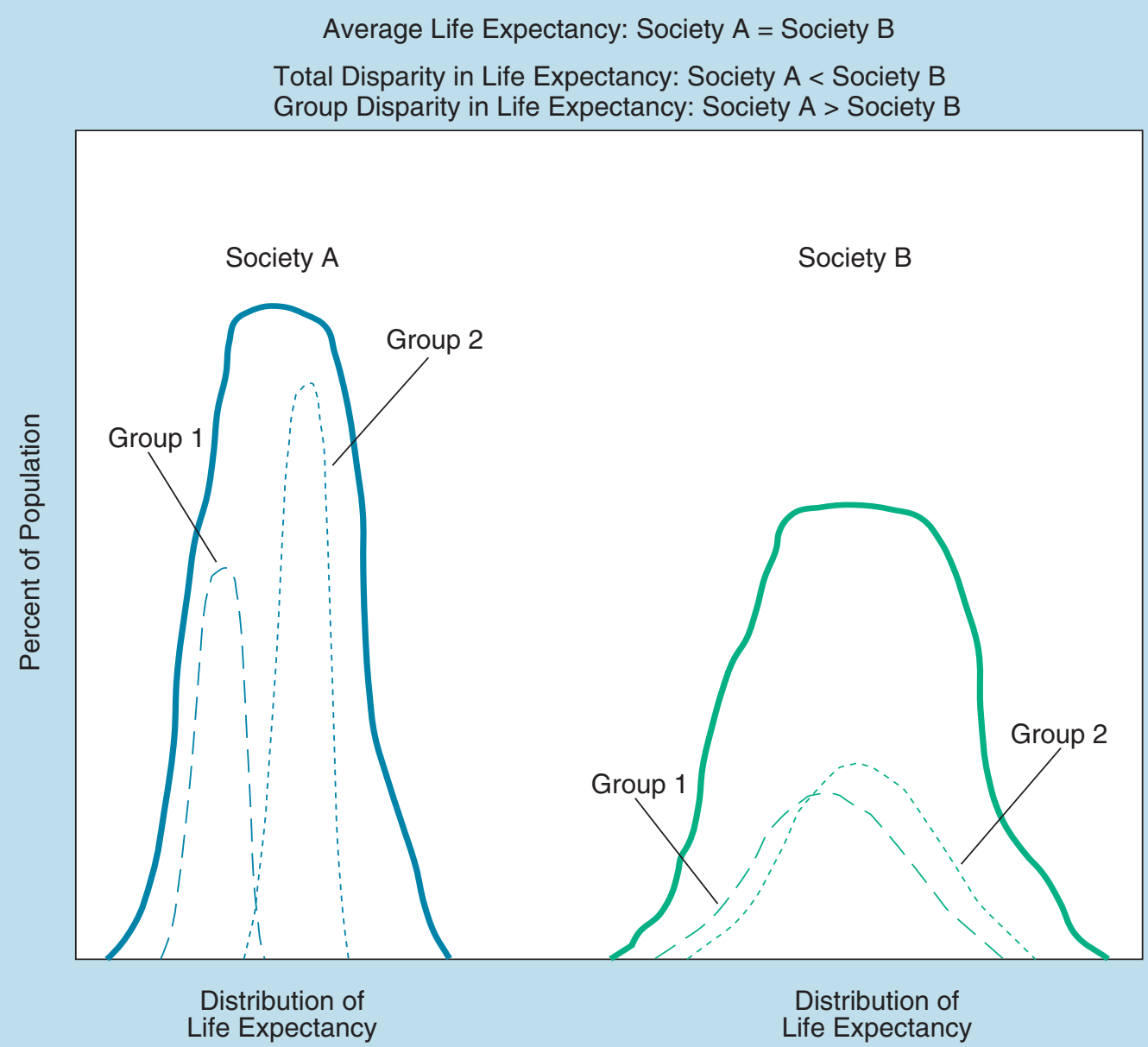


steadily increased. If the difference between male and female mortality (i.e., the absolute disparity) is used as the measure of disparity, however, we observe a different trend. The male-female gap increased from 1930 to about 1950, as female rates declined faster than male rates, and has declined steadily since 1950. Thus, Figure 5 illustrates the possibility that one might arrive at opposite conclusions about what happened to this health disparity, depending on which measure was chosen-the absolute or relative disparity. The reason is that the relative disparity cannot reflect changes in absolute rates-the disparity is relative to the rate in the comparison group.

\section{Reference Groups}

The language of disparity—defined literally as "difference"-implies a comparison group. A major question in choosing disparity measures is the choice of comparison group. As noted above, the different definitions of disparity imply different comparison groups, and thus the answer one would get about the extent and patterning of disparity may differ according to which groups are compared. Figure 6 (page 23) shows the situation for cervical cancer mortality rates among several racial/ethnic groups. Hispanic women clearly have the highest incidence of cervical cancer, but how

\section{Figure 5. Absolute and Relative Gender Disparity in Stomach Cancer Mortality, 1930-2000}

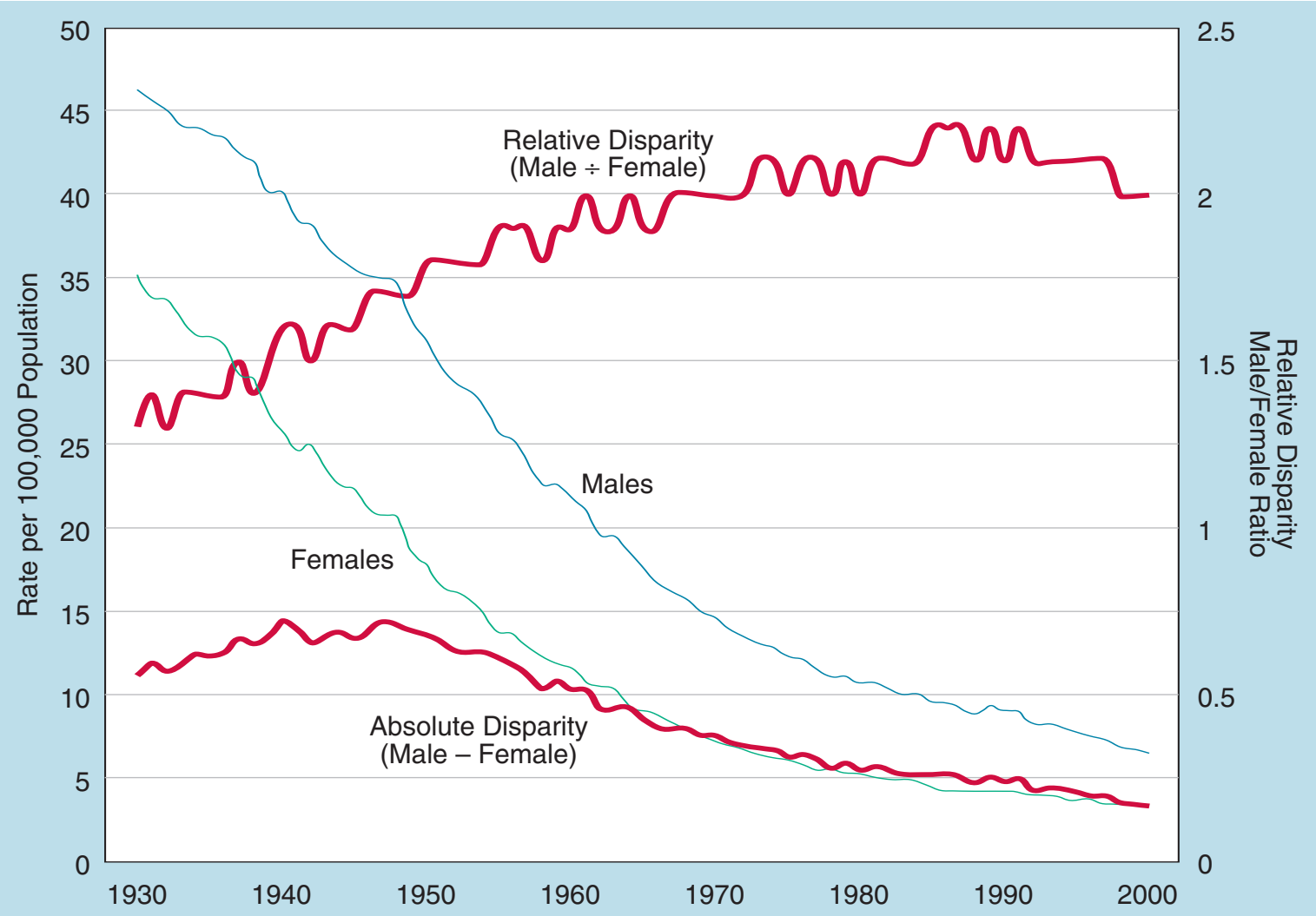

Source: Wingo et al. Cancer 2003;97(11 Suppl):3133-275, and SEER Cancer Statistics Review, 1975-2000. 
large is the disparity in cervical cancer incidence? The answer depends on the choice of the reference group. If the Hispanic disparity is measured relative to the general population (i.e., the total rate), then the relative disparity is 1.75 . If, however, we follow Dr. Satcher's recommendation (48) and focus on the disparity from the majority population-non-Hispanic whitesthe relative disparity is 2.21 . Or, if the "best-off" group-American Indian/Alaska Natives_-is chosen as the reference group, we obtain a relative disparity of 2.43 .

\section{Average Population Member}

One logical reference group might be the population average, where the disparity measure reflects the gap between the health of different social groups and the mean health of the entire population. The population average is appealing intuitively as a reference point and, as noted above, often is also used explicitly in defining what constitutes a health disparity.

Figure 6. Relative Risk (RR) of Incident Cervical Cancer Among Hispanics According to Varying Reference Groups, 1996-2000

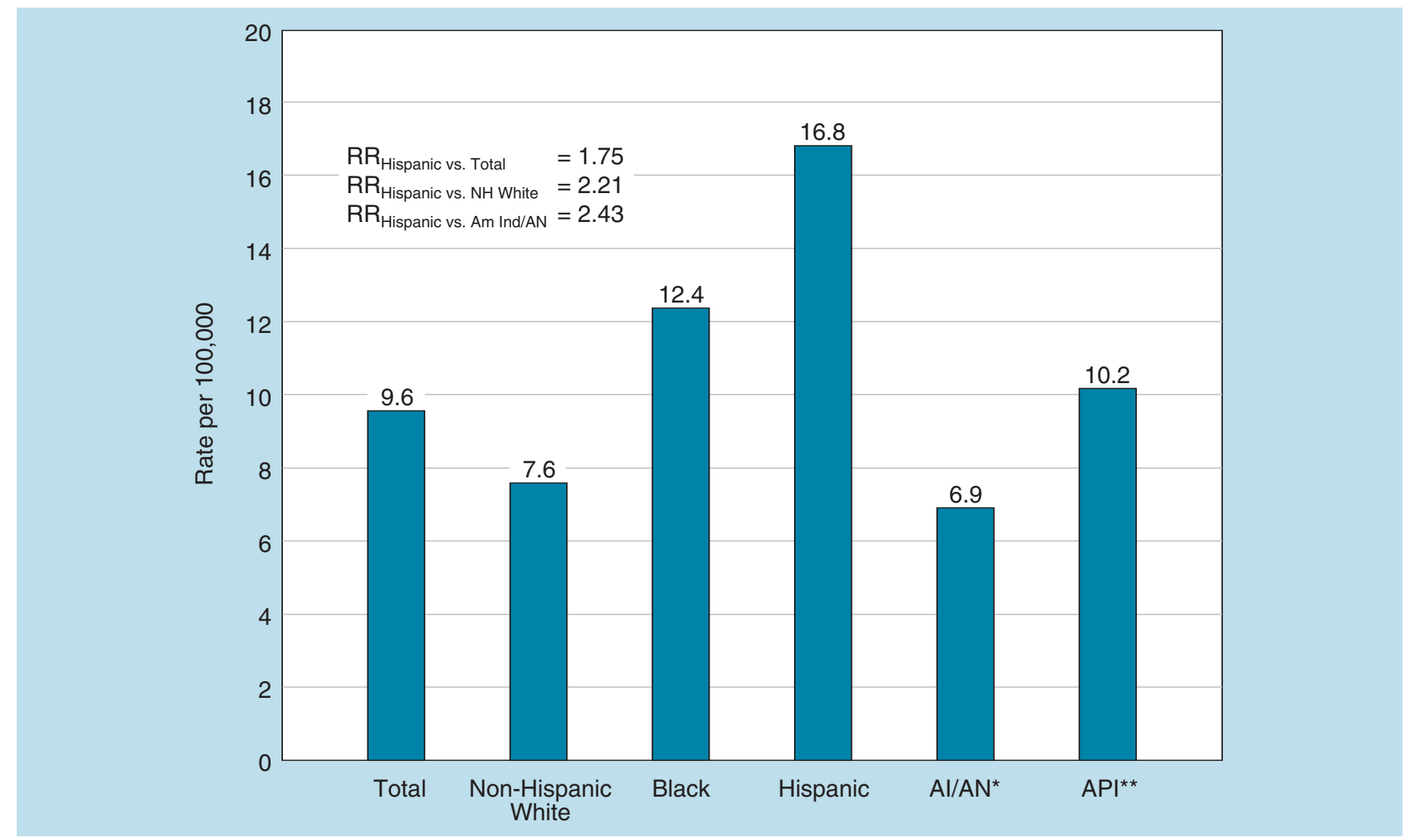

*AI/AN = American Indian/Alaska Native

**API = Asian/Pacific Islander

Source: SEER Cancer Statistics Review, 1975-2000. 


\section{Best-Off Group/Person/Rate}

This perspective suggests that one might measure disparity as a difference between each social group compared to the healthiest group (or even the healthiest person). This is similar to Sen's concept of shortfalls (56), in which it is assumed implicitly that every social group in the society has the potential to achieve the health of the best-off group. It should be noted, however, that the bestoff social group may be relatively small in size, which may lead to substantial variation and instability and could make assessing trends in disparities more difficult.

\section{All Those Better Off}

It also is possible to measure disparities by comparison to all those individuals or groups that are better off than a particular group or person. This may seem similar to the "best-off group" reference point, but it differs in a subtle way that may best be illustrated with an example using actual cancer data. Figure 7 (page 25) shows cancer incidence from 1996-2000 by race and ethnicity for two different cancers, kidney/renal pelvis and myeloma. In both cases, there is a substantial difference between the group with the highest incidence rate, blacks, and the group with the lowest or "best" rate, Asian/Pacific Islanders. When we look at the incidence rates of other groups, however, we see two different situations.

In the case of kidney cancer, Hispanics and whites have rates more similar to blacks, whereas, in the case of myeloma, they have rates more similar to Asian/Pacific Islanders. Relative to all those better off than blacks, most people might judge the disparity to be worse in the case of myeloma compared to kidney cancer; yet, if measured relative to the "best-off" group perspective, we would be unable to capture this nuance.

\section{Fixed/Target Rate}

The prior three reference groups are inherently relative as they change over time, which may make assessments of trends in disparities inconclusive if using pairwise comparisons. One advantage of a fixed or target rate is that the reference level does not change over time unless a new target is adopted.

\section{Social Groups and "Natural" Ordering}

The Healthy People 2010 initiative mandates eliminating health disparities within a number of different types of social groupings: gender, income and education, disability, geographic location, sexual orientation, and race and ethnicity. Such groupings were chosen because they represent important normative dimensions of U.S. society, and it has been shown repeatedly that health differences exist between these social groups. The above groups, however, also differ in ways that may have implications for monitoring health disparities. The social groups that measure dimensions of socioeconomic position-education and income-have an inherent ordering regardless of the health status of their members. Individuals with less than a high-school education unambiguously have less formal education than do individuals with a college degree. The same cannot be said for the other groups targeted by the Healthy People 2010 initiative. There simply is 
Figure 7. Age-Adjusted Incidence of Kidney/Renal Pelvis Cancer and Myeloma by Race and Ethnicity, 1996-2000

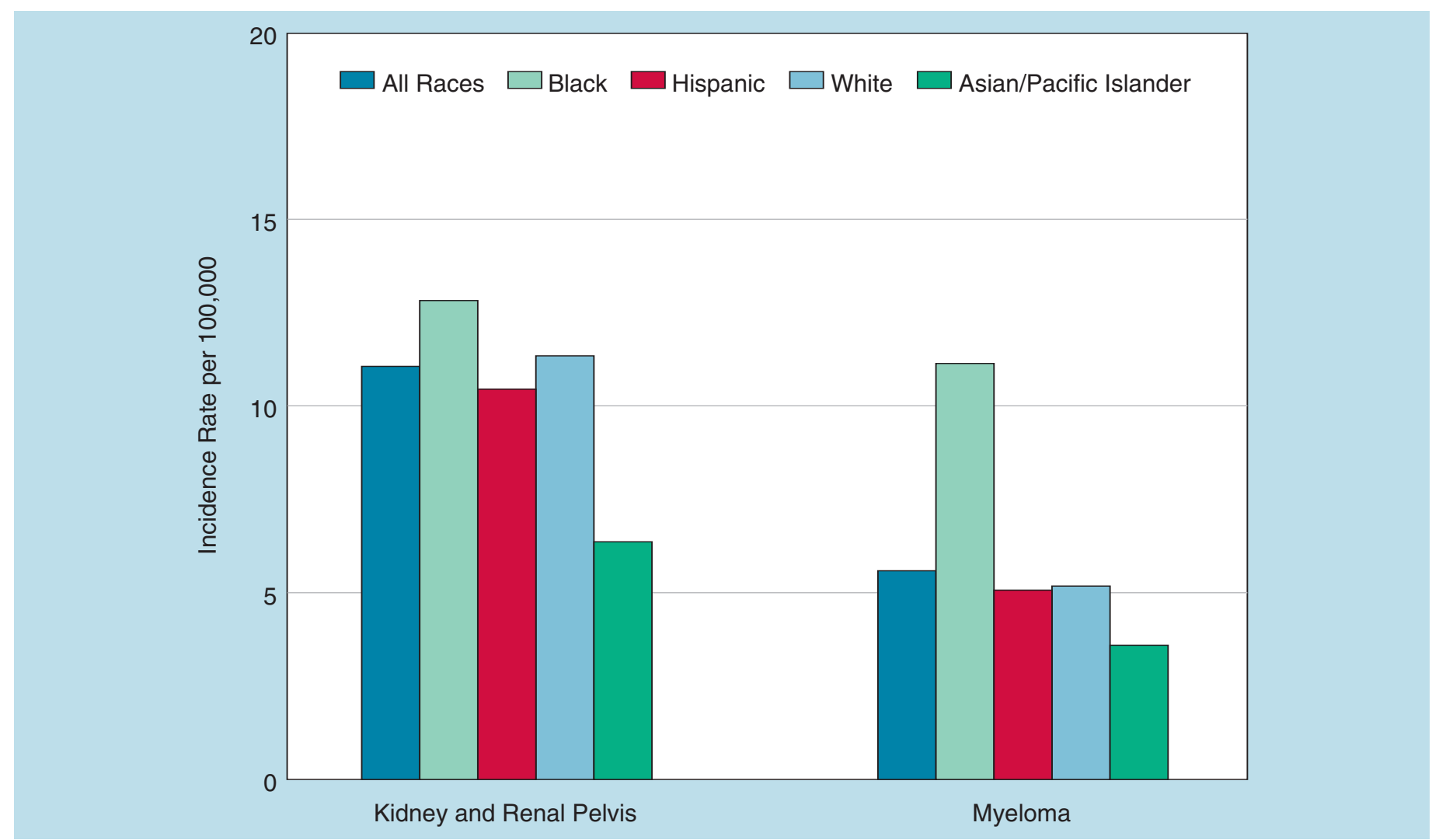

Source: SEER Cancer Statistics Review, 1975-2000.

no inherent way to rank individuals by their race, ethnicity, disability status, or sexual orientation. Certain measures of disparity cannot be used to measure or monitor disparities between groups that have no implicit ranking. For example, the slope index of inequality and the concentration and achievement indices cannot be used except in the case of education and income, because there is no inherent way to rank some social groups such as racial/ethnic groups or genders (except by their health level). In the Healthy People 2010 parlance, groups with a "natural" ordering include education and income but do not include gender, race/ethnicity, sexual orientation, disability status, and geography.

\section{The Number of Social Groups}

Should the measure of disparity include information from all social groups (i.e., the entire population), or is it sufficient to reflect only the experiences of the best- and worst-off (extreme) groups? Many empirical studies of health disparities measure disparity by comparing the extreme groups (e.g., the lowest income group compared with the highest income group). This, however, ignores the health status of other groups and additionally may only reflect the disparity between two very small population groups. For example, in 2000 there was a three-fold relative difference in death rates from melanoma of the 
skin across U.S. states. The states with the lowest (North Dakota, 1.3 per 100,000) and the highest (Wyoming, 3.7 per 100,000) rates, however, collectively accounted for only $0.4 \%$ of the U.S. population in that year. Eliminating this disparity would have little impact on reducing the population burden of melanoma mortality because only a fraction of melanoma cases reside in these two states. Additionally, although there are good reasons for focusing attention on specific comparisons, such as the disparity between blacks and whites in the receipt of treatment for cancers of similar stage (57), such pairwise comparisons do not quantify the disparity across all racial/ethnic groups, which is precisely the goal of initiatives to eliminate health disparities by the year 2010. For example, the gap between white and black men in the recent use of fecal occult blood test (FOBT) screening for colorectal cancer narrowed between 1987 and 1998 (58); however, this pairwise comparison conceals the fact that the gap between Hispanics and whites and between Hispanics and blacks increased (see Figure 8). Despite the utility of measuring disparities between two groups, pairwise comparisons may conceal important heterogeneity and thus provide a limited view in monitoring progress toward eliminating health disparities across the entire range of social groups.

\section{Figure 8. Proportion of Men Reporting Use of Screening Fecal Occult Blood Tests (FOBT), by Race and Ethnicity, 1987-1998}

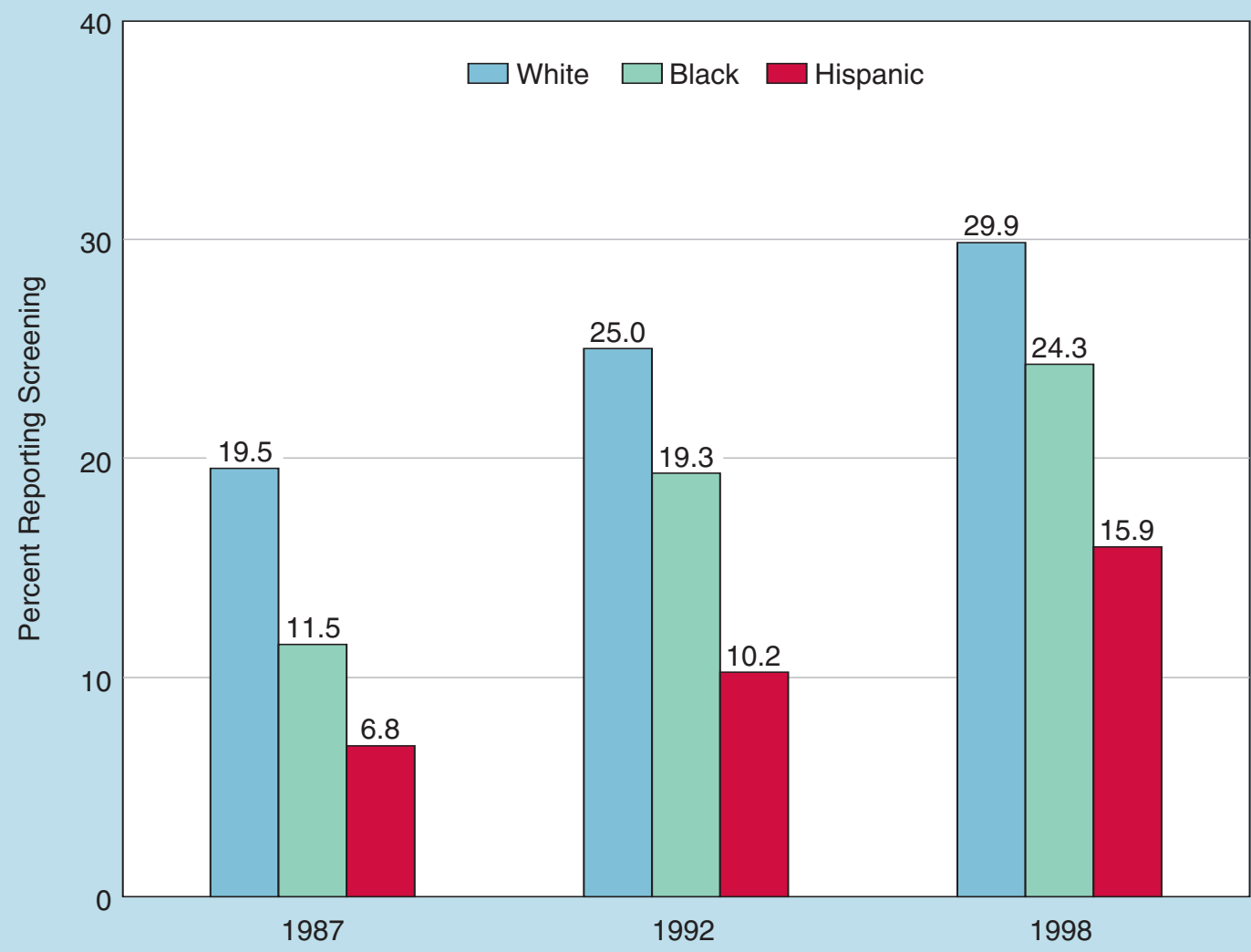

Source: Breen et al. J Natl Cancer Inst 2001;93:1704-13. 


\section{Population Size}

Should the disparity measure incorporate the size of the groups being compared? If we use a pairwise comparison of extreme groups, would it matter that one or both of those groups comprises a very small proportion of the population? For example, Pearcy and Keppel's Index of Disparity (30) gives equal weight to each group, even though the groups may represent different proportions of the population. This has important implications for monitoring disparities and is another case in which a statistical choice reflects an ethical choice. That is, the decision of whether or not to weight social groups by their population size also is a decision regarding how much weight to give individuals within each social group. For example, if we measure the disparity in prostate cancer mortality among U.S. states in 2000 without weighting states by their population size, California and Wyoming receive equal weight despite the fact that California has nearly 70 times as many males as Wyoming. Thus, in an unweighted analysis of U.S. states, individual males in California receive approximately $1 / 70^{\text {th }}$ the weight of males in Wyoming.

Another important issue in using unweighted measures of health disparity is their inability to incorporate the demographic changes that inevitably occur over time. For example, Figure 9 (page 28) shows the percentage increase in population subgroups between the 1980 and 2000 Census (59). These demographic shifts can have enormous impact on the population's health and should be factored into the assessment of health disparity. In their analysis of the effects of education on all-cause and cause-specific mortality in the American Cancer Society's Cancer
Prevention Study cohorts (CPS-I and CPS-II), Steenland and colleagues (who used ordinary least squares regression) noted that changes in the distribution of education made it difficult to compare the extent of disparity between the two populations studied (36). The proportion of the population with less than a high-school education was $20 \%$ in CPS-I and 6\% in CPS-II, while those with a college degree were $16 \%$ and $30 \%$ of the population in the two respective cohorts. In epidemiological language, the proportion of the population "exposed" changed dramatically with large population shifts out of the most disadvantaged groups. For a measure of health disparity to allow for an unambiguous comparison across time, it should be sensitive to changes in the distribution of social groups over time. This sensitivity to changes in the proportion of people exposed to disadvantageous social positions especially is important when considering the so-called "upstream" determinants of health disparities. It is commonplace in health disparity research to discuss how distal social policy affects health and health disparity. The policies and programs that define the nature of stratification in a society create educational opportunity, allocate income, and affect the types of jobs that are available. When these "upstream" social policy factors affect the nature of social stratification by reducing the number of minimally educated individuals, for instance, thus reducing the number of individuals exposed to that form of social disadvantage, then measures of health disparity should account for that change. The same situation exists when the proportion of a particular population subgroup changes over time, as in the case of the migration of Hispanics and Asian/Pacific Islanders as shown in Figure 9. 
Figure 9. Percent Change in Population Size by Race and Hispanic Origin, 1980-2000

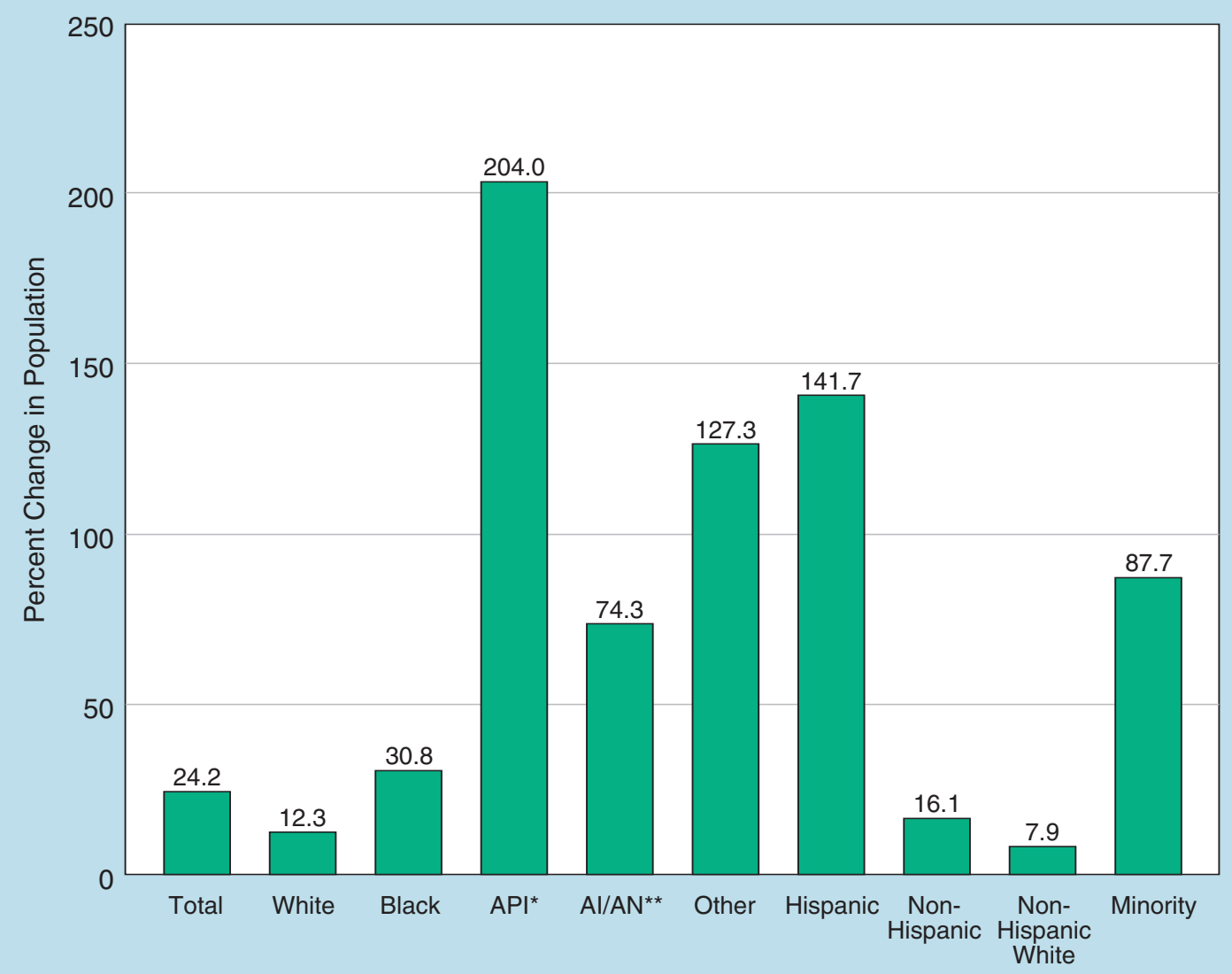

*Asian/Pacific Islander

**American Indian/Alaska Native

Source: Hobbs F, Stoops N. Demographic Trends of the 20th Century, 2002.

\section{Socioeconomic Dimension}

Another potential criterion for a measure of health disparity, first articulated by Wagstaff and colleagues (60), is whether the measure is able to capture health gradients associated with socioeconomic position. By health gradients, we mean a situation where a measure of health status either increases or decreases with increasing socioeconomic position. A good example is the increasing rate of cancer incidence among individuals living in U.S. counties with successively higher poverty rates (32). That is, is the measure sensitive to the direction of the association between social group and health? For instance, if at one time health status increases with social-group ordering and at another time health decreases with the same social-group ordering, the disparity measure will reflect this change if it is sensitive to the direction of the gradient. Of necessity, this criterion is applicable only for measuring inequality between social groups that have an inherent ranking. The lack of inherent ordering among racial/ethnic groups, for example, means that the "socioeconomic dimension" criterion cannot be applied to disparity measures used to monitor racial/ethnic health disparities. 


\section{Monitoring Over Time}

Inherent in the goals of Healthy People 2010 is the idea that we monitor progress toward the elimination of health disparities. That means it is desirable that measures of disparity are interpretable over time. This represents important challenges for the use of simple pairwise relative disparity indicators and indicators that are not population-weighted. Because both the health status within different social groups and the population distribution of social groups change over time, which together reflects the overall public health burden of health disparities, measures that are sensitive to both dimensions of change may be more suitable for monitoring disparities over time.

\section{Transfers}

The issue of how measures of disparity respond to hypothetical transfers between individuals has been an important part of evaluating the performance of income disparity measures in economics. The major test in economics is the principle of transfers-sometimes called the Pigou-Dalton condition $(61,62)$-which maintains that a transfer of income from a richer to a poorer person should result in a decrease in the measure of disparity (assuming that everyone else's income remains unchanged and the transfer is not large enough to reverse anyone's relative positions). This is an intuitively powerful and desirable notion that corresponds well with what we believe disparity measures should be able to capture. Yet, theoretically, this is a somewhat difficult concept to employ for judgments about health disparity. Is "health" a fungible good like income that can be redistributed in different ways? It is hard to imagine social mechanisms (perhaps apart from organ donation) through which a "healthy" person can directly transfer some of her health to someone who is less healthy, though it is possible to conceive of redistributing health resources. The task, however, is to measure disparities in health, not health resources.

We have noted that measuring disparity in health versus income differs in at least one important respect, namely that goods such as income or wealth are, in fact, transferable from one individual to another. One potential way to avoid this difficulty is to think of comparing disparity in two different populations (e.g., in two repeated observations of a cohort). One might then think of a transfer-like principle according to which we evaluate a measure of health disparity. If the health of every individual remains the same, but a single "healthier" person becomes less healthy and a previously "less healthy" person's health improves, the measure of health disparity should decrease (25). This seems a plausibleenough principle to warrant evaluating a measure of health disparity, but health disparities and income distributions are dissimilar in another way. Even if we are willing to put aside the issue of the literal inability to "transfer" health, it is not at all clear in the previous example that we would be willing to accept the decreased health of one person for the sake of increasing the health of another. For income, this is not a problem because it is the distribution of the good itself that is under question. Most people generally believe that it is unfair that some have enormous incomes while others live in extreme poverty. Do 
we truly believe, however, that some individuals possess more than their fair share of health? As was emphasized earlier, one of the major reasons for the increasing focus on health inequalities is not simply that some are healthy while others are sick. It is that some kinds of individuals or the members of some social groups are healthy while other kinds are sick. It is the normative distinction between the kinds of healthy or unhealthy individuals that drives our concern that health differs so markedly by social group. The concern over health disparities then, at least in the current historical period, is not that there are health differences in society but that these health differences systematically covary with membership in particular social groups.

\section{Subgroup Consistency}

Generally, this criterion says that if the measure of overall disparity includes, for example, three groups, and disparity within two groups remains unchanged while increasing within the third, the measure of disparity should increase. This is of most relevance when we are interested in measuring the overall disparity (i.e., across the entire population). For instance, suppose we were examining alcohol consumption at two points in time in a population composed of two social groups (rich and poor). At each time, both the size of these groups and their average alcohol consumption remain constant, but the disparity in consumption increases within the poor and remains constant within the rich. Subgroup consistency requires that any measure of overall disparity also should register an increase in this scenario. This is not likely to be an important criterion for health disparity measures in the context of Healthy People 2010 because it does not focus on health disparities within subgroups of a social group (e.g., within the poor).

\section{Decomposability}

Decomposition as a property of statistical measures is common in both economics and epidemiology. In economics, it typically refers to the ability to decompose a measure of disparity by sources of income or into between-group and within-group partitions (40). Decomposable disparity measures are seen as advantageous as they can offer information about the sources of increasing or decreasing disparity as indicated in a summary statistic. In public health, decomposition often is used to capture differences in summary rates. For example, a difference in age-adjusted mortality rates between two populations can be "decomposed" into differences between mortality rates and differences in age structure.

\section{Scale Independence}

Scale independence (or invariance) often is seen as a desirable property of disparity measures. It often is argued that, all else being equal, if everyone's health "doubles," the disparity measure should remain unchanged. It is arguable, however, whether for public health, where we also are concerned about the absolute level of ill health, this is a desirable property. 


\section{Transparency/Interpretability for Policy Makers}

Finally, it seems salient that an interpretability criterion be included as a factor in decisions about measures of disparity. For instance, despite other desirable properties, the actual value of more sophisticated summary measures such as the Concentration Index have no obvious interpretation and thus may make communicating health disparity indices to the community and policy makers potentially more difficult. Thus, the extent to which different measures of disparity can be captured graphically to aid communication might be important in deciding which measures are most appropriate for monitoring cancer-related health disparities. Perhaps the use of real-time graphical displays of changes in outcomes of interest may aid the understanding of health disparity. This dimension of health disparity monitoring should not be underestimated, as evidenced by the lack of general application of more sophisticated disparity measures in health disparity research. 


\section{Measures of Health Disparity}

This section reviews most of the statistics that are available to measure health disparities. The goal is to provide a brief overview of each measure, followed by the method of calculation and statistical interpretation and, often, an example of its actual or potential use for measuring disparities in cancer-related health objectives.

Note that there are methods to calculate indicators of precision (e.g., 95\% confidence interval) for all of the measures reviewed here. These can be found in the source publications detailed in the references. Although issues of variability and precision are important, they are not germane to the choice of disparity measure because they ultimately derive from the precision of the underlying rates, prevalence, and proportions that are used to generate a particular disparity measure.

\section{Measures of Total Disparity}

A measure of "total disparity" in health is a summary index of health differences across a population of individuals. Generally, measures of total disparity do not account for social grouping and have been used chiefly by health economists (see, for example, 25,49). They are an important first step in understanding the scope of health variation in a population and have advantageous properties for monitoring trends, particularly for cross-country comparisons. They do not, however, inform about systematic variation in health among population subgroups, which is inherent in the Healthy People 2010 health disparity initiatives. The measurement of health disparity as total disparity is associated most closely with and endorsed by the WHO as a component of its general framework for routinely assessing the performance of health systems in different countries. The WHO, however, is not the only advocate of measuring total disparity. Some health economists also advocate for the measurement of total health disparity $(25,63,64)$ as the primary form of assessing health inequalities.

A number of criticisms have been levied at this kind of measure, primarily because it does not distinguish among individuals from different social groups $(51,53,54,65)$. In addition, empirical investigations using measures of total disparity appear difficult to interpret $(54,66,67)$. Those who endorse this measure often cite as their primary justification the weighty normative choices that must be made to measure health differences between social groups and note that the absence of such a priori choices makes disparity between individuals a more "objective" measure of health disparity. We recognize that Healthy People 2010 specifically calls for social-group monitoring and not total variation, but we include measures of total group disparity because they are prominent in the overall framework of efforts to monitor global health disparity and because they provide an essential context for understanding the 
"decomposition" of health disparity measures, as described below.

\section{Individual-Mean Differences}

Individual-mean difference (IMD) measures of health disparity calculate the difference between the health of every individual in the population and the population average. The general formula for the class of individual/mean difference measures is given by Gakidou and colleagues (49) as:

$$
\operatorname{IMD}\left(\boldsymbol{\alpha}, \boldsymbol{\beta}=\frac{\sum_{i=1}^{n}\left|y_{i}-\mu\right|^{c}}{{ }_{n \mu} \beta}\right.
$$

where an individual i's health is $y_{i^{\prime}} \mu$ is the mean health of the population, and $n$ is the number of individuals in the population. The parameters $\alpha$ and $\beta$ specify, respectively, the significance attached to health differences at the ends of the distribution relative to the mean and whether the individual-mean difference is absolute or relative to the mean health of the population. For instance, large values of $\alpha$ emphasize greater deviations from the mean, and larger values of $\beta$ emphasize relative disparity because of heavier weighting of the mean. Those familiar with basic statistics will note that, when $\alpha=2$ and $\beta=0$, the IMD simply is the variance; and when $\alpha=2$ and $\beta=1$, the $I M D$ is the coefficient of variation (49). Similar to many other disparity measures, the $I M D$ is a "dimensionless" index that is not measured in units because it always is relative to the mean in the population.

\section{Inter-Individual Differences (IID)}

The IID measures health differences between all individuals in the population and is consistent with the Gini coefficient but may be weighted in accordance with differential aversion to disparity (i.e., the value chosen for $\alpha$ ). These measures are different from the $I M D$ class because they compare every individual in the population with every other individual in the population, whereas the $I M D$ measures disparity relative to the population average. It should be clear that different measures of disparity implicitly express different perspectives on which aspects of disparity should be emphasized in the measure. The class of inter-individual difference measures is (49):

$\operatorname{IID}\left(\boldsymbol{\alpha}, \boldsymbol{\beta} \frac{\sum_{i=1}^{n} \sum_{j=1}^{n}\left|y_{i}-y_{j}\right|^{c}}{2 n^{2} \mu^{\beta}}\right.$

where $y_{i}$ is individual $i$ 's health, $y_{j}$ is individual $j$ 's health, $\mu$ is the mean health of the population, and $n$ is the number of individuals in the population. The parameters $\alpha$ and $\beta$ are defined as for the $I M D$ above, and it is worth noting that, when $\alpha=2$ and $\beta=1$, the IID is equal to the more well-known Gini coefficient. Gakidou and King have used this disparity measure (with $\alpha=3$ and $\beta=1$ ) to compare total disparity in child survival among 50 countries (68). Weighting $\alpha=3$ implies that the measure should be more sensitive to larger than smaller pairwise deviations between individuals and thus reflects additional concern about larger health differences between individuals. To our knowledge, there is only one study of total disparity that uses data from the United States (69). 


\section{Measures of Social-Group Disparity}

The measures of total variation described above have a number of merits, including their ability to make unambiguous health disparity comparisons between populations and over time. In defining health disparity as disparity between individuals instead of between social groups, such measures avoid the difficulty of comparability of groups between populations or over time (50). This makes them particularly attractive for crosscountry comparisons, in which defining comparable social groups is challenging because of differences in how social groups are classified in different countries (70).

The disparity goals of Healthy People 2010, however, explicitly are goals that relate to socialgroup differences in health. It is an open question as to whether measures of total disparity and social-group disparity are "better" or "worse" disparity measures, but the concern among health policy makers in the United States specifically is expressed in terms of social-group differences in health. Measures of total disparity therefore are insufficient for monitoring progress toward eliminating cancer-related health differences among social groups in the United States.

\section{Pairwise Comparisons}

Simple comparisons of some health indicator between two groups in a population (so-called pairwise comparisons) clearly are one of the most straightforward ways to measure progress toward eliminating disparities between groups. For example, age-adjusted incidence rates of lung cancer for black and white females in 1973 were, respectively, 23.6 and 20.4 per 100,000. By 1999, rates for both groups had increased, to 57.0 for blacks and 52.3 for whites (71). It would seem easy enough to answer the question: Did blackwhite disparity grow from 1973 to 1999 ? Unfortunately, however, the answer depends on the measure of disparity. If the disparity measure is the absolute difference between the black and white rates, then we would conclude that the black-white disparity increased from 3.2 to 4.7 . If the disparity measure is the relative difference between the black and white rates (i.e., black rate $\div$ white rate), however, we would conclude the opposite because the relative disparity decreased from 1.16 to 1.09. Both answers are correct. This has been a source of continuing confusion and sometimes unresolved debate in the health disparities literature $(72,73)$ and, although most of the empirical work in health disparities has been in terms of "relative disparity," it should always be kept in mind that large relative differences can mask very small differences in absolute terms, which can be misleading with respect to the disparity's population-health impact. Conversely, there may be situations where large relative disparities may be viewed as grossly unjust, despite the fact that they reflect small absolute differences.

\section{Absolute Disparity}

The absolute disparity between two health-status indicators is the simple arithmetic difference. It is calculated as:

$A D=r_{1}-r_{2}$

where $r_{1}$ and $r_{2}$ are indicators of health status in two social groups. In this case, $r_{2}$ serves as the reference population, and the $A D$ is expressed in 
the same units as $r_{1}$ and $r_{2}$. A typical disparity measure that uses the absolute difference between two rates for an entire population is the range, where case $r_{1}$ above corresponds to the leasthealthy group and $r_{2}$ to the most-healthy group.

\section{Relative Disparity}

For the same pairwise group comparison in equation [3], we also can divide $r_{1}$ into $r_{2}$ to calculate the relative disparity as:

$$
R D=r_{1} / r_{2}
$$

where, again, $r_{2}$ is the reference population. This rate ratio can be transformed easily into a percentage difference by multiplying the ratio by 100 . Figure 10 shows the absolute and relative black-white disparity for prostate and stomach cancer incidence from 1992-1999. Clearly, there is a much larger absolute disparity in prostate cancer incidence because the rates for both groups are relatively high compared to the stomach cancer rates; however, the relative disparity is larger for stomach cancer.

\section{Regression-Based Measures}

One drawback of the pairwise comparison measures of disparity is that, when a social group has more than two subgroups (as most do), information on the other groups is ignored. Normally it is desirable to use as much of the information present in the data as possible. If we

Figure 10. Absolute and Relative Black-White Disparities in Prostate and Stomach Cancer Incidence, 1992-1999

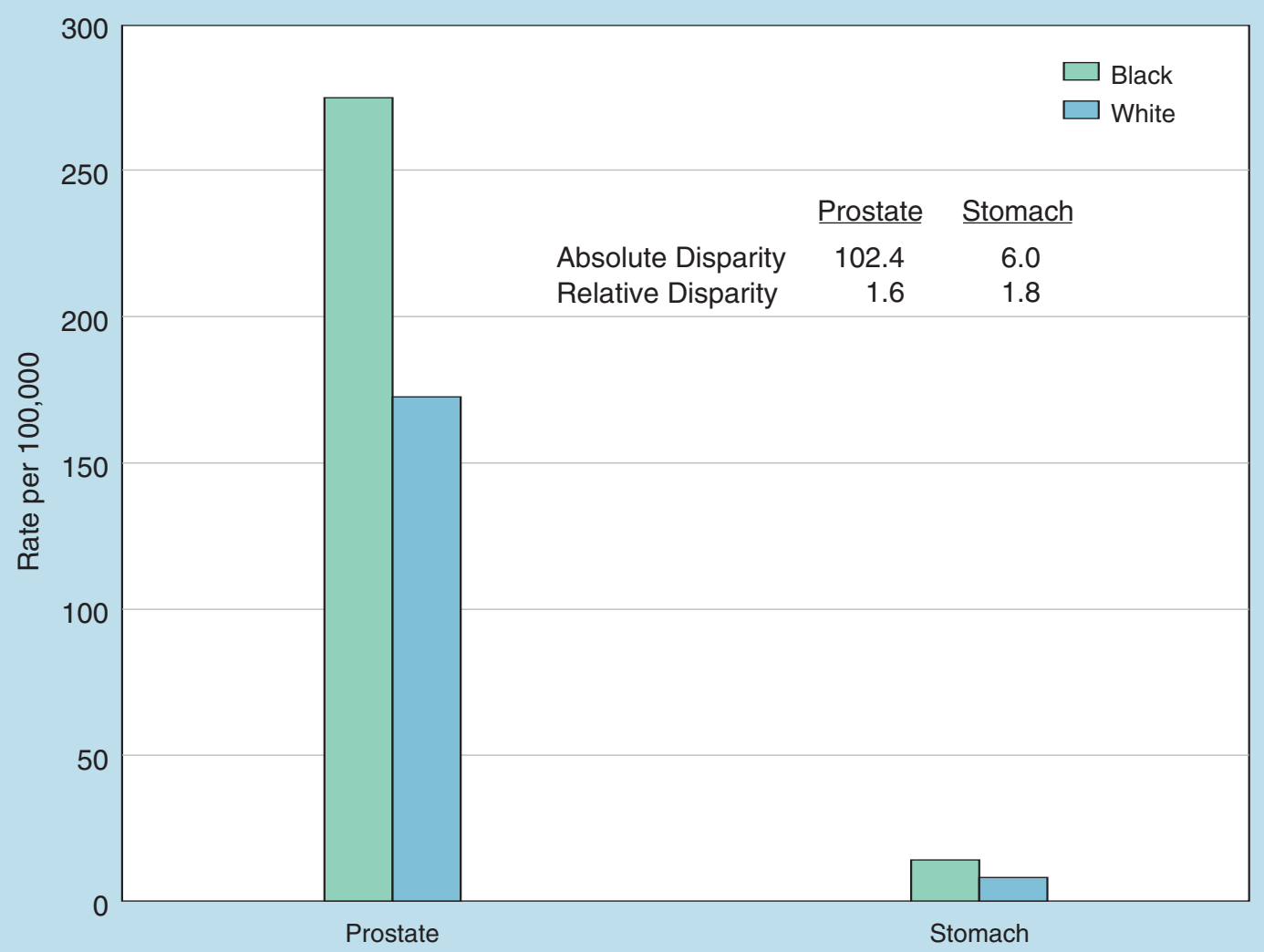


compare the "best" group to the "worst" group, we effectively ignore the information on the health status of all the groups in between, aside from knowing that they fall somewhere between the best and worst groups. One possible solution would be to calculate a series of $(j-1)$ pairwise comparisons for $j$ groups using one group as the reference point, or $j$ pairwise comparisons using an external reference point. Although feasible, as the number of groups, time periods, or both increases, attempting to evaluate the disparity trend may become complicated in terms of summarizing the many pairwise comparisons. To overcome this limitation and make use of the information for all groups, one might consider calculating a summary measure of disparity. This choice, however, undoubtedly involves additional complexity and assumptions that must be traded off against the insights about disparity gleaned from the use of a summary measure (74).

\section{Simple Linear Regression}

If one is willing to assume that the relationship between social group and health status is linear (i.e., that each step up the social-group scale results in an equivalent health gain/loss), then a potential way to include information on all of the groups is to calculate a summary measure of disparity using regression. One way of writing this is:

$y_{i}=P+P X_{i}$

where $y_{i}$ is a measure of health status for individual $i, \beta_{0}$ is the value of the health variable when $X_{i}$ is 0 (e.g., if $X_{i}$ is a continuous measure of income, then $\beta_{0}$ is the health status of an individual with zero income), $X_{i}$ indexes social group, and $\beta_{1}$ is the summary measure of disparity. In general terms, $\beta_{1}$ is equal to the covariation of $X_{i}$ and $y_{i}$ expressed in terms of the variance of $X_{i}$. The specific interpretation of $\beta_{1}$ depends on the particular health status measure used and the specification of the model. If $y_{i}$ is an untransformed health status measure-for example, BMI-then $\beta_{1}$ is the absolute increase in BMI associated with a one-unit change in social group and is referred to as a Regression-Based Absolute Effect or RAE (70). It is an absolute measure because it is expressed in the same units as the quantity of health measured in $y_{i}$. Continuous types of health outcomes, however, are relatively less common in the area of cancerrelated data. More likely are noncontinuous types of health data (e.g., the presence or absence of cancer, receipt or nonreceipt of screening), where the linear relationship in equation [5] applies to some transformation of the dependent variable $y_{i}$. For transformations of the dependent variable $y_{i}$ (e.g., the logarithmic or logit transformation), $\beta_{1}$ then becomes a relative-risk (logarithmic transformation) or odds-ratio (logit transformation) and is interpreted as the proportional increase in health status for a one-unit change in social group and referred to as a Regression-Based Relative Effect or RRE (70). Figure 11 (page 38) graphically shows a simple regression-based disparity measure, applied in this case by Steenland et al. to the risk of lung cancer among men of different education groups (grammar, some high school, high-school graduate, some college, college graduate) in the 1982-1996 Cancer Prevention Study II (36). The $y$-axis is the risk of mortality relative to those completing graduate school (whose relative risk is by definition equal to 1.0), the $x$-axis is the approximate number of years of education for each education group ( $X_{i}$ in equation [5]), and the fitted line indicates the linear decrease in relative 
risk-which Steenland and colleagues reported as about 10\%-for each 1-year increase in the number of years of education. (It is important to note that, for ease of presentation, the plotted points in Figure 11 show only the average relative risk for each education group. The actual regression equation is performed on all 500,000 or so individuals in the CPS-II.) Relative-effect measures also may be transformed into absolute effect measures by applying them to the rates of health in the referent social group. An additional drawback to the $R A E$ and $R R E$ is that the assumption of linearity between health and social group may be problematic. For example, while Kunst and colleagues find linear associations between education and self-rated health (75), Manor et al. report nonlinearity between education and a number of chronic conditions (76), and Backlund and colleagues report a nonlinear association between income and mortality (77).

Slope Index of Inequality

The regression-based methods outlined above, subject to the assumptions of the model, work well for calculating a summary measure of health disparity at a single point in time. As noted above, however, over time the distribution of the population in various social groups may change

Figure 11. Example of a Simple Regression-Based Disparity Measure

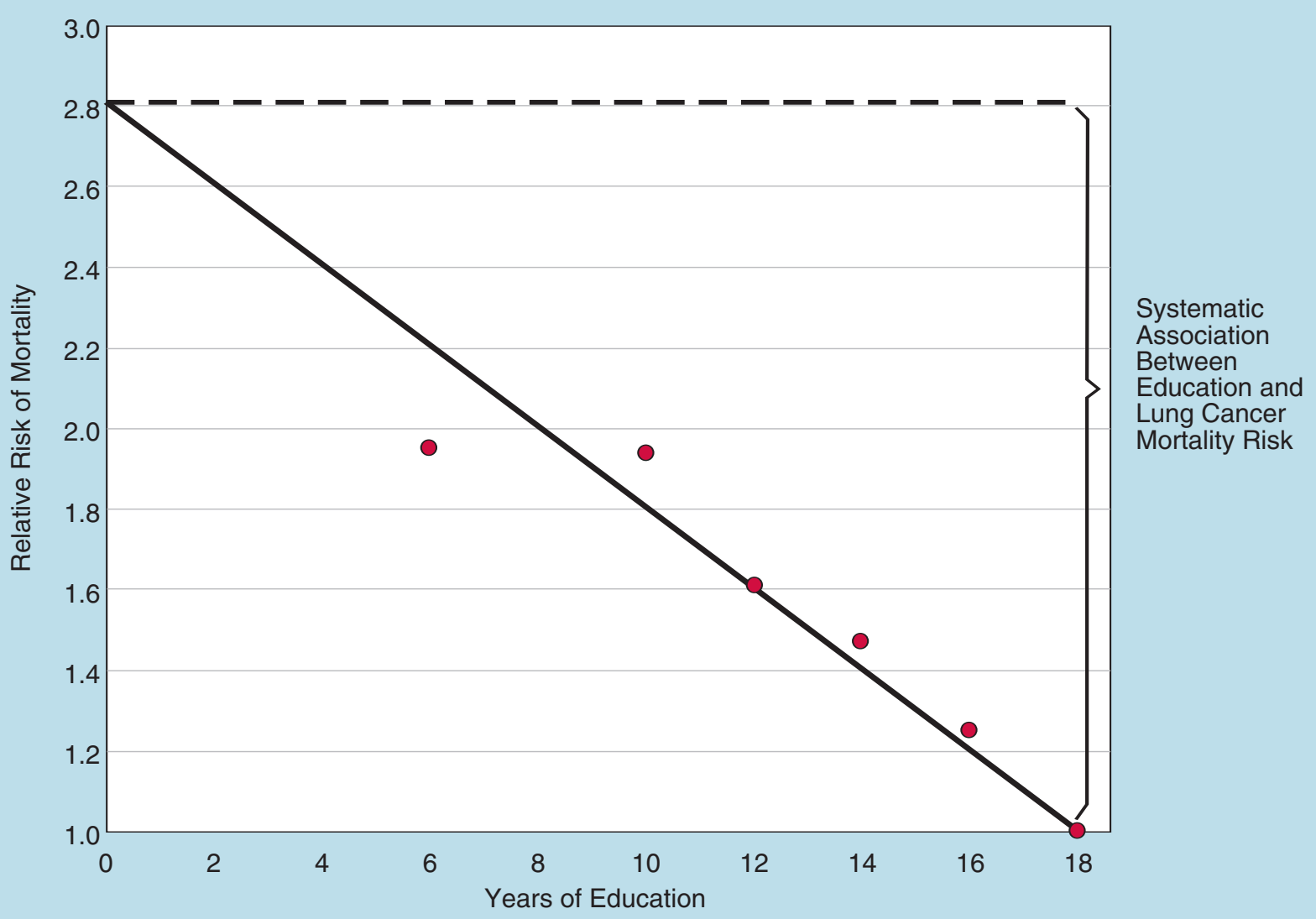

Source: Adapted from Steenland et al. Am J Epidemiol 2002;156:11-21. 
drastically, and it would be advantageous for a measure of health disparity to be sensitive to such changes. One measure that does so is the Slope Index of Inequality (SII). To calculate the SII, the social groups first are ordered from lowest to highest. The population of each social-group category covers a range in the cumulative distribution of the population and is given a score based on the midpoint of its range in the cumulative distribution in the population. For example, in the 2001 NHIS those with an incometo-poverty ratio of less than 0.5 (approximately $<\$ 9,000$ for a family of four) were $3.45 \%$ of the population, and those in the next highest income group-with an income-to-poverty ratio of 0.5 to 0.74 -comprised $3.02 \%$, in which case the lowest group is assigned a score of $[0+(.0345-0) / 2]=$ .0173 , and the next lowest group is assigned a score of $[.0345+(.0647-.0345) / 2]=.0496$.

Health status then is plotted against this midpoint socioeconomic category variable, and a regression line is fitted to the data. The SII thus is similar to the regression-based methods above, but differs because it uses the midpoint of the cumulative social group distribution and because it (usually) is based on grouped data and is a weighted index, where the weights are based on the size of the social groups. By weighting social groups by their population share, the SII is able to incorporate changes in the distribution of social groups over time that affect the population health burden of health disparities. Figure 12 (page 40) shows the predicted slope for the income disparity (based on income-to-poverty ratio) in current smoking for the United States in 2001. Note that, in Figure 11, the location of the data points on the $X$-axis is based on the estimated number of years of education, whereas in Figure 12, the location is based on the group's share of the population. This reflects the fact that the education groups actually comprise different proportions of the population distribution. Formally, the SII, which was introduced by Preston, Haines, and Pamuk (78), may be obtained via regression of the mean health variable on the mean relative rank variable:

$\bar{y}_{j}=P+P \bar{R}_{j}$

where $j$ indexes social group, $\bar{y}_{j}$ is the average health status, $\bar{R}_{j}$ is the average relative ranking of social group $j, \beta_{0}$ is the estimated health status of a hypothetical person at the bottom of the social group hierarchy (i.e., a person whose relative rank $R_{j}$ in the social group distribution is zero), and $\beta_{1}$ is the difference in average health status between the hypothetical person at the bottom of the social group distribution and the hypothetical person at the top (i.e., $R_{j}=0$ vs. $R_{j}=1$ ). Because the relative rank variable is based on the cumulative proportions of the population (from 0 to 1 ), a "one-unit" change in relative rank is equivalent to moving from the bottom to the top of the social group distribution. Because this regression is run on grouped data (as opposed to individual data as in equation [5]), it is estimated via weighted least squares, with the weights equal to the population size $n_{j}$ of group $j(60)$. The coefficient $\beta_{1}$ in equation [6] is the SII, which is interpreted as the absolute difference in health status between the bottom and top of the socialgroup distribution. Thus, the regression equation in Figure 12 shows that the absolute difference in the prevalence of smoking across the entire 
distribution of income is -18.1 percentage points. The same regression also may be run on individual data (as in equation [5]), but replacing $X_{i}$ with $R_{i}$, with $R_{i}$ being an individual's relative rank in the social-group distribution. In this case, the data would be self-weighting and could be estimated by ordinary least squares.

Relative Index of Inequality

The SII discussed above is a measure of absolute disparity. Dividing this estimated slope by the mean population health, however, provides a relative disparity measure, the Relative Index of Inequality or RII (79):

$R I I=S I I / \mu=\not P / \mu$

where $\mu$ is mean population health and the SII is the estimate of $\beta_{1}$ from equation [6]. Its interpretation is similar to the SII, but it now measures the proportionate (in regard to the average population level) rather than the absolute increase or decrease in health between the highest and lowest socioeconomic groups. In the income and smoking example seen in Figure 12, the RII is calculated as $-18.1 / 24.6=-0.74$, indicating that a

Figure 12. Income-Based Slope Index of Inequality for Current Smoking, NHIS, 2002

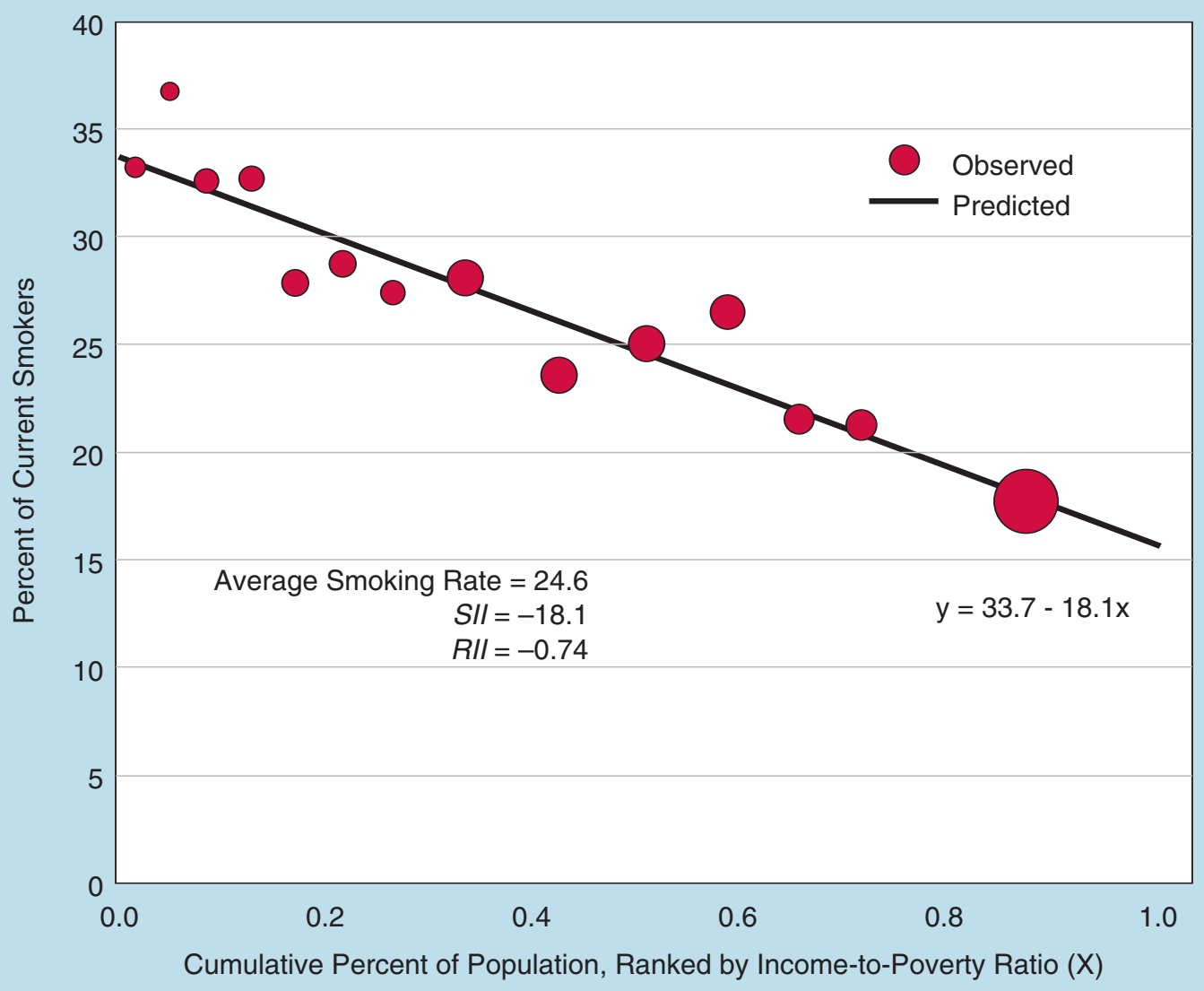


move from the bottom to the top of the income distribution is associated with a $74 \%$ decline in the prevalence of smoking. Kunst and Mackenbach (70) modified this definition of the RII slightly by dividing the estimated health of the hypothetical person at the bottom of the socialgroup distribution by the estimated health of the hypothetical person at the top of the social-group distribution:

$R I I_{K M}=\frac{\not P}{\beta}+\beta=\frac{\not P}{\beta+S I I}$

where $\beta_{0}$ and $\beta_{1}$ are defined as in equation [6]. From Figure 12, this is calculated as 33.7/(33.7 $18.1)=2.16$, indicating that the rate of smoking is 2.16 times higher at the bottom of the income distribution than at the top. Thus, the KunstMackenbach RII is more like a traditional relative risk measure in that it compares the health of the extremes of the social distribution, but it is estimated using the data on all social groups and is weighted to account for social-group sizes. As noted above, the use of the SII and RII indices (as well as the Health Concentration Index discussed below) depends on having a social-group classification scheme that is hierarchical. This seems straightforward with respect to education and income, but social-group classifications based on occupation may be somewhat more challenging because there inherently is more ambiguity in the ranking of occupations (80). In their international study of occupational mortality differences, Kunst and Mackenbach (81) note this difficulty as a possible explanation for the lack of consistency of their results with those of Wagstaff for the size of disparity in Finland versus England and Wales (60).

\section{Population Impact Measures}

\section{Population Attributable Risk}

The Population Attributable Risk (PAR) and its relative analogue, the $P A R \%$, are longstanding epidemiologic measures of the population burden that is associated with differential health between groups. Although typically applied to groups defined by their exposure status (e.g., comparing smokers with nonsmokers), it also may be applied in the context of health differences between social groups (poor vs. nonpoor). It is a summary of differences between each social group's health and the health of the "best" group. For example, it indicates the absolute (or relative, in the case of the $P A R \%$ ) aggregate health improvement that would be obtained if all education groups had the health of the healthiest education group. The basic formulas for $P A R$ and $P A R \%$ as health disparity indicators (70) are:

$P A R=r_{p o p}-r_{r e f}$
$P A R \%=\frac{r_{\text {rop }}-r_{\text {ref }}}{r_{p o p}}$

where $r_{p o p}$ is the rate in the total population and $r_{\text {ref }}$ is the rate of health or disease in the reference group, typically the best-off social group. While not immediately clear from the above formula, the $P A R \%$ in fact is a population-weighted (by social-group size) sum of the relative risks ( $R R \mathrm{~s})$ for each group (13) and also may be written as:

$P A R \%=\frac{\sum p_{j}\left(R R_{j}-1\right)}{\sum p_{j}\left(R R_{j}-1\right)+1}$

where $p_{j}$ is the group's population share and $R R_{j}$ is the relative rate of group $j$ compared to the reference group. To see this, note that we could 
substitute $r / r_{\text {ref }}$ for $R R_{j}$ and $r_{\text {ref }} / r_{\text {ref }}$ for 1 in equation [11] and multiply through by $r_{\text {ref }}$ to get:

$P A R \%=\frac{\sum p_{j}\left(r_{j}-r_{\text {ref }}\right)}{\sum p_{j}\left(r_{j}-r_{\text {ref }}\right)+r_{\text {ref }}}$

Because $\sum p_{j} r_{j}=r_{p o p}$ and $\sum p_{j} r_{r e f}=r_{\text {ref }}$, equation [12] reduces to equation [10]. The PAR\% varies from 0 to 100 and is interpreted as the percent improvement in the health of the total population that would be achieved if all social groups had the rates of health in the best-off social group, a commonly used metric for describing the impact of health disparities. For example, Navarro argues that "the intervention that would add the most years of life to the populations of Spain or the USA (or, for that matter, any other country) would be one that would lead to all social classes having the same mortality rates as those at the top" (65, page 1701). In the example in Figure 13, the population average rate of cervical cancer would be improved by $28 \%$ if all social groups had the rate experienced by American Indians and Alaska Natives.

\section{Figure 13. Example of the Population-Attributable Risk Percent}

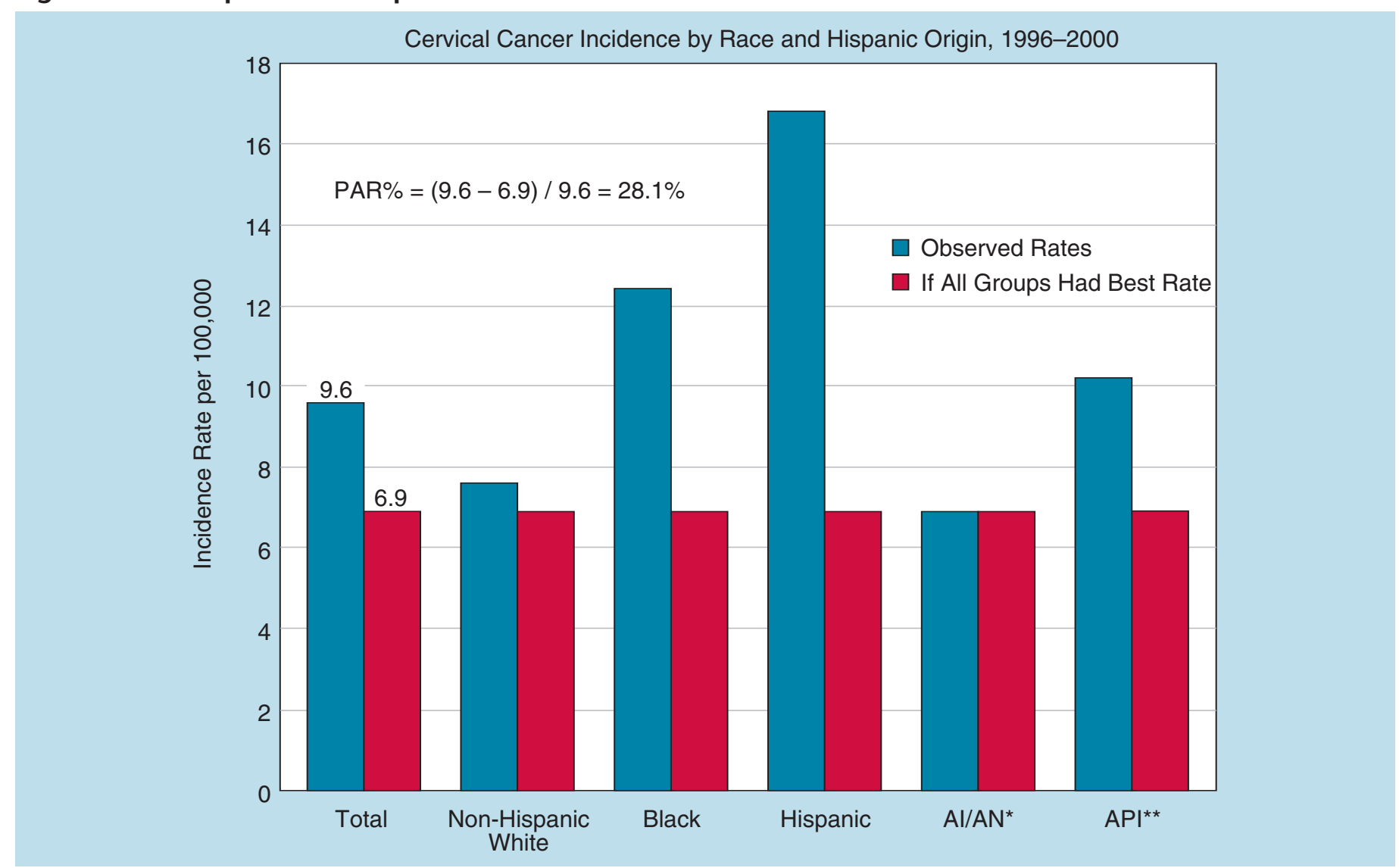

*American Indian/Alaska Native

**Asian/Pacific Islander

Source: SEER Cancer Statistics Review, 1975-2000. 


\section{Index of Dissimilarity}

The Index of Dissimilarity (ID) originally was developed as a measure of residential segregation of population groups (82). For example, in the context of black-white segregation among neighborhoods within a city, the ID measures the proportion (using the relative version) or number (using the absolute version) of blacks (or whites) that would have to move to a different neighborhood to achieve a racial distribution in each neighborhood that was similar to that of the city as a whole. As such, the ID is a summary measure of the disparity between each neighborhood's racial composition and the racial composition of the city as a whole. Similarly, in the context of health disparity measurement, we can think of the $I D$ as a summary measure of the disparity between, for example, each racial group's cancer rate and the cancer rate of the whole population. In this case, the $I D$ would be interpreted as the number or proportion of cancer cases that would have to be redistributed across racial groups for each group's cancer rate to be the same as the rate in the whole population. The formula for the relative $I D$ with respect to health is given in Wagstaff and colleagues (60) as:

Relative $I D=\frac{1}{2} \sum_{i=1}^{J}\left|s_{j h}-s_{i p}\right|$

where $j$ indexes social groups, $s_{i h}$ is the jth group's share of health (e.g., share of all cancer cases), and $s_{i p}$ is the jth group's share of the total population. According to Kunst and Mackenbach (70), equation [13] is the relative version of the $I D$. The relative $I D$ compares how each social group's share of the population's health compares with its share of the total population and represents the proportion of all cases (e.g., the proportion of all cancer cases) that would have to be redistributed across social groups so that each group has the same rate as the total population. The absolute version of the ID is calculated as:

Absolute $I D=\frac{1}{2} \sum_{j=1}^{J}\left|d_{j}-p_{j} r_{\text {pop }}\right|$

where $d_{j}$ and $p_{j}$ are, respectively, the observed number of cancer cases and the population of the th social group, $r_{p p p}$ is the cancer rate in the total population, so that $p_{j} r_{p p}$ is the expected number of cancer cases that would be observed if group $j$ had the same cancer rate as the total population. One could also derive the absolute version of the $I D$ by multiplying the relative $I D$ by the total number of cases to determine the absolute number of cases that need to be redistributed across groups.

Table 1 on page 44 shows how one might calculate the absolute and relative ID for esophageal cancer incidence among working-age (ages 25-64) racial groups during 1992-2000. A comparison of columns (3) and (5) shows that the share of cancer cases is lower than the share of the SEER population for all groups except blacks, who represent $13.5 \%$ of all esophageal cancer cases but only $10.5 \%$ of the population. Similarly for the absolute $I D$, a comparison of columns (2) and (6) shows that if all groups experienced the population rate of esophageal cancer, more cases would be observed for all groups except for blacks. The relative $I D$ in this case is 3.4 , which means that $3.4 \%$ of the 17,186 cases of esophageal cancer need to be redistributed across racial groups to eliminate the racial disparity. In absolute terms, this means redistributing 592 cases of esophageal cancer. 
Table 1. Incidence of Esophageal Cancer, Ages 25-64 by Race, 12 SEER Registries, 1992-2000

\begin{tabular}{|c|c|c|c|c|c|c|c|c|}
\hline Race & $\begin{array}{l}\text { Rate } \\
\text { (1) }\end{array}$ & $\begin{array}{l}\text { Cases } \\
\text { (2) }\end{array}$ & $\begin{array}{c}\% \text { of } \\
\text { T otal } \\
\text { Cases } \\
\text { (3) }\end{array}$ & $\begin{array}{r}\text { Population } \\
\text { (4) }\end{array}$ & $\begin{array}{l}\% \text { of } \\
\text { T otal } \\
\text { Population } \\
\text { (5) }\end{array}$ & $\begin{array}{r}\text { Cases if } \\
\text { No } \\
\text { Disparity } \\
\text { (6) }\end{array}$ & $\begin{array}{c}\text { ID } \\
\substack{\text { Relative } \\
I_{(3)}-} \\
(5) \mid\end{array}$ & 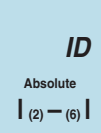 \\
\hline American Indian/Alaska Native & 5.7 & 133 & 0.8 & $2,316,609$ & 1.3 & 226 & 0.5 & 93 \\
\hline Asian/Pacific Islander & 8.7 & 1,648 & 9.6 & $18,850,492$ & 10.7 & 1,835 & 1.1 & 187 \\
\hline Black & 12.9 & 2,395 & 13.9 & $18,518,113$ & 10.5 & 1,803 & 3.5 & 592 \\
\hline White & 9.5 & 13,010 & 75.5 & $136,864,686$ & 77.5 & 13,323 & 1.8 & 313 \\
\hline$T$ otal & 9.7 & 17,186 & 100.0 & $176,549,900$ & 100.0 & 17,186 & $3.4 \%$ & 592 \\
\hline
\end{tabular}

\section{Index of Disparity}

The Index of Disparity, which we will abbreviate as $I D_{i s p}$ to distinguish it from the Index of Dissimilarity $(I D)$, summarizes the difference between several group rates and a reference rate and expresses the summed differences as a proportion of the reference rate. This measure was formally introduced by Pearcy and Keppel (30) and is calculated as:

$I D_{\text {isp }}=\left(\sum_{j=1}^{I-1}\left|r_{j}-r_{\text {ref }}\right| / J\right) / r_{\text {ref }} \times 100$

where $r_{j}$ indicates the measure of health status in the jth group, $r_{\text {ref }}$ is the health status indicator in the reference population, and $J$ is the number of groups compared. Although in principle any reference group may be chosen, the authors recommend using the best group rate as the comparison because that represents the rate desirable for all groups to achieve. In this case, it is not necessary to take the absolute value of the rate differences because they all will be positive. Other potential reference rates include the total population rate, the average of group rates, or some external target rate. A similar disparity measure was developed by Gaswirth (83), but it weights each group's deviation from the best rate by its population size, so that the disparity index (U) becomes:

$$
U=\sum_{j=1}^{I-1} p_{j}\left(r_{j}-r_{\text {ref }}\right)
$$

where $p_{j}$ is each group's population size. In this case, $U$ is calculated as the weighted sum of the health difference between each group and the reference group. Similar to the Index of Disparity, above, this value also can be expressed relative to the health status of the total population, which Gaswirth defines as $G=U \div r_{p p p}$. For example, Gaswirth applied this disparity measure to rates of mammography screening among non-Hispanic white, non-Hispanic black, Hispanic, and Other women ages 50-65 in the 2000 NHIS (83). The overall screening rate was $78.6 \%$, and Figure 14 (page 45) shows that white women had the highest rates of screening $(81 \%)$. The fraction of the entire population that is "underserved" ( $U$, the shaded area in Figure 14) in this case is $2.04 \%$, and if the population screening rate were increased by $G=2.6 \%$ and targeted to minority women, the screening disparity would have been eliminated. This measure has the additional desirable feature of intuitive graphical representation. Although not immediately clear from equation [16], however, it should be noted 
that, in practice, when the reference group is the group with the best rate, Gaswirth's measure $U$ is equivalent to the PAR described above, and $G$ is equivalent to the $P A R \%$ because their calculations are identical to the $P A R$ and $P A R \%$.

\section{The Between-Group Variance}

The variance is a commonly used statistic that summarizes all squared deviations from a population average. In the case of grouped data, this is the Between-Group Variance $(B G V)$, and it is calculated according to the following formula that squares the differences in group rates from the population average and weights by their population sizes:

$B G V=\sum_{j=1}^{J} p_{j}\left(y_{j}-\mu\right)^{2}$

where $p_{j}$ is group $j$ 's population size, $y_{j}$ is group $j$ 's average health status, and $\mu$ is the average health status of the population. The Between-Group Variance may be a useful indicator of absolute disparity for unordered group data because it weights by population group size and is sensitive to the magnitude of larger deviations from the

Figure 14. Disparity in Mammography Screening Among Racial/Ethnic Groups, NHIS, 2000

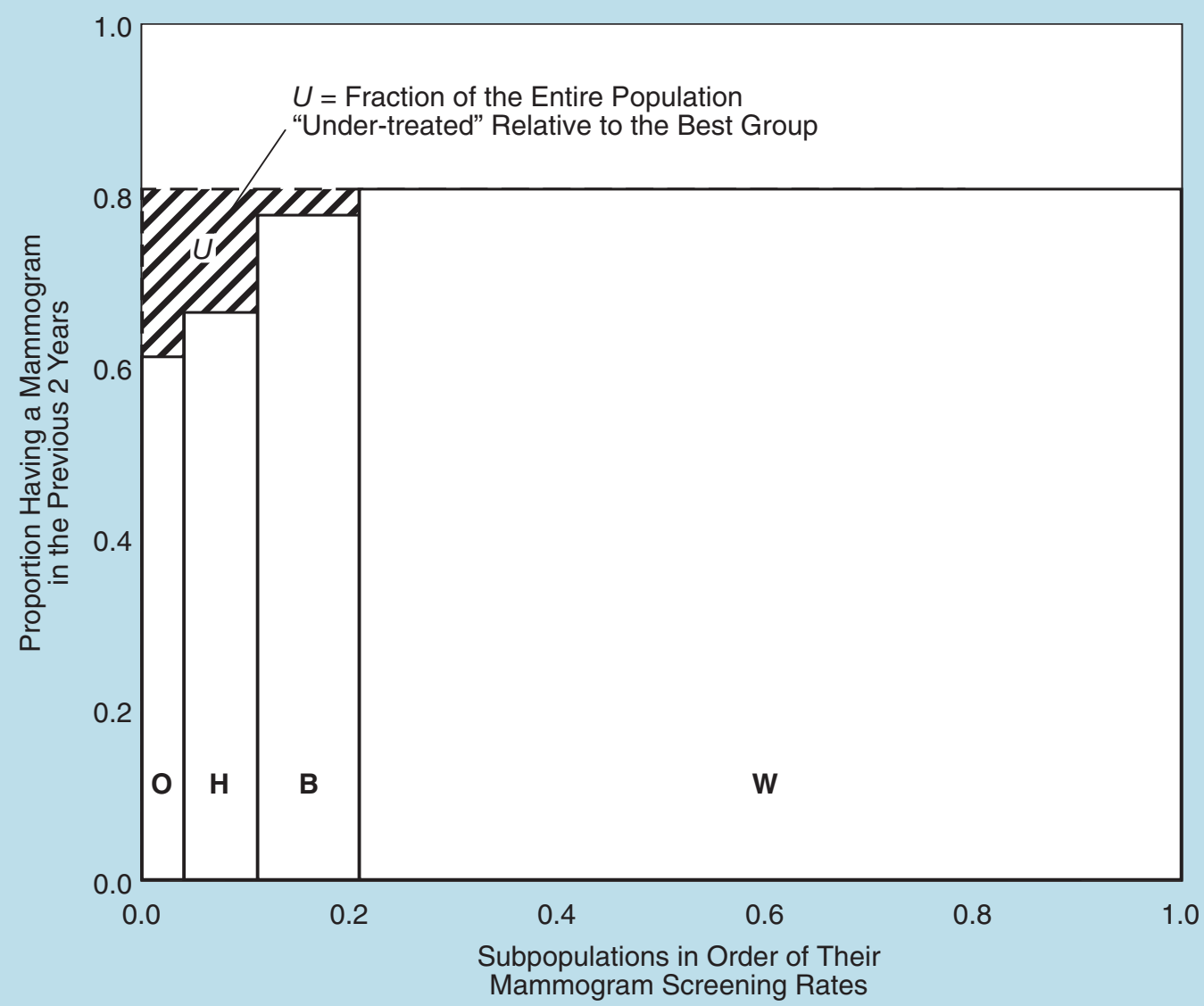

Source: Gastwirth JL. Prev Med (forthcoming). 
population average. As an example, Figure 15 shows trends in age-adjusted lung cancer mortality among U.S. Census divisions.

The between-region variance in lung cancer mortality in 1968 was 7.1 deaths per 100,000, but in 1998 the $B G V$ was 22.8 deaths per 100,000.

This larger absolute disparity in regional mortality indicates divergent regional trends in lung cancer over time (see Figure 15). The use of the variance as a measure of disparity in economics sometimes is discouraged because it is not "scale invariant." In other words, it is sensitive to absolute changes, such as when everyone's income doubles over time. In this case, economists sometimes feel that it is not desirable for the disparity measure also to double, because relative inequality is maintained. Although this may be an undesirable property when dealing with income disparity, however, we believe it is not necessarily a limitation for discussing health disparity, in which we are interested in absolute disparity burdens (84). From a population health perspective, in which we may be concerned with the health care implications of increasing absolute disparity, we may care about situations in which the absolute disparity increases, and it is appropriate that the disparity indicator reflect this increased concern. In this case, then, using the variance (which squares the absolute deviations from the population average)

\section{Figure 15. Age-Adjusted Lung Cancer Mortality by U.S. Census Division, 1968-1998}

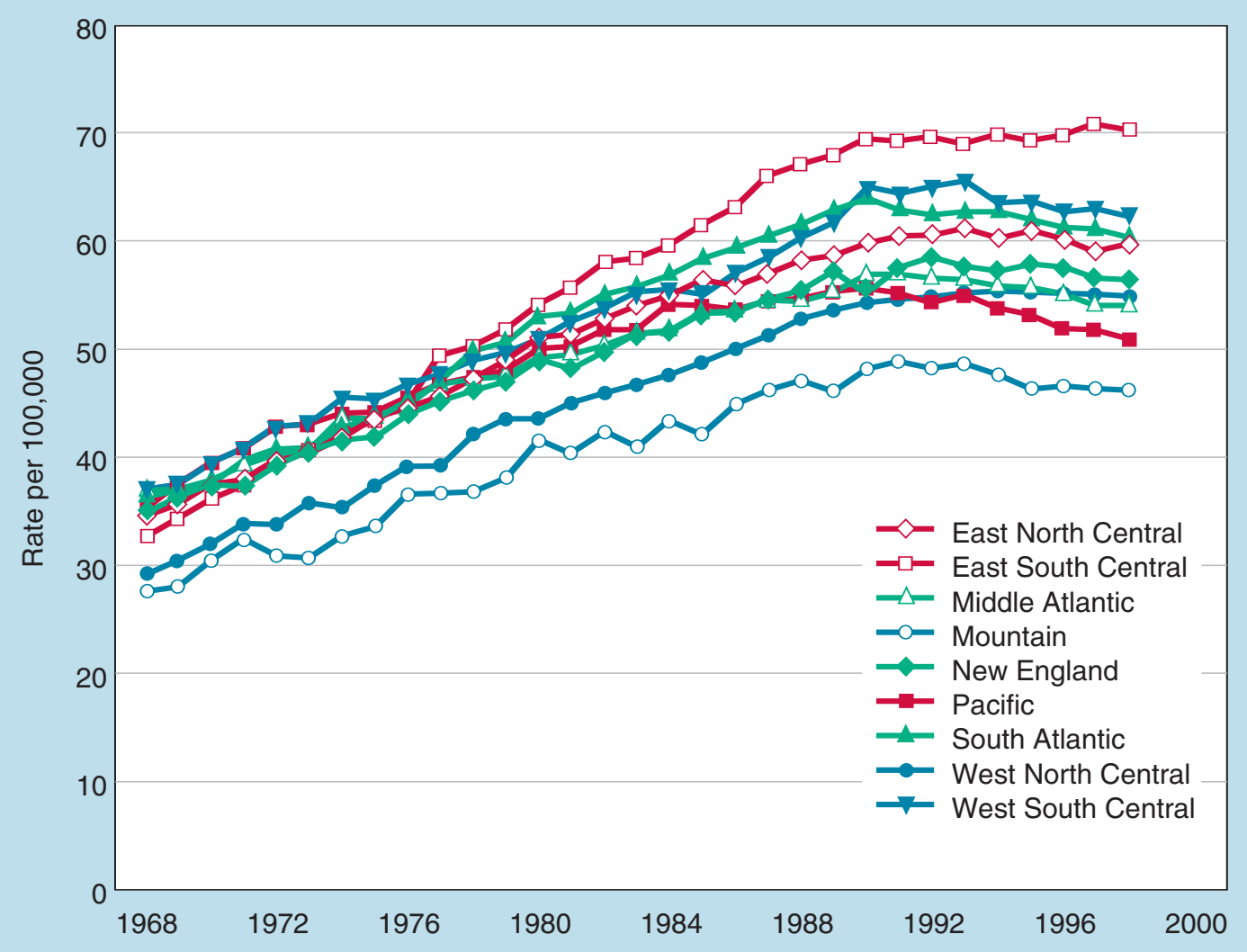

Source: NCHS. Compressed Mortality Files 1968-1998. 
is consistent with a population health perspective. In the example above of changes in regional lung cancer disparity, the overall rates increase by $70 \%$, the coefficient of variation (the variance divided by the mean) increases by $89 \%$ (indicating that relative disparity increases as well), but the variance increases by $320 \%$. Thus, choosing this as the measure of disparity reflects our concerns with widening absolute differences among the regions.

\section{Measures of Average Disproportionality}

When describing health inequalities, public health researchers and policy makers often use what might be called the "language of disproportionality." For example, in the context of arguing for the importance of measuring health inequalities between socially meaningful population groups, Braveman and colleagues stated that "a disproportionate share of ill-health and premature mortality is borne by the socially disadvantaged" (51, page 233). Similarly, in discussing health disparity trends, the Secretary of Health and Human Services recently noted that various racial/ethnic groups in the United States "suffer an unequal burden of death and disease, despite improvements in the overall health of the general population over the past decade" (85). The terms "disproportionate share" and "unequal burden" are important descriptors because they communicate the ethical notions inherent in the collective concerns over health disparities. That is, they capture the notion that it is unfair that some groups experience more ill health than others; a just distribution of health implies that ill health should be experienced proportionately by different social groups. A more explicit example can be found in the Guidance for the U.S. National Healthcare Disparities Report in which, in discussing the disparity in cardiac catheterization rates between blacks and whites, LaVeist states that the "degree to which the predicted percentage of catheterization deviates from the observed percentage indicates the degree of disparity," and concludes that "African Americans received 67\% of the catheterizations that they should have received, and whites received 14\% more than their share" (86, page 90).

The quotations above make clear that health disparity often is equated with the concept of disproportionality. What is perhaps less clear is that, in the context of the commonly used "language of disproportionality," there usually is an implied reference group, which is the general population. In fact, in the catheterization example, LaVeist was arguing explicitly against measuring health disparity using a relative measure such as a risk ratio or odds ratio, because doing so means using a particular social group (in this case, whites) as the reference group, which necessarily assumes that the rate in the reference group is "most desirable." Thus, he argued that disparity measures that use whites as the reference group would not be able to identify their "overutilization" of cardiac catheterization. The intuitive ethical notion expressed in the quotations above is that the amount of ill health in social group $j$ is far greater than would be expected if ill health were evenly distributed with respect to all $J$ social groups. An even distribution of ill health across $J$ social groups implies that the 
number of individuals of social group $j$ with condition $y$ is proportionate to group $j$ 's share of the total population, so that the rate of ill health, $Y_{i}$ in each of the $j$ groups is exactly the same, which would necessarily equal the rate in the total population. Thus, the proportional distribution of $y$ among $J$ groups implies that $Y_{i}=\bar{Y}$ (the mean of $y$ ) for all groups.

This is an important point because many commonly used measures of income disparity (e.g., the Gini coefficient) and residential segregation (e.g., the Index of Dissimilarity), some of which currently are employed to measure health disparities, may be expressed conveniently as measures of average disproportionality (87-89). For each social group $j$, we can define a health (or ill health) ratio as the ratio of measure $y$ in the $j$ th group to that of the mean of $y$ for the whole population, so that $r_{j}=Y_{j} / \bar{Y}$ for each group. Note that this makes such measures relative rather than absolute disparity indicators. In this framework, measures of disparity take the general form

$$
I=\sum_{j} p_{j} f\left(r_{j}\right)
$$

where $p_{j}$ is group $j$ 's proportion of the total population and $f\left(r_{i}\right)$ is some disproportionality function of the ratio $r_{j}=Y_{j} / \bar{Y}$. It should be clear that equation [18] is a weighted disparity measure because each group's disproportionality function $f\left(r_{j}\right)$ is multiplied by its population share $p_{\vec{l}}$ Measures of this type of disparity indicator differ because they implement different disproportionality functions. Perhaps one of the appealing features of such measures is that they provide a rather direct correspondence between the commonly used languages of health disparity in terms of "disproportionality" with the operationalization of the measurement.

Figure 16 (page 49) depicts the concept of "disproportionality" using data on all deaths in the United States, by gender and education, for the year 2000. Among males, those with less than 12 years of education bear a disproportionate burden of all deaths, as they account for $24 \%$ of all male deaths but account for only $12 \%$ of the male population. Conversely, males with greater than 12 years of education account for $55 \%$ of the total population but only $32 \%$ of all deaths. The level of disproportionality for females with less than 12 years of education is slightly smaller.

Table 2 (page 49) shows some commonly used statistical measures and their disproportionality functions. Readers should note that the measures differ only in how they express the difference between shares of health and shares of population.

\section{Entropy Indices}

One class of disproportionality measures that often is favored by economists are measures of general entropy, developed by Henri Theil (90). The example described below is for measuring the disparity in BMI, which is a risk factor for a number of cancer sites $(91,92)$. Theil's index gives relatively more weight to the concentration in the upper end of the health distribution and is calculated (with grouped data) by summing the product of each group's BMI share of the population's total BMI and the natural log of each 
Figure 16. Example of the "Disproportionality" of Deaths and Population, by Gender and Education, 2000

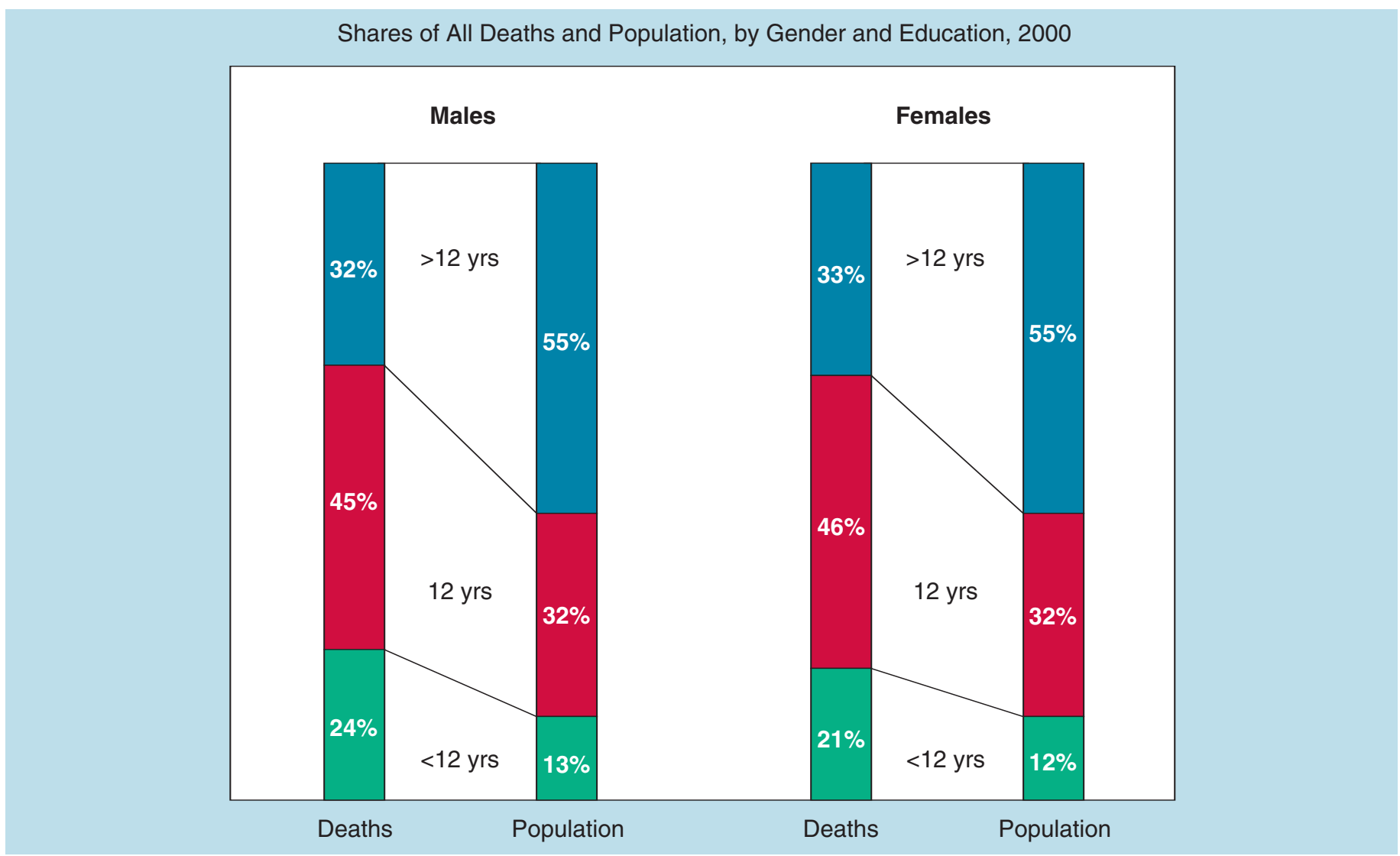

Source: NCHS. Deaths: Final Data for 2000, Natl Vit Stat Rep 2002;50(15).

\section{Table 2. Commonly Used Disproportionality Functions}

\begin{tabular}{|c|c|}
\hline Squared coefficient of variation $\left(C V^{2}\right)$ & $\left(r_{j}-1\right)^{2}$ \\
\hline Gini index $(G)$ & Individual-level data: $\left|r_{i}-r_{j}\right| / 2$ \\
\hline Relative concentration index $(\mathrm{RCl})$ & $\begin{array}{l}\text { Same as for } G \text {, but groups are ranked by social group position instead of by health, so that } q_{j} \text { is the proportion of } \\
\text { the total population in groups less advantaged than group } j \text {, and } Q_{j} \text { is the proportion of the total population in } \\
\text { groups more advantaged than group } j \text { (i.e., } p_{j}+q_{j}+Q_{j}=1 \text { ) }\end{array}$ \\
\hline Mean logarithmic deviation (MLD) & $\ln \left(1 / r_{j}\right)=-\ln \left(r_{j}\right)$ \\
\hline Variance of log-health (VarLog) & {$\left[\ln r_{j}-\Sigma\left(\ln r_{j}\right)\right]^{2}$} \\
\hline
\end{tabular}

Note: Adapted from Firebaugh, 2003 (88). 
group's BMI share. For individual-level data, total disparity in BMI measured by Theil's index can be written as

$$
T=\sum_{i=1}^{N} p_{i} r_{i} \ln \left(r_{i}\right)
$$

where $p_{i}$ is an individual's population share (which in the case of individual data will be $1 / n$, so that $\sum p_{i}=1$ ) and $r_{i}$ is the ratio of the individual's BMI to the population average BMI (i.e., $r_{i}=y_{i} / \bar{Y}$ ). When the population of individuals is arranged into $J$ groups, Theil showed that equation [19] is the exact sum of two parts: between-group disparity and a weighted average of within-group disparity:

$T=\sum_{j=1}^{J} p_{j} r_{j} \ln \left(r_{j}\right)+\sum_{j=1}^{J} p_{j} r_{j} T_{j}$

where $T_{j}$ is the disparity in BMI within group $j$. The within-group component (the second term on the right side of equation [20] is weighted by, in this case, group j's share of the total BMI, because $p_{j} \times r_{j}=s_{j}$ (where $s_{j}$ is the share of total BMI) when the denominator for $r_{j}$ is the mean BMI for the total population. More importantly, the above decomposition also makes it clear that it is possible to calculate between-group disparity in BMI-the primary quantity of interest with respect to social disparities in health-in the absence of data on each individual. The only data needed are the group proportions and the ratio of the group's BMI to the population average BMI. Between-group disparity, however, may increase because total disparity is increasing (i.e., both between-group and within-group disparity are increasing simultaneously). The primary advantage of using additively decomposable inequality measures is that they allow us to determine not just whether between-group disparity is increasing, but whether the share of total disparity that is due to disparity between groups is increasing or decreasing. Although this measure has attractive qualities, the betweengroup/within-group decomposition requires continuous outcome data measurable in individuals, so it is not clear whether this can be applied to many relevant cancer outcomes that are based on events (e.g., incidence, mortality, or screening). Even for noncontinuous outcomes, however, entropy indices easily can be used to calculate between-group disparities in the absence of individual-level data. For example, suppose that instead of BMI we wanted to measure the between-group disparity in obesity rates. We could do this by calculating the first term on the right side of equation [20] using only the data on each group's proportion in the population $\left(p_{j}\right)$ and the group's rate of obesity relative to the overall population rate $\left(r_{j}\right)$-data that are more likely to be readily available.

Measuring between-group inequality in BMI in the above manner makes clear that changes in the value of disparity over time are a function of two quantities: changing group proportions and changing social group BMI ratios. This is important- in the case of obesity, for examplebecause differentiating between these two components of change has different implications for obesity as a public health problem and may be the result of very different social policies. If we find that disparity is increasing but that the main reason for the observed change is that the share of the population among social groups at the tails of the BMI distribution has increased, it simply demonstrates that the inequality increase is due primarily to the movement into and out of 
different social groups—not to differentially increasing rates of BMI within subgroups of a social group. However, if we find that population shares have remained relatively constant over time but BMI disparity has increased because BMI ratios are increasing, this implicates differential sources of BMI change among particular groupswhich may then become the target of public health intervention.

\section{Atkinson's Measure}

Atkinson's index actually is not a single index of disparity but depends on specifying the relative sensitivity of the index to different parts of the distribution. One way of writing Atkinson's index is:

$A=1-\left[\sum_{j=1}^{J} p_{j} r_{j}^{1-8}\right]^{1 /[1-\varepsilon}, \mathcal{E}>0$

where $p_{j}$ and $r_{j}$ are again, respectively, the share of population and the health ratio (relative to the total population rate), as defined above. Clearly with this index, the extent of disparity hinges on specifying the parameter $\varepsilon$, which indicates the degree of "aversion to disparity." Larger values of $\varepsilon$ indicate stronger aversion to disparity, which also may be interpreted as placing increased weight on the least healthy groups. For example, if we are particularly concerned about improving the health of least-healthy individuals, we could make the measure of disparity more sensitive to changes in the bottom of the health distribution.

\section{Gini Coefficient}

The Gini coefficient summarizes social group differences in, for example, BMI for the entire population and can be thought of as a measure of association between each social group's share of population, ranked by the group's health and its share of health. Its formula for individual data is given above for the IID in equation [2], when $\alpha=2$ and $\beta=1$. Formally, the Gini coefficient is the ratio of the area between the line of equality in Figure 17 (page 52) and the Lorenz curve to the total area of the triangle beneath the line of equality (40). Because the Gini coefficient is a function of the disproportionality between shares of population and shares of health, one can see from Figure 17 that health disparity increases as the Lorenz curve moves further away from the line of equality (i.e., as the disproportionality between shares of population and shares of health increases).

\section{Concentration Index}

The Concentration Index $(C I)$ is calculated similarly to the Gini index, but it results from a bivariate distribution of health and social-group ranking. In the same way that the Gini coefficient is derived from the Lorenz curve, the $C I$ is derived from a concentration curve, where the population is ordered first by social-group status (rather than by health status, as for the Gini), and the cumulative percent of the population then is plotted against the group's share of total ill health. When the y-axis is the share of ill health, this results in the Relative Concentration Index $(R C I)$; however, an Absolute Concentration Index $(A C I)$ also may be derived by plotting the cumulative share of the population against the cumulative amount of ill health (i.e., the cumulative contribution of each subgroup to the mean level of health in the population). Figure 18 (page 53) gives a graphical representation of a relative concentration curve. Note the similarity with the Lorenz curve drawn in Figure 17 to illustrate the Gini coefficient. The two curves and 
Figure 17. Representation of the Gini Coefficient of Disparity

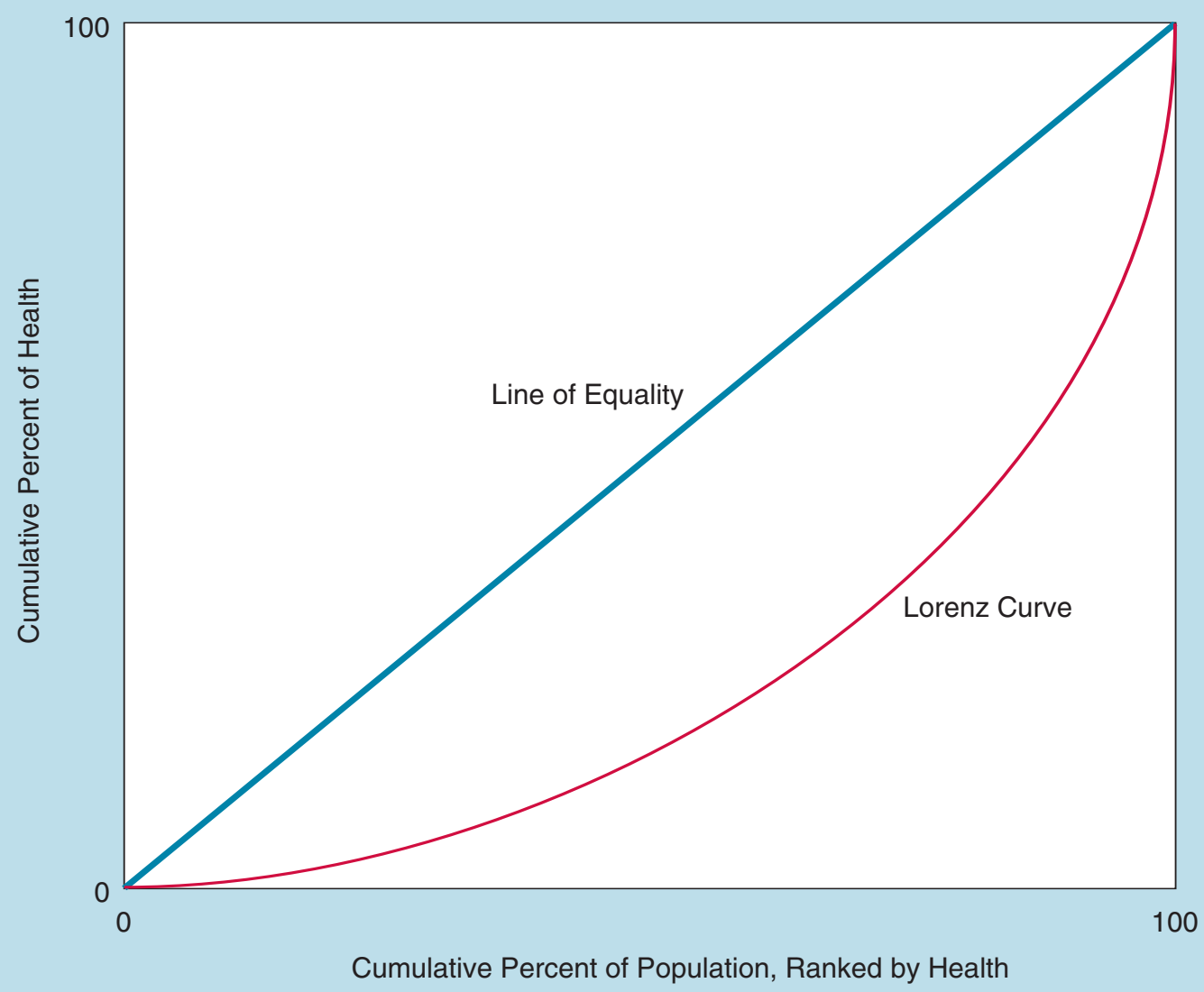

thus the Gini coefficient and the $R C I$ are calculated similarly, the only difference being the way in which social groups are ordered. In the case of the Gini coefficient, social groups are ordered by their health status (lowest to highest), regardless of their socioeconomic group ranking; for the $R C I$, social groups are ordered by their ranking in terms of, for example, years of education, regardless of their health status. It is important to note that, because the Concentration Index incorporates information on both health and social-group status, the concentration curve may lie either above or below the line of equality. The general formula for the Relative Concentration Index $(R C I)$ for grouped data is given by Kakwani and colleagues (93) as:

$R C I=\frac{2}{\mu}\left[\sum_{i=1}^{J} p_{j} \mu_{j} R_{i}\right]-1$

where $p_{j}$ is the group's population share, $\mu_{j}$ is the group's mean health, and $R_{j}$ is the relative rank of the $j$ th socioeconomic group, which is defined as:

$R_{j}=\sum_{j=1}^{J} p_{\gamma^{-}} \frac{1}{2} p_{j}$

where $p_{\gamma}$ is the cumulative share of the population up to and including group $j$, and $p_{j}$ is the share of the population in group $j$. $R_{j}$ essentially indicates the cumulative share of the population up to the midpoint of each group interval, similar to the categorization of the Slope and Relative Index of 
Inequality above. In fact, the $R C I$ has a specific mathematical relationship with the RII (60):

$R C I=2 \operatorname{var}(R) R I I$

where $R$ is the relative rank variable identified in equation [23]. Thus, the SII/RII and the $A C I / R C I$ will produce the same rank ordering of health inequality over time but will differ in scale. The absolute version of the Concentration Index $(A C I)$ is calculated by multiplying the $R C I$ by the mean rate of the health variable:

$A C I=\mu R C I$

where $\mu$ is the mean level of health in the population. It also is worth pointing out that, when using continuous health outcomes, the RCI is unbounded and takes minimum and maximum values of -1 and +1 , but when using binary health outcomes, the possible values of the $R C I$ are limited by the mean (e.g., the prevalence) of the distribution (94). Adam Wagstaff shows that, for a given nonzero mean of a binary variable $(\mu)$, the minimum of the $R C I$ is $[\mu-1+(1 / n)]$ and the maximum is $[1-\mu+(1 / n)]$, with $n$ being the sample size. This of course has implications for analyses that compare the extent of socioeconomic inequality in health between areas or outcomes with very different levels of average health, and one potential strategy for facilitating comparisons is to normalize the Concentration Index by dividing it by its bound (95).

\section{Figure 18. Representation of the Health Concentration Curve}

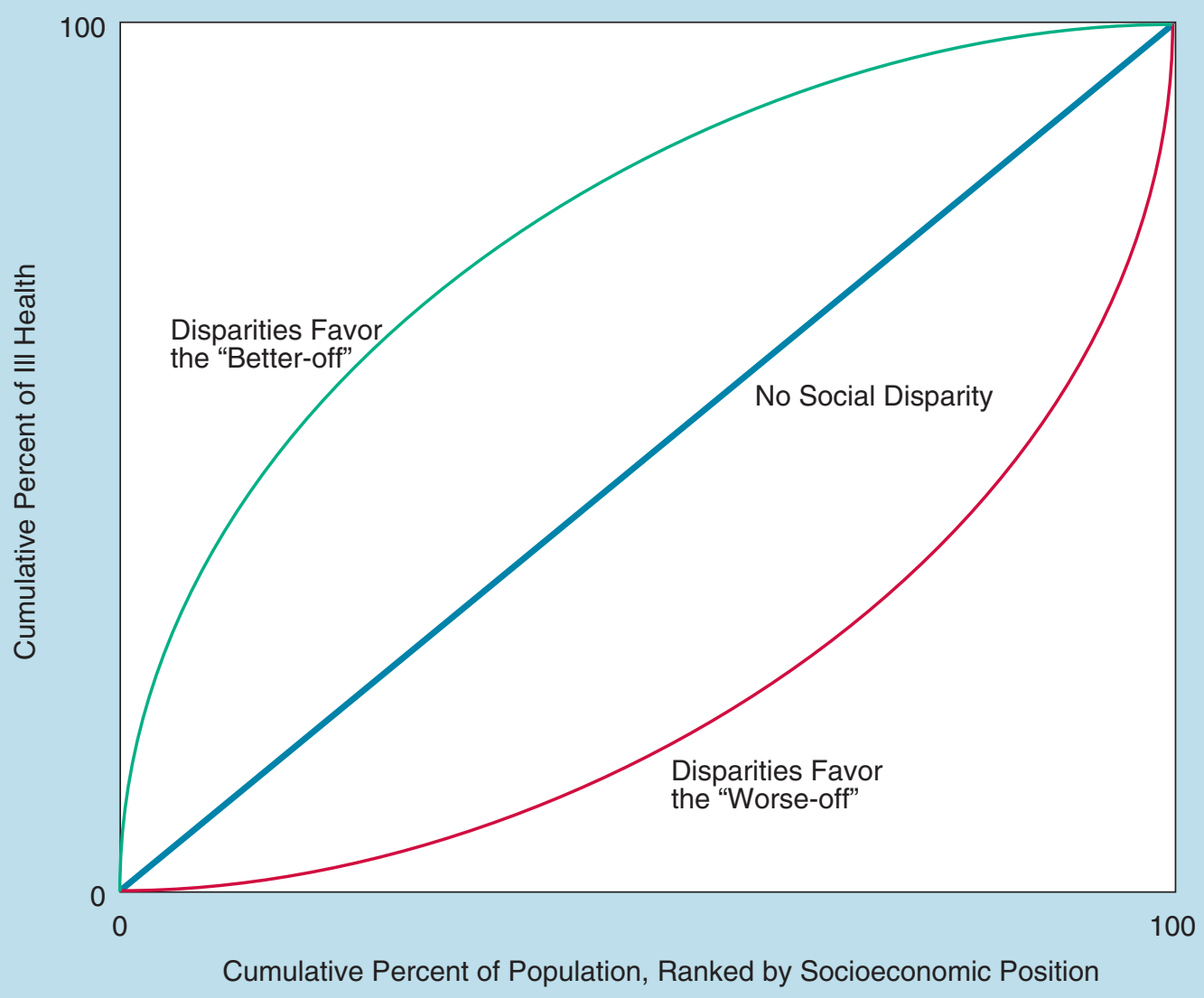




\section{Table 3. Educational Disparity in Lung Cancer Mortality, 1999}

\begin{tabular}{|c|c|c|c|c|}
\hline Education & Rate per 100,000 & Population Share & Midpoint (\%) & $\mathrm{Cl}$ \\
\hline$<12$ years & 49.8 & 0.128 & 0.064 & 0.408 \\
\hline 12 years & 41.0 & 0.327 & 0.292 & 3.913 \\
\hline$>12$ years & 16.9 & 0.545 & 0.728 & 6.699 \\
\hline \multirow[t]{3}{*}{ T otal } & 29.0 & 1.0 & & 11.020 \\
\hline & & Relative Concentration Index & $\rightarrow$ & -0.240 \\
\hline & & Absolute Concentration Index & $\rightarrow$ & -6.959 \\
\hline
\end{tabular}

Note: Rates are for persons aged 25-64 years and exclude the following states: Georgia, Kentucky, Rhode Island, and South Dakota. Rate data are from DATA2010...the Healthy People 2010 Database, April 2004 edition, and SEER ${ }^{\star}$ Stat. Population data are from NCHS, Deaths: Final Data for 1999: Table VII.

Table 3 shows a simple example of how the $R C I$ and $A C I$ are calculated using equations [22] and [25] with grouped data using lung cancer mortality rates by educational attainment. One can see that lung cancer mortality rates decrease with increasing education, and the negative value of both indices shows that the disparity in lung cancer mortality favors the better educated.

One of the reasons the $A C I$ and $R C I$ (and, by extension, the $S I I / R I I$ indices) are favored by some is that they "reflect the socioeconomic dimension to inequalities in health" (60, page 548). That is, a downward health gradient (such that health worsens with increasing social-group rank) results in a positive index, whereas an upward health gradient results in a negative index. For example, if the data in Table 3 were reversed so that lung cancer mortality rates for those with $<12$ years of education were 16.9 and the rates were 49.8 for those with $>12$ years of education, the $R C I$ then would be calculated as 0.114 and the $A C I$ as 4.873, indicating that lung cancer mortality actually favors the less educated. This sensitivity to the direction of the health gradient is not a property of other disproportionality measures, such as the Gini coefficient and the Index of
Dissimilarity, because they do not depend on the strict ordering of social groups.

This undoubtedly is an advantage of the $R C I$, but, as with all disparity measures, it also may be seen as a disadvantage. Because of its sensitivity to socioeconomic gradients in health, the $R C I$ may not register any disparity when health is not ranked directly by social group. Thus, when a social group ranked in the middle of a hierarchy bears a disproportionate burden of ill health, the $R C I$ well may register this as zero disparity. This is not just a theoretical limitation of the RCI. For instance, age-adjusted rates of breast cancer deaths (per 100,000) in the United States in 1998 were 20.0 among those with less than a high-school education, 28.4 among those with a high-school education, and 22.0 among those with at least some college education (9). If the respective shares of the population in each of the education groups were approximately $38.8 \%, 20 \%$, and $41.2 \%$, the $R C I$ would be virtually zero, indicating no educational mortality disparity; yet those with a high-school education will contribute roughly $40.3 \%$ of breast cancer deaths. A reasonable case could be made that a disproportionate burden of breast cancer falls on the high-school-educated 
(using this categorization of education), but the $R C I$ would not reveal this pattern. This pattern of the worst health among those in the middle social group is not simply an artifact of breast cancer as an unusual cause of death. This pattern also is seen for colorectal and prostate cancers and melanoma as well where rates across ordered social groups are not simple gradients.

We use the breast cancer example not necessarily to suggest that the $R C I$ is a poor index for measuring social-group disparities in health, but rather to emphasize that all disparity measures have advantages and disadvantages that should be considered when selecting and interpreting a disparity index; no summary disparity measure should be used as a substitute for detailed inspection of the health status indicators for each social group via tables and graphs.

Wagstaff also derived a method for incorporating a society's degree of aversion to disparity into the $R C I$, which he calls the "extended" Concentration Index (96). The aversion parameter changes the weight attached to the health of different socioeconomic groups in a manner similar to the Atkinson Index described above. The formula for this extended version of the $R C I$ for grouped data is:

$$
R C I(v)=v \sum_{j=1}^{J} p_{j}\left(1-R_{j}\right)^{v-1}-\frac{v}{\mu} \sum_{j=1}^{J} p_{j} \mu_{j}\left(1-R_{j}\right)^{v-1}[26]
$$

where $v$ is the "aversion parameter," and the other quantities are defined as in the $R C I$ in equation [22] above. Setting $v=1$ weights every group's health equally (i.e., complete indifference to inequality), and setting $v=2$ gives the standard $R C I$ defined above. As $v$ increases, the weight attached to the health of lower socioeconomic groups increases, and the weight attached to the health of higher socioeconomic groups decreases. Table 4 (page 56) shows the effect of varying the weight placed on the health of the less-educated groups for the disparity in current smoking in the state of Michigan in 1990 and 2002. The two rightmost columns are the calculated $R C I s$, with differing aversions to disparity. Setting $v=2$ gives the standard $R C I$ of -0.129 , indicating that the disparity in current smoking favors the better educated. Increasing the weight placed on the health of the less-educated groups in 1990 results in only a marginal increase in the measure of disparity to -0.178 , most likely because the rate of smoking among those with $<8$ years of education actually is quite low. The major effect of the differential weighting of the $R C I$ can be seen in the disparity change from 1990 to 2002; in 2002, the standard $R C I(2)$ was -0.19 , a disparity increase of $48.5 \%$, and the more bottom-sensitive $R C I(4)$ was -0.32 , indicating a much larger $81 \%$ increase in the relative education disparity in smoking. The reason the increase in disparity is so much larger for $R C I(4)$ is that, although rates of smoking decreased overall and in every other education category, the estimated rate of current smoking actually increased among the least-educated group. This example shows how we can incorporate an ethical judgment (particular concern about the health status of the least educated) into a measure of health disparity. 


\section{Table 4. Example of Extended Relative and Absolute Concentration Index Applied to the Change in Educational Disparity in Current Smoking, Michigan, 1990 and 2002}

\begin{tabular}{|c|c|c|c|c|c|}
\hline Education & Population (\%) & $\%$ smokers & Midpoint (\%) & $R C I\left(v={ }_{2}\right)$ & $R C l\left(v={ }_{4}\right)$ \\
\hline \multicolumn{6}{|l|}{1990} \\
\hline$<8$ years & 4.5 & 27.2 & 2.3 & 0.006 & 0.011 \\
\hline 12 years & 36.9 & 33.3 & 35.4 & -0.068 & -0.057 \\
\hline $13-15$ years & 27.3 & 29.6 & 67.5 & -0.003 & -0.001 \\
\hline $16+$ years & 18.9 & 13.6 & 90.6 & 0.019 & 0.000 \\
\hline T otal & 100.0 & 29.1 & & $\begin{array}{c}-0.129 \\
A C l=-3.75\end{array}$ & $A C l=-5.16$ \\
\hline \multicolumn{6}{|l|}{2002} \\
\hline$<8$ years & 2.0 & 36.6 & 1.0 & -0.021 & -0.041 \\
\hline $9-11$ years & 7.8 & 36.3 & 5.9 & -0.073 & -0.130 \\
\hline 12 years & 31.4 & 31.3 & 25.5 & -0.137 & -0.152 \\
\hline \multirow[t]{2}{*}{ T otal } & 100.0 & 24.2 & & -0.191 & -0.321 \\
\hline & & & & $A C l=-4.63$ & $A C l=-7.77$ \\
\hline
\end{tabular}

Note: Data is for current smoking and is drawn from the 1990 and 2002 Behavioral Risk Factor Surveillance System (BRFSS).

\section{Combining Health Disparity and Average} Health

As we emphasized in the above discussion of relative and absolute health disparities, the goals of Healthy People 2010 are couched specifically in terms of both health disparities and average levels of population health. Thus, we also may be interested in investigating potential ways to incorporate both average health and health disparity into a single summary measure. One potential measure, created by Adam Wagstaff, is called the Health Achievement Index or HAI (96).

The Health Achievement Index

The HAI in some respects is similar to the $A C I$ described above, but combines disparity and average health by essentially subtracting the Absolute Concentration Index from the population's average health, creating a "disparitydiscounted" level of average health. The formula for the HAI is (96):

$$
\begin{aligned}
H A I(v) & =\mu\lfloor 1-R C I(v)\rfloor \\
& =\mu-A C I(v)
\end{aligned}
$$

where $\mu$ is the population's average health and $R C I(v)$ is the extended Relative Concentration Index defined above. Thus, applying equation [27] to the data on the average rate and educational disparity (using $R C I[2])$ in smoking in Michigan, the $\operatorname{HAI}(2)$ for Michigan in 1990 is $0.29 \times(1-[-0.129])=0.33$, and is $0.24 \times$ $(1-[-0.191])=0.29$ in 2002. Clearly, if $R C I=0$, then the $H A I$ is equal to the population average rate of health, and the larger the $R C I$, the further away the $H A I$ is from the population average-a kind of "disparity penalty" applied to the population average rate. In this sense, the HAI is 
not exactly a measure of health disparity but a potentially useful way of capturing both aggregative (Healthy People 2010 Goal 1) and disparity (Healthy People 2010 Goal 2) concerns in a single summary measure, at least with respect to ordered social groups. For example, while both relative and absolute education disparity in smoking increased in Michigan over the 12-year period (i.e., education disparity unambiguously increased, see Table 4), health achievement actually improved because almost all education groups experienced a decrease in the rate of smoking (in this case, health "achievement" increases as the population rate of smoking decreases).

Two populations (or time periods) therefore might have the same value on the Achievement Index but differ greatly on both average health and the extent of health disparity. For example, in Figure 19 (page 58) the relative concentration curves for education disparity in obesity in New York State are plotted for 1990 and 2002. Because the 2002 curve is beneath the 1990 curve for every education group, we can unambiguously declare that relative education inequality in obesity in New York decreased from 1990 to 2002. The standard RCIs summarizing the two curves also reflect the decrease in disparity, going from -0.284 in 1990 to -0.125 in 2002. As was mentioned at the outset, however, we do not believe that disparity is all that matters. We also are concerned with the average health of the population, and the estimated obesity rate in New York more than doubled over this period, from $9.8 \%$ in 1990 to $20.6 \%$ in 2002. Figure 20 (page 59) shows the absolute concentration curves, which clearly reflect the increase in obesity among all groups. Inspection of the education-group-specific obesity rates reveals that, in general, the education disparity in obesity declined because of increasing obesity rates, particularly among the middle- and better-educated groups. When we incorporate the adverse changes in overall population rates of obesity with the changes in disparity, the change in the Health Achievement Index (for which larger values are worse because the health outcome, obesity, is negative) indicates that things became worse, having increased from 0.13 in 1990 to 0.23 in 2002. 
Figure 19. Relative Concentration Curves for Educational Disparity in Obesity in New York State, BRFSS, 1990 and 2002

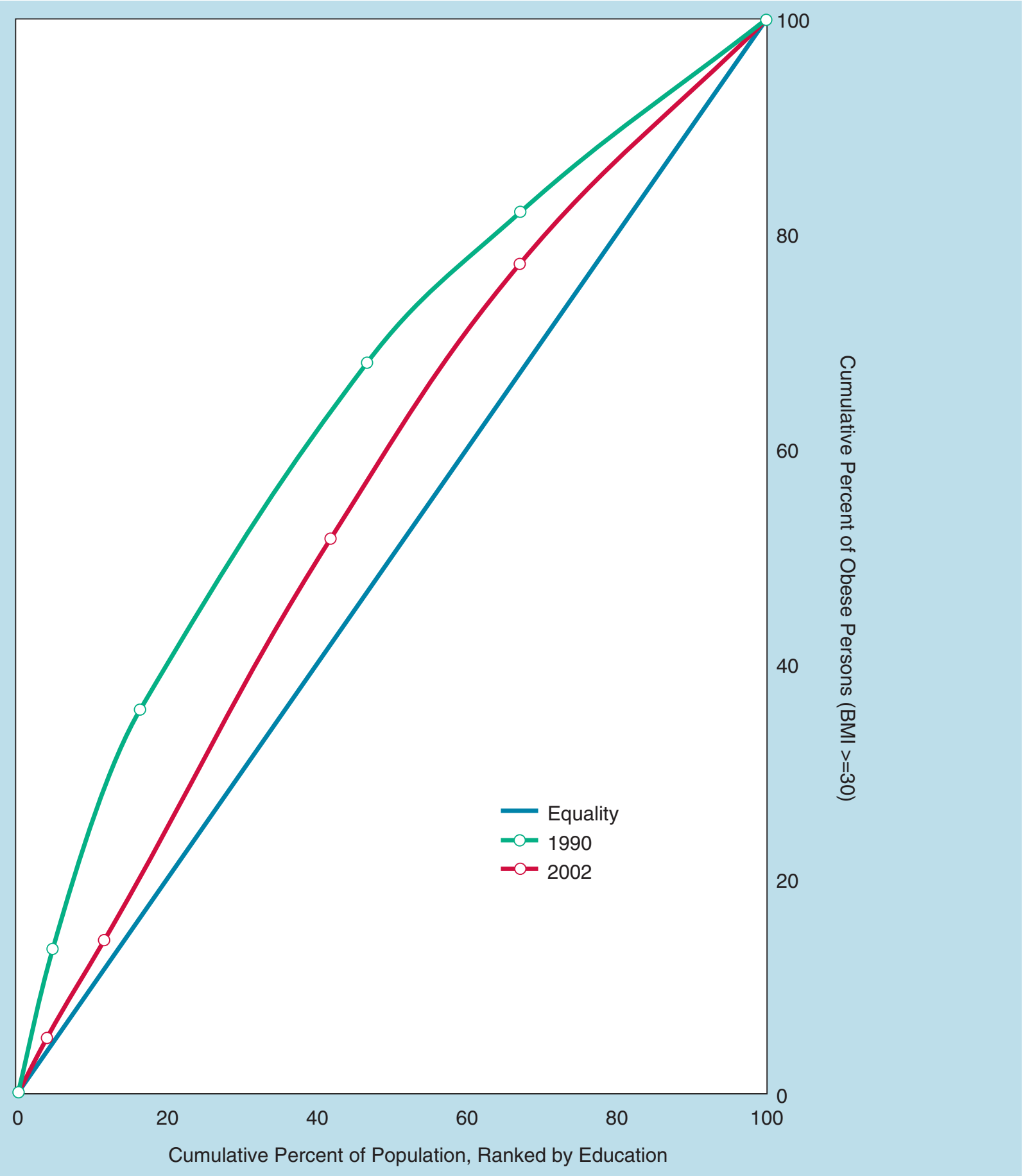


Figure 20. Absolute Concentration Curves for Educational Disparity in Obesity in New York State, BRFSS, 1990 and 2002

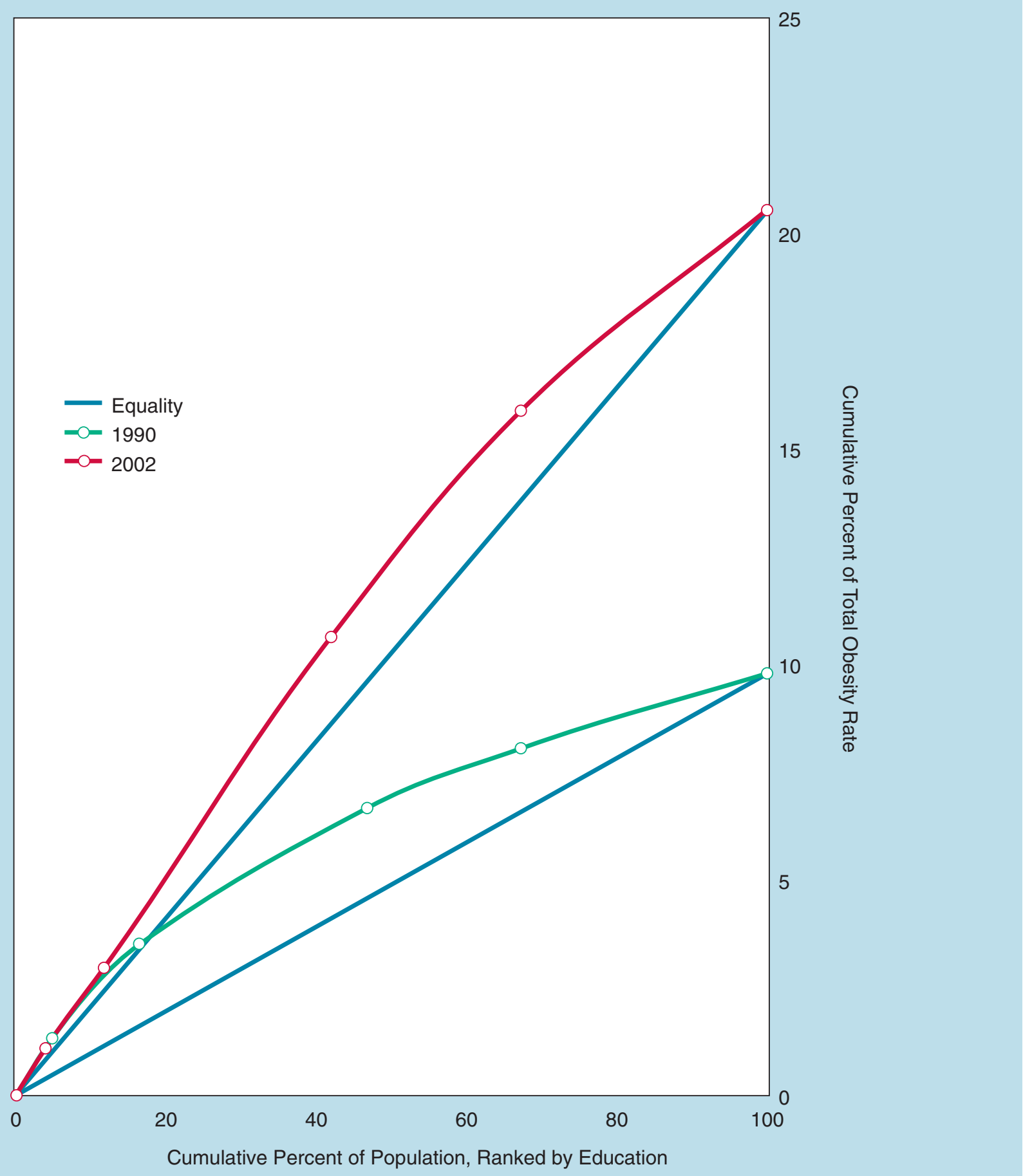




\section{Choosing a Suite of Health Disparity Indicators}

Monitoring progress toward the elimination of disparities in cancer-related health objectives involves a number of ethical, conceptual, and methodological issues that must be given careful consideration to answer the question of which measure or measures should be employed to monitor progress toward the elimination of health disparities between social groups.

One possibly useful way to approach the measurement of health disparity is to consider a sequence of methodological approaches.

- First, we cannot emphasize enough the close inspection of the underlying subgroup-specific health outcomes (rate or prevalence, etc), either via tabular or graphical inspection. This is likely to reveal important population health patterns, highlight the situation of specific subgroups of interest, and lend an understanding of any underlying heterogeneity that a summary measure of health disparity may not emphasize.

- Next, consider the relevant question that is to be answered. If one is interested in the health disparity between two particular groups-for example, the trend in the disparity between black and white males in lung cancer mortality-then the use of a pairwise comparison of trends is sufficient.

- Even for assessing health-disparity trends between two groups using a pairwise comparison, we recommend using both an absolute and a relative disparity measure. This is especially warranted when making long-term comparisons that may involve steep declines or increases for all social groups. Although the relative disparity measure gives some indication of the progress (or lack thereof) one group is making, regardless of the actual level of health, absolute disparity measures provide a context in which to judge the public health impact of relative health disparities. Thus, we would argue for the primacy of the absolute indicator of disparity. Efforts to improve public health often rely on the absolute burden of disease; thus, the absolute disproportionality in health disparity also should have priority. This is the case especially when comparing the size of social-group disparity across different cancer outcomes and risk factors. We also understand, however, that this in no way excludes particular cases in which the relative inequality may be judged - for other equally good reasons- to be of high priority despite low levels of disproportionate absolute burden. For instance, the twofold relative disparity in cervical cancer between black and white women may be judged to be especially important because it is a health disparity that is almost entirely avoidable through the routine use of screening, even though the absolute disparity involves only about 5 deaths per 100,000 .

- If one is interested in monitoring the health disparity trend across the entire range of 
subgroups within a social-group category-for example, across all racial/ethnic groups rather than comparing, say, blacks and whites-or if the social-group category has many subgroups-such as the disparity across the 50 U.S. states-then summary measures of health disparity are warranted. The first decision involved in choosing a summary measure is dictated by whether or not the social groups in question have a natural ordering.

\section{Summary Indicators}

\section{Ordered Social Groups}

If the social group does have a natural ordering, as with education and income groups, then we recommend using either the Absolute Concentration Index $(A C I)$ or Slope Index of Inequality (SII) as a measure of absolute health disparity, and the Relative Concentration Index $(R C I)$ or the Relative Index of Inequality $(R I I)$ as a measure of relative disparity. The major reasons for choosing these particular measures are that

- they account for changes in the underlying population distributions in the social groups over time and use information across the entire range of social groups;

- they are flexible enough to allow different levels of aversion to disparity to be incorporated; and

- they are sensitive to the direction of the social gradient in health.
Although this last criterion mainly is what distinguishes these measures from other summary measures of inequality, such as the Gini coefficient or the Index of Dissimilarity, we would also reemphasize that, if the social groups in the middle of the distribution (e.g., those with a highschool education as opposed to those with less or more education) experience a disproportionate burden of ill health, our selected measures may indicate that no disparity exists when it in fact could be argued otherwise. Of course, if the sequence laid out above is adhered to, then Step 1 -a simple and careful inspection of the basic subgroup data—should reveal this.

This is part of the "cost" of using summary measures of disparity, but in this case a comparison of the $R C I$ or RII with another measure of disproportionality that is not strictly sensitive to health gradients, such as the Gini coefficient or Theil index, may reveal important information about the social distribution of health. Lastly, because the RCI has a mathematical relationship to the RII, and the $A C I$ has such a relationship to the SII, they always will result in the same rank ordering of health distributions. That being said, one additional desirable feature of the $A C I$ and $R C I$ is the ability to graph their associated health concentration curves. Although the ability of any summary measure of health disparity to communicate important information about disparity trends to health policy makers may be limited, the $A C I$ and $R C I$ may serve this purpose better than the RII and SII. 


\section{Unordered Social Groups}

Our recommendations for health disparity measures for ordered groups restate the recommendations of earlier reviews of health disparity measures $(60,70)$. In the context of the Healthy People 2010 goals, however, groups with a natural ordering represent only a small number of the social groups across which we want to eliminate disparities in cancer-related health outcomes. Therefore, we also need to think about disparity measures that can be applied to unordered social groups. Again, we would emphasize choosing a summary measure of health disparity only when one is interested in monitoring the extent of disparity across more than two or three social groups. For comparisons of two specific groups, there is no substitute for simple pairwise measures of absolute and relative disparity.

If comparisons across multiple unordered groups are needed, we recommend the BetweenGroup Variance $(B G V)$ as a summary of absolute disparity and the general entropy class of measures developed by Henri Theil as summary measures of relative disparity (more specifically, the Theil index and the Mean Log Deviation). An important reason for choosing the Between-Group Variance and the entropy-based measures is that they are disparity measures that can be decomposed perfectly into between-social-group and within-social-group components, given a continuous health outcome. This cannot be said of other measures, such as the Gini coefficient and Atkinson's measure $(97,98)$. The ability of the variance and the entropy class of disparity measures to decompose disparity is important because it allows one to look at any number of cross-classified social groups, whether ordered or not. For example, race and income, or gender and education, can be examined jointly to assess the trend in disparity between certain dimensions of society. We could look first, for example, at the trend in between-race disparity in cancer survival time and see whether the disparity is increasing or decreasing. We then could look at the disparity between race-education groups over time. It may be that, while the between-race disparity is decreasing, educational disparities within race groups actually are increasing. In addition, the entropy-based measures also can be decomposed to investigate the relative effects of changing social group distributions versus changing health distributions. This is important because both of these aspects of the population constantly are changing over time. Understanding the relative impact of health changes versus compositional changes in social groups is important for understanding the prospects for intervening to eliminate health disparities. Thus, because of their decomposition properties, the entropy measures may be useful tools for describing and understanding the stratification of cancer-related health outcomes across time.

Our recommendation for measures of health disparity, for both ordered and unordered social groups, come from explicitly adopting the population health perspective toward monitoring health disparities. By taking a population health perspective, we emphasize using the total population as the reference group for measuring health disparity, weighting social groups according to the number of individuals they represent, and examining both absolute and relative disparities in health. By doing so, we are able to account for changes in the distribution of 
individuals across social groups over time, a fact that has clear population health consequences, and we also are able to express health disparities in a way that emphasizes their overall burden on population health.

\section{Table 5. Summary Table of Advantages and Disadvantages of Potential Health Disparity Measures}

\begin{tabular}{|c|c|c|c|c|c|c|c|c|}
\hline Disparity Measure & Symbol & $\begin{array}{l}\text { Absolute } \\
\text { or } \\
\text { Relative }\end{array}$ & $\begin{array}{l}\text { Reference } \\
\text { Group }\end{array}$ & $\begin{array}{r}\text { All } \\
\text { Social } \\
\text { Groups }\end{array}$ & $\begin{array}{r}\text { Reflect } \\
\text { SES } \\
\text { Gradient }\end{array}$ & $\begin{array}{c}\text { Social } \\
\text { Group } \\
\text { W eighting }\end{array}$ & $\begin{array}{l}\text { Inequality } \\
\text { A version } \\
\text { Parameter }\end{array}$ & $\begin{array}{l}\text { Graphical } \\
\text { Analogue }\end{array}$ \\
\hline \multicolumn{9}{|l|}{ T otal Disparity } \\
\hline Inter-Individual Difference & $I I D$ & Variable & ATBO $^{a}$ & No & No & No & Yes & No \\
\hline Individual-Mean Difference & $I M D$ & Variable & Average & No & No & No & Yes & No \\
\hline \multicolumn{9}{|l|}{ Social Group Disparity } \\
\hline Absolute Difference & $A D$ & Absolute & Best & No & Yes & No & No & Yes \\
\hline Relative Difference & $R D$ & Relative & Best & No & Yes & No & No & Yes \\
\hline Regression-Based Relative Effect & $R R E$ & Relative & Best & Yes & Yes & $\mathrm{No}^{\mathrm{b}}$ & No & Yes \\
\hline Regression-Based Absolute Effect & $R A E$ & Absolute & Best & Yes & Yes & $\mathrm{No}^{\mathrm{b}}$ & No & Yes \\
\hline Slope Index of Inequality & SII & Absolute & Average & Yes & Yes & Yes & No & Yes \\
\hline Relative Index of Inequality & RII & Relative & Average & Yes & Yes & Yes & No & Yes \\
\hline Index of Disparity & $I D_{\text {isp }}$ & Relative & Best & Yes & No & No & No & No \\
\hline Population Attributable Risk & $P A R$ & Absolute & Best & Yes & No & Yes & No & Yes \\
\hline Population Attributable Risk\% & $P A R \%$ & Relative & Best & Yes & No & Yes & No & No \\
\hline Index of Dissimilarity & $I D$ & Absolute & Average & Yes & No & Yes & No & Yes \\
\hline Index of Dissimilarity\% & $I D \%$ & Relative & Average & Yes & No & Yes & No & No \\
\hline Relative Concentration Index & $\mathrm{RCl}$ & Relative & Average & Yes & Yes & Yes & Yes & Yes \\
\hline Absolute Concentration Index & $\mathrm{ACl}$ & Absolute & Average & Yes & Yes & Yes & Yes & Yes \\
\hline Between-Group Variance & $B G V$ & Absolute & Average & Yes & No & Yes & Yes & No \\
\hline Squared Coefficient of Variation & $C V^{2}$ & Relative & Average & Yes & No & Yes & No & No \\
\hline Atkinson's Measure & $A$ & Relative & Average & Yes & No & Yes & Yes & No \\
\hline Gini Coefficient & Gini & Relative & Average & Yes & No & Yes & No & Yes \\
\hline Theil Index & $T$ & Relative & Average & Yes & No & Yes & Yes & No \\
\hline Mean Log Deviation & $M L D$ & Relative & Average & Yes & No & Yes & Yes & No \\
\hline Variance of Logarithms & Varlog & Relative & Average & Yes & No & Yes & No & No \\
\hline
\end{tabular}




\section{Appendix: Example Analyses}

This appendix presents two examples of how to measure and monitor cancer-related disparity trends using the suite of indicators and measurement strategy outlined in the recommendations above. The examples are taken from the cancer-related objectives outlined in Healthy People 2010 and are illustrative rather than substantive analyses. The first example assesses the trend in the prevalence of mammography screening among education groups and emphasizes disparity measures for ordered social groups. The second example assesses the trend in colorectal cancer mortality among racial groups and highlights the use of disparity measures for unordered social groups.

\section{Example 1: Educational Disparity in Mammography Screening, 1990-2002}

This example is based on self-reported data on the use of mammography screening from the Centers for Disease Control and Prevention's (CDC) Behavioral Risk Factor Surveillance Survey (BRFSS), an annual state-based telephone survey of adults. Although perhaps not the optimal source of data on routine cancer screening because of its high rate of nonresponse, the BRFSS may be used to approximate a nationally representative sample (99), and its annual administration and timely reporting of results make it useful for illustrating disparity trends.

\section{Method}

- Step 1: Inspect the underlying subgroup data. Figure A1 (page 66) shows the prevalence of women over age 40 who reported having not received a mammogram in the past 2 years for five education groups. From the chart, two things are immediately clear. First, all groups are improving over time (i.e., the proportion of women not having mammograms is declining), and some groups have actually achieved the Healthy People 2010 target rate of $30 \%$. Second, there is a graded relationship between the proportion of women not having a mammogram in the past 2 years and years of educationwomen with fewer years of education are less likely to have received a mammogram in the past 2 years.

- Step 2: Determine the disparity question to be answered. For this example, we are concerned about whether the extent of disparity in mammography screening across all education groups is increasing or decreasing. As a result, we will use a summary measure of health disparity. Suppose, however, that there had been a dedicated effort to increase the rate of screening among women with a high-school education. We then might be more interested in the question of whether the effort had narrowed the gap in screening between women with the highest 
Figure A1. Proportion of Women Age 40 and Over Who Did Not Receive a Mammogram in the Past 2 Years by Educational Attainment, 1990-2002

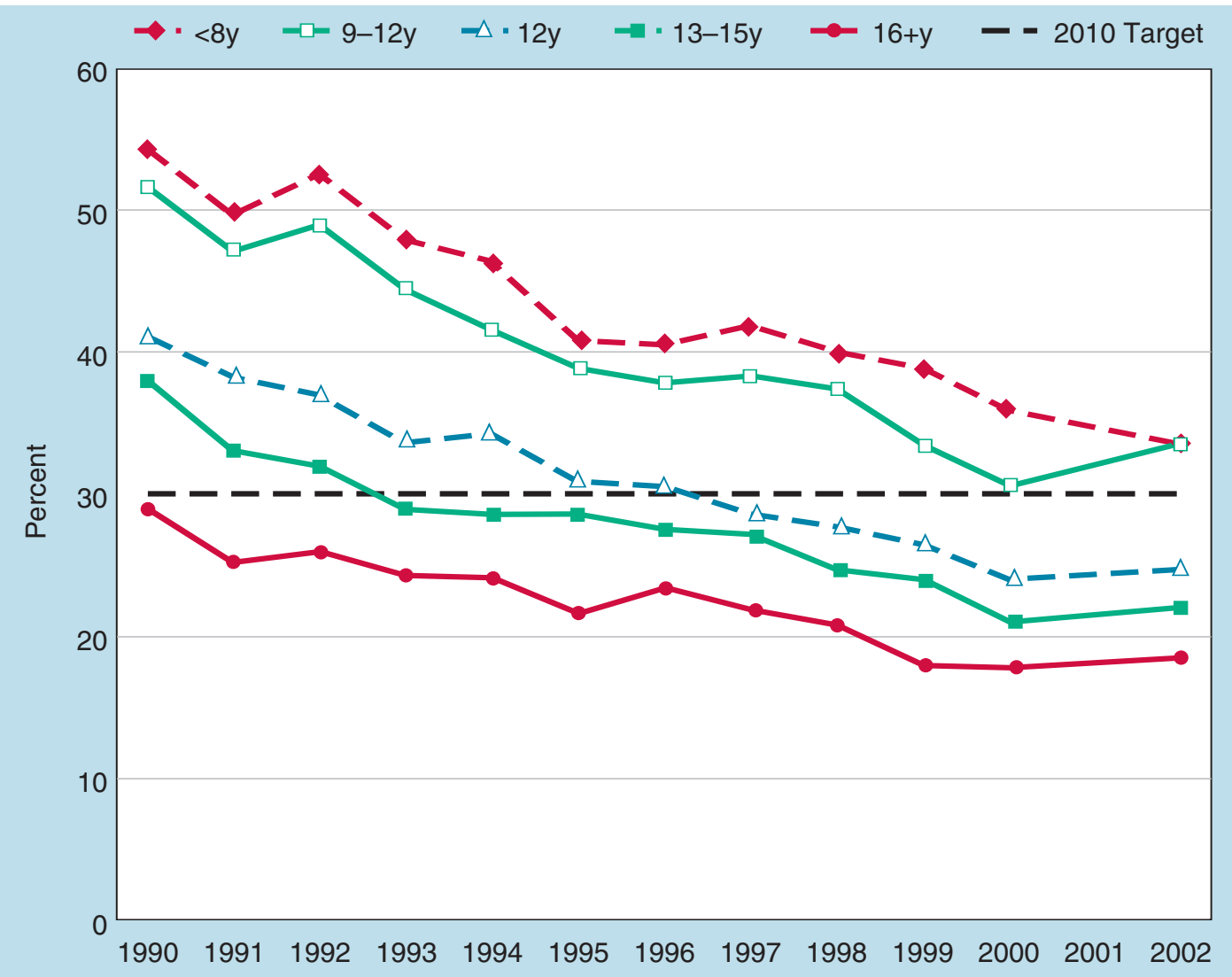

Source: CDC, Behavioral Risk Factor Surveillance Surveys 1990-2002.

screening rates (college-educated) and highschool-educated women. In this case, a summary measure might mask an important change in this particular subgroup, so we would use a simple pairwise comparison of the absolute and relative difference in screening.

- Step 3: Choose a summary measure of health disparity. Having decided to use a summary measure, the choice of a measurement tool hinges on whether the social groups in question have a natural ordering. In the case of years of education, there clearly is a natural ordering, so we will choose the Relative Concentration Index
$(R C I)$ as a measure of relative disparity and the Absolute Concentration Index $(A C I)$ as a measure of absolute disparity. Both measures are calculated for two levels of disparity aversion, $v=2$, which gives the standard $R C I$ and $A C I$ (96), and $v=4$, which gives additional weight to the health of the lower-educated groups.

\section{Results}

For the beginning and end of the period 1990-2002, Table A1 (page 68) shows the prevalence of not having a mammogram in the past 2 years $\left(\mu_{i}\right)$ and the population proportion 
$\left(p_{j}\right)$ for each group. The relative ranking of each interval $\left(R_{\mathrm{j}}\right)$, based on the midpoint of the cumulative population distribution, is derived by applying equation [23] above; thus, for the group with 9-11 years of education, the relative ranking is $0.09+(0.5 \times 0.14)=0.17$. This particular group's contribution to the $R C I(2)$ is calculated using equation [26] above as [ $2 \times 0.14 \times$ $\left.(1-0.17)^{(2-1)}\right]-[(2 / 41.0) \times 51.6 \times 0.14 \times$ $\left.(1-0.17)^{(2-1)}\right]=-0.0618$. Applying equation [26] across all education groups gives the overall $R C I(2)$, which for $1990=-0.1045$. To give this value some perspective, Zhang and Wang, using income as a measure of socioeconomic position and using data from the National Health and Nutrition Examination Survey (NHANES III) (100), report a relative Concentration Index of -0.055 for obesity. Because in this case the outcome is the proportion of women not having a mammogram, the negative sign of $R C I(2)$ indicates that the disparity favors better-educated women. A positive $R C I(2)$ in this case would have indicated that disparity actually favors less-educated women. Additionally, the effect of increasing the aversion to disparity becomes readily apparent as the value of the $R C I$ in 1990 increases (from -0.10 to -0.19 ) when the aversion to disparity increases twofold. Our preferred measure of absolute disparity for ordered social groups, the Absolute Concentration Index $(A C I)$, also is displayed in Table $\mathrm{A} 1$ and is calculated from equation [27] above; for 1990 $A C I(2)=41.0 \times(-0.1045)=-4.2$.

Comparing 1990 and 2002 it appears that, as measured by the standard $A C I$ (i.e., with $v=2$ ), absolute disparity in mammography screening declined substantially over those 12 years-a $43.9 \%$ reduction. Relative disparity showed only slight improvement, with the $\mathrm{HCI}(2)$ declining only $2.6 \%$. Note that, with an increased aversion to disparity $(v=4)$, relative disparity actually shows a slight increase (from -0.191 to -0.201 ), while absolute disparity still declines (from -7.8 to -4.7). Although this indicates virtually no change in relative disparity, Figure A2 (page 69) shows that, when calculated annually, relative disparity was not consistent over the entire period. As the prevalence of not having a mammogram fell faster for the less-educated groups in the first half of the 1990s, both absolute and relative disparity declined (i.e., the best possible scenario). As screening rates tapered off among the lesseducated groups, however, relative disparity increased in the latter half of the 1990s, eventually returning to the 1990 level by 2002 . Note also that two components of the $R C I$ and $A C I$ were changing over the period of observation: the prevalence rates and the population shares in each education group. Most notably, the share of the population with less than 12 years of education-the groups with the highest prevalence rates-declined from 24\% (0.09 among those with $<8$ years plus 0.14 for those with $9-11$ years) to $14 \%(0.06+0.08)$. Because the $R C I$ is a population-weighted index, this change alone (i.e., in the absence of any change in prevalence rates) would serve to decrease the level of disparity. Thus, a logical next step in analyzing the disparity trend might be to decompose the change in disparity to determine how much of the change is due to declining prevalence rates and how much is due to upward shifting of the education distribution. We will not go through the decomposition steps here, but we would reemphasize that such decomposition techniques are likely to be useful in understanding the sources of changes in health disparities. 
Table A1. Example of Relative and Absolute Concentration Index Applied to the Change in Educational Disparity in Mammography, 1990 and 2002

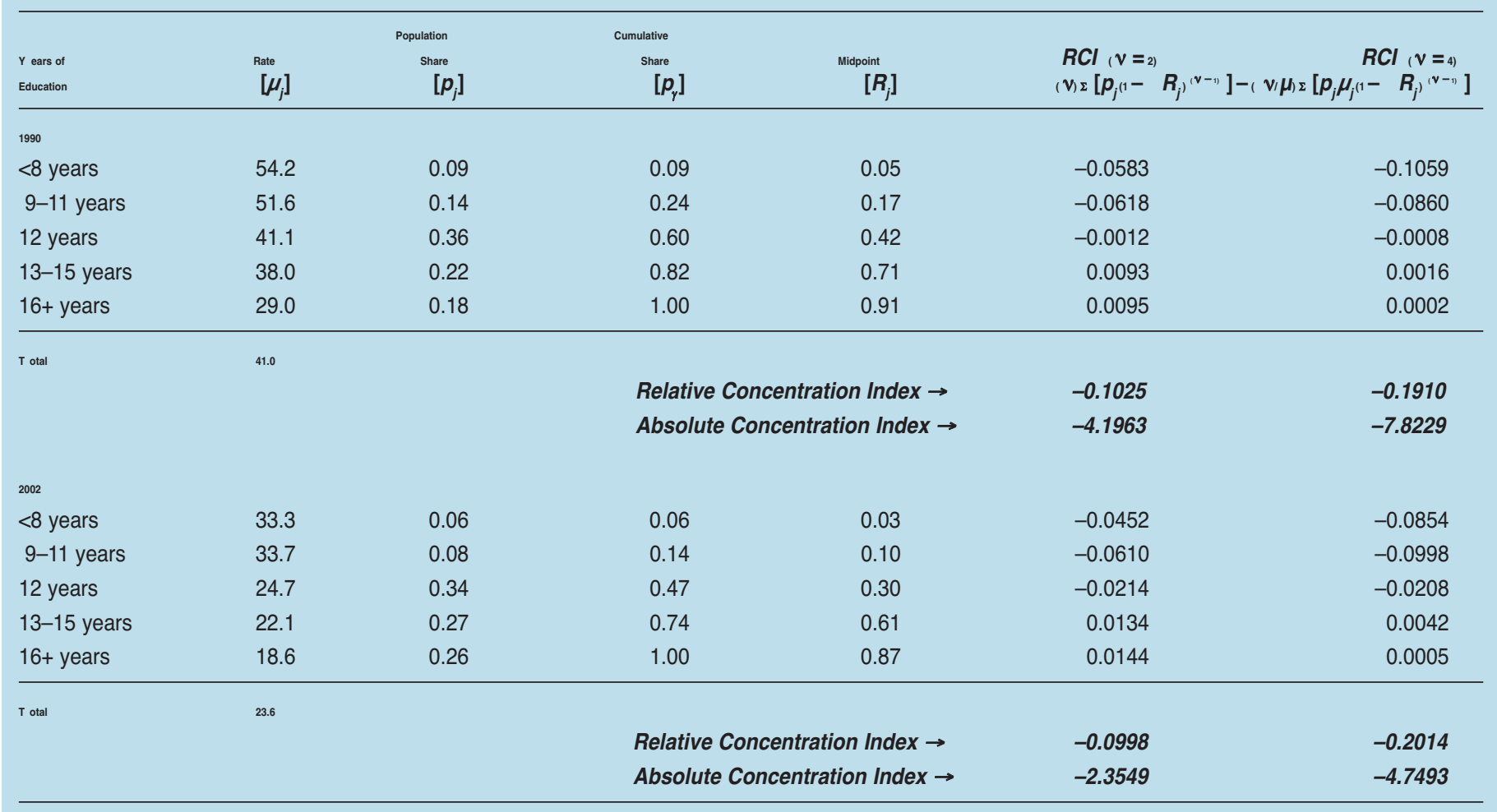

Note: Data is for the proportion of women over 40 who did not have a mammogram within the past 2 years and is drawn from the 1990-2002 Behavioral Risk Factor Surveillance System.

Thus, by using two summary measures of disparity, we can make some generalization about the trend in the education-related disparity in mammography screening. Between 1990 and 2002, the relative disparity between education groups remained essentially the same, but the absolute disparity declined because the prevalence of not having a mammogram declined in all education groups, with the largest absolute declines having occurred among the less-educated groups. Thus, we would argue that the population health burden associated with the educationrelated disparity in mammography screening has decreased. This, of course, is not the best possible scenario, but we would argue that the disparity situation is better in 2002 than it was in 1990 .

Lastly, we would re-emphasize that our conclusion about the socioeconomic disparity trend in mammography reflects a population health perspective on health disparities. Because we were concerned about the disparity across all education groups, we chose two populationweighted summary disparity measures. The results obtained by the application of these measures may or may not be consistent with other disparity measures that reflect concern about a different dimension of disparity. 
Figure A2. Trends in Education-Related Disparity and Prevalence for the Proportion of Women Age 40 and Over Who Did Not Receive a Mammogram in the Past 2 Years, 1990-2002

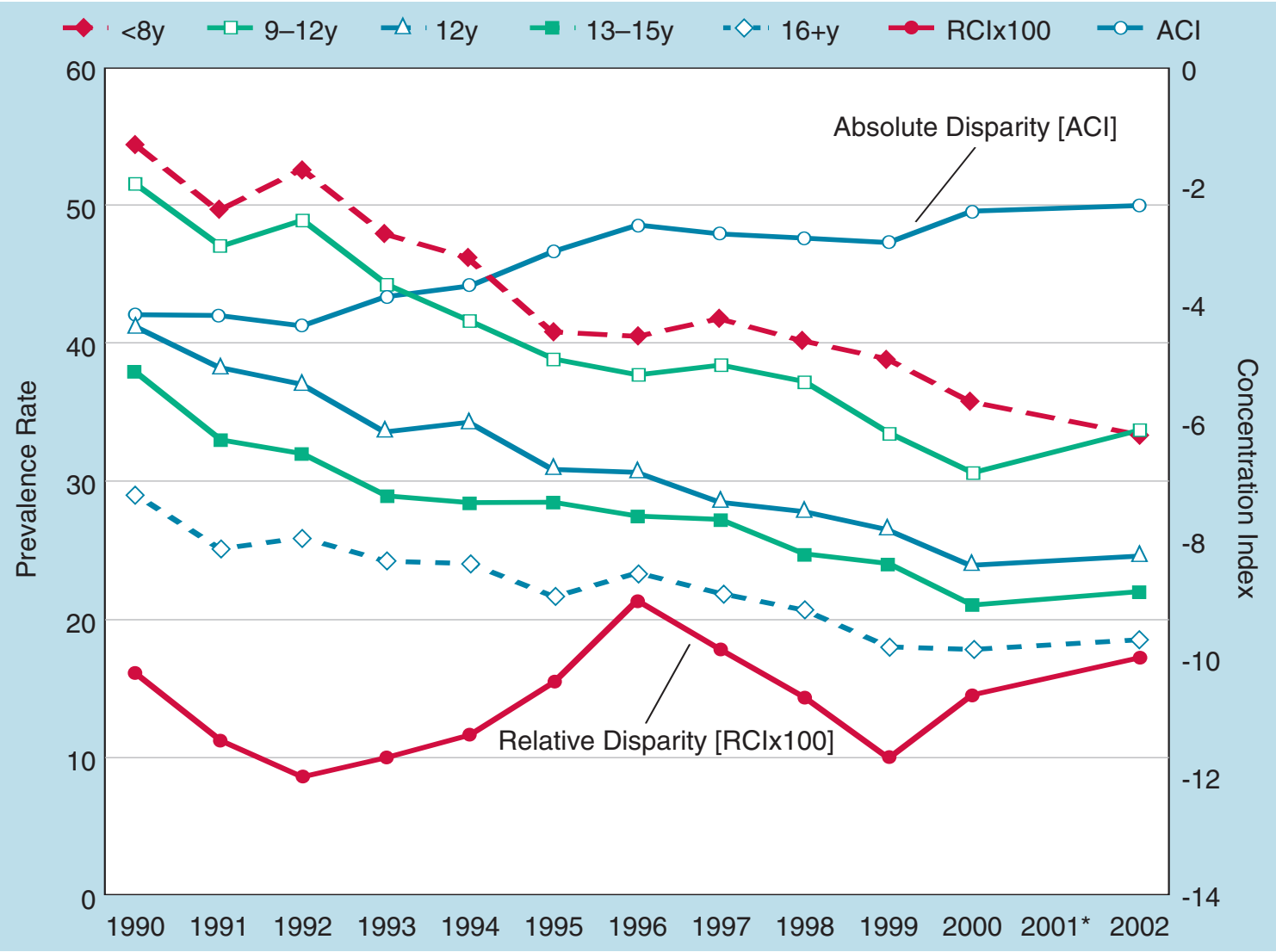

Source: CDC, Behavioral Risk Factor Surveillance Surveys 1990-2002.

*Note: Question not asked in 2001.

\section{Example 2: Racial Disparity in Colorectal Cancer Mortality, 1990-2002}

Our second example concerns rates of colorectal cancer mortality from 1990 to 2002 among four racial groups: American Indian/Alaska Natives, Asian/Pacific Islanders, blacks, and whites. The data for this example come from NCI's SEER Program (www.seer.cancer.gov). There are several limitations to this example that should be recognized. First, it is not possible with current data to analyze longer trends for mutually exclusive detailed racial/ethnic groups because many states did not have complete information on ethnicity in death certificate data. Second, there is great heterogeneity of cancer risk among subgroups of American Indian/Alaska Natives and Asian/Pacific Islanders that is not reflected in this simple example because we are forced to combine these groups. Similar issues of lack of homogeneity arise in comparing groups defined by ethnicity and by various social, economic, and geographic factors. 


\section{Method}

- Step 1: Inspect the underlying subgroup data. Figure A3 (page 71) shows trends among those aged 45-64 years for colorectal cancer mortality for the four racial groups. Blacks have the highest rates of colorectal cancer mortality, followed by whites, and then by American Indian/Alaska Natives and Asian/Pacific Islanders, both of whom have similar mortality rates that actually are near the 2010 age-adjusted target rate. Both blacks and whites experienced relatively steady declines in colorectal cancer mortality rates during the 1990s (although the decline in black rates seems to slow down after the mid-1990s), but the rates among other groups remained somewhat steady, hovering near the 2010 target rate.

- Step 2: Determine the disparity question to be answered. Although a number of interesting pairwise comparisons could be made, in the spirit of the Healthy People 2010 goals we are interested in the extent to which progress is being made toward the elimination of racial disparities in colon cancer mortality. This would be achieved when all groups have the same mortality rate, so we will use a summary measure of health disparity to determine if racial disparities in working-age colorectal cancer mortality are increasing or decreasing.

- Step 3: Choose a summary measure of health disparity. Clearly, there is no natural ordering among racial groups, so having decided to use a summary measure of health disparity, we will use the Between-Group Variance ( $B G V$ ) to measure the absolute level of disparity and two entropybased measures of relative disparity—-the Theil index (T) and the Mean Log Deviation (MLD). Although it is not necessary to use both $T$ and $M L D$ to measure relative health disparity, it may be instructive to do so for reasons outlined below.

\section{Results}

Table A2 (page 72) shows the colorectal cancer mortality rate $\left(\mu_{j}\right)$, the rate relative to the population average rate (i.e., the mortality ratio $\left.r_{i}\right)$, and the population share $\left(p_{j}\right)$ for each racial group in 1990 and 2001. Recall that both $T$ and $M L D$ are measures of average disproportionalitythat is, they take the general form $\sum_{i} p_{j} f\left(r_{j}\right)$ and measure the extent to which shares of population and shares of health differ-but they use different disproportionality functions (i.e., different specifications of $\left.f\left(r_{j}\right)\right)$. The disproportionality function for $T$ is $r_{j} \ln \left(r_{j}\right)$ and for $M L D$ is $-\ln \left(r_{j}\right)$ or, equivalently, $\ln \left(1 / r_{j}\right)$. It may not seem immediately clear why it might be helpful to use both measures, but it may be more clear if we use some simple algebra to rewrite the equations. First, note that when the denominator for the mortality ratio $r_{j}$ is the total population rate, $r_{j} \times p_{j}=s_{j}$, where $s_{j}$ is the share of mortality for racial group $j$. We can then rewrite the disproportionality function $f\left(r_{j}\right)$ for $T$ as $\frac{s_{j}}{p_{j}} \ln \left(\frac{s_{i}}{p_{i}}\right)$ and MLD as $1 \mathrm{n}\left(\frac{p_{j}}{s_{i}}\right)$. When we apply the general formula $\sum_{i} p_{j} f\left(r_{j}\right)$ for measures of average disproportionality, the formula for $T$ becomes $\sum s_{i} 1 \mathrm{n}\left(\frac{s_{j}}{p_{j}}\right)$ and MLD becomes $\sum p_{j} \ln \left(\frac{p_{j}}{s_{i}}\right)$.

Expressed in this fashion, it is clear that both $T$ and MLD measure the difference in (log) shares of health (or ill health) and population, but $T$ is 
Figure A3. Trends in Mortality from Colorectal Cancer by Race, Ages 45-64, 1990-2001

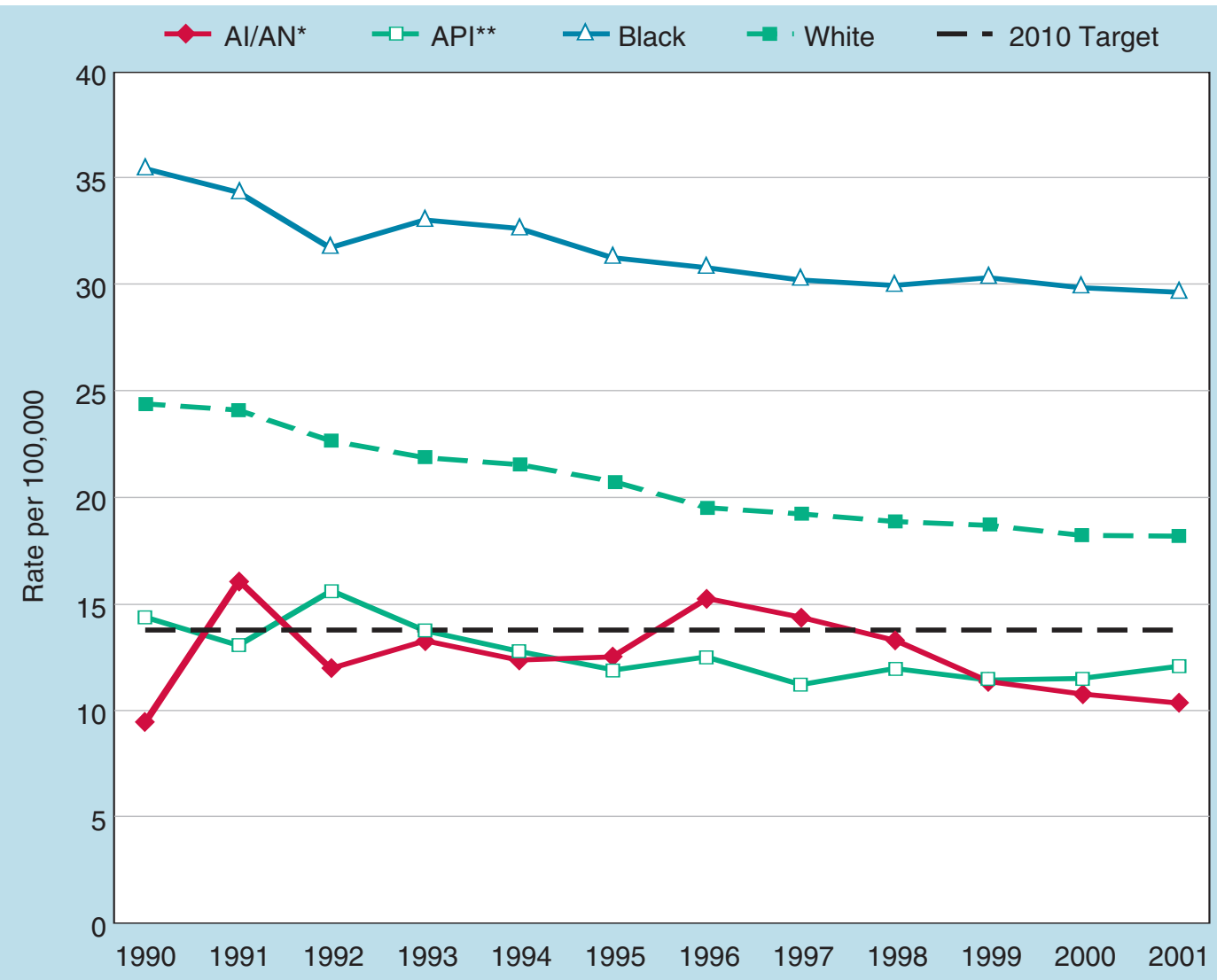

${ }^{*} \mathrm{AI} / \mathrm{AN}=$ American Indian/Alaska Native

**API = Asian/Pacific Islander

Source: Surveillance, Epidemiology, and End Results (SEER) Program (www.seer.cancer.gov) SEER*Stat Database, National Cancer Institute, DCCPS, Surveillance Research Program, Cancer Statistics Branch, released December 2003.

weighted by shares of health (or ill health, i.e., $s_{j}$ ), whereas $M L D$ is weighted by population shares $\left(p_{j}\right)$. Thus, in the context of our example $T$ will be somewhat more influenced by groups with high mortality ratios, whereas MLD will be somewhat more influenced by groups with large population shares.

Applying the disproportionality functions for $T$ and MLD listed in Table 2, in 1990 the American Indian/Alaska Native segment of $T$ is calculated as $0.006 \times 0.375 \times \ln (0.375)=$ -0.0023 , and the segment of MLD is $0.006 \times$ $[-\ln (0.375)]=0.062$. The Between-Group Variance $(B G V)$ is calculated using equation [17] above and, for the American Indian/Alaska Native segment in 1990 , is calculated as $0.006(9.5-25.5)^{2}=1.607$. The racial disparity in 1990 as measured by the Theil index is 0.0124 . We might then immediately ask whether this disparity is large or small. Because there has been little use of entropy-based measures in health disparities research, this is a difficult question to answer. We may get some leverage, perhaps, from the recent work by Goesling and Firebaugh (101), who used $T$ and $M L D$ to investigate trends in international health 
disparity in life expectancy among 169 countries. They report that in $2000 T$ for global disparity in life expectancy was 0.0099 and MLD was 0.0106 . We find a $T$ of 0.0198 and MLD of 0.0186 in 2002. Thus, it would appear that U.S. racial disparities in colorectal cancer among those 45-64 are similar in magnitude to cross-national disparities in life expectancy, but the extent to which the number of groups compared (4 race groups vs. 169 countries) and the health outcome (colorectal cancer mortality rates vs. life expectancy) may affect the level of disparity is an open question. As additional analyses applying entropy-based disparity measures within the United States are conducted (102), a clearer sense of how to interpret their magnitude should develop.
The results in Table A2 suggest that the absolute racial disparity $(B G V)$ in colorectal cancer mortality rates remained approximately constant over the period in question, but the relative disparity increased and to a slightly greater extent when measured with $T$ than with $M L D$. The slight difference between $T$ and MLD results from the fact that $T$ is somewhat more affected by high mortality ratios, and MLD is somewhat more affected by large population shares (88). Thus, in the case of colorectal cancer mortality, the Theil will be more sensitive to mortality change in blacks, and the MLD will be more sensitive to mortality change in whites. The black mortality ratio increased from 1.41 to 1.59 from 1990 to 2001, but the white ratio declined only slightly,

\section{Table A2. Example of Theil Index and the Between-Group Variance Applied to the Change in Racial Disparity in Colorectal Cancer Mortality, 1990 and 2001}

\begin{tabular}{|c|c|c|c|c|c|c|}
\hline Race & $\begin{array}{l}\text { Rate per } \\
100,000 \\
{\left[\mu_{j}\right]}\end{array}$ & $\begin{array}{l}\text { Population } \\
\text { Share } \\
{\left[p_{j}\right]}\end{array}$ & $\begin{array}{l}\text { Rate Relative } \\
\begin{aligned} \text { to } & T \text { otal } \\
& {\left[r_{j}\right] }\end{aligned}\end{array}$ & $\left.\begin{array}{cc} & T \\
{\left[p_{j} \times\right.} & r_{j} \times \ln ( \\
r_{j}\end{array}\right]$ & $\left.\stackrel{\operatorname{MLD}}{\left[_{j} \times-\ln (\right.} r_{j}\right]$ & $\begin{array}{c}B G V \\
p_{j}\left[\left(\mu_{j}-\Sigma \mu_{j}\right)^{2}\right]\end{array}$ \\
\hline \multicolumn{7}{|l|}{1990} \\
\hline $\begin{array}{l}\text { American Indian/ } \\
\text { Alaska Native }\end{array}$ & 9.5 & 0.006 & 0.375 & -0.0023 & 0.0062 & 1.607 \\
\hline Asian/Pacific Islander & 14.5 & 0.026 & 0.570 & -0.0084 & 0.0147 & 3.130 \\
\hline Black & 35.9 & 0.100 & 1.412 & 0.0486 & -0.0344 & 10.979 \\
\hline White & 24.7 & 0.868 & 0.970 & -0.0255 & 0.0263 & 0.502 \\
\hline Total & 25.5 & & & 0.0124 & 0.0128 & ${ }_{16.219}$ \\
\hline \multicolumn{7}{|l|}{2001} \\
\hline \multicolumn{7}{|l|}{ American Indian/ } \\
\hline Asian/Pacific Islander & 12.1 & 0.040 & 0.629 & -0.0116 & 0.0184 & 2.018 \\
\hline Black & 30.0 & 0.109 & 1.559 & 0.0751 & -0.0482 & 12.561 \\
\hline White & 18.3 & 0.843 & 0.950 & -0.0410 & 0.0431 & 0.776 \\
\hline Total & 19.2 & & & 0.098 & 0.0186 & ${ }_{16.027}$ \\
\hline
\end{tabular}


from 0.97 to 0.95 , thus favoring a relatively larger increase in the Theil index.

Figure A4 shows the absolute and relative disparity trends across the entire 11-year period, which indicate that the change in racial disparity was not constant over time. Both absolute and relative disparity declined from 1990 to 1992 , after which absolute disparity rose to remain at roughly its 1990 level for the rest of the period, while relative disparity continued to increase steadily as mortality rates declined for all racial groups. On the whole, then, we would take a less favorable view of the trend in racial disparities in colorectal cancer compared to that of educationrelated disparities in mammography. There virtually was no decline in absolute disparity, and relative disparity increased markedly from 1990 to 2002 despite an overall decline in the population mortality rate.

\section{Figure A4. Racial Disparity Trends in Working Age (45-64) Mortality from Colorectal Cancer by Race, 1990-2001}

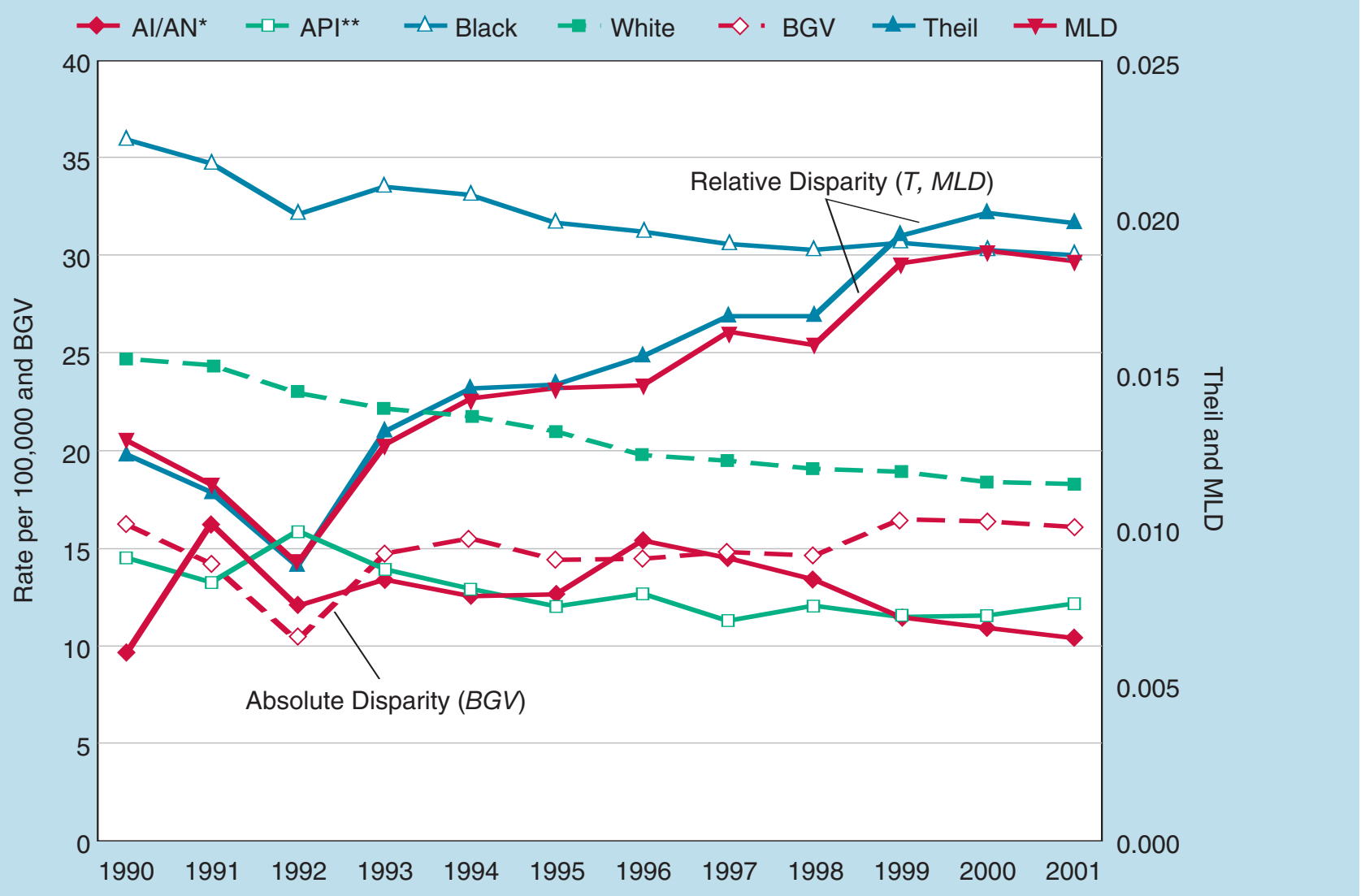

${ }^{*} \mathrm{AI} / \mathrm{AN}=$ American Indian/Alaska Native

**API = Asian/Pacific Islander

Source: Surveillance, Epidemiology, and End Results (SEER) Program (www.seer.cancer.gov) SEER*Stat Database: Mortality—All COD, Total U.S. for Expanded Races (1990-2001), National Cancer Institute, DCCPS, Surveillance Research Program, Cancer Statistics Branch, released December 2003. Underlying mortality data provided by NCHS (www.cdc.gov/nchs). 


\section{References}

1. U.S. Department of Health and Human Services. Healthy People 2010: Understanding and Improving Health. Washington, DC: U.S. Department of Health and Human Services, 2000.

2. Haynes MA, Smedley BD, Institute of Medicine, Committee on Cancer Research among Minorities and the Medically Underserved. The Unequal Burden of Cancer: An Assessment of NIH Research and Programs for Ethnic Minorities and the Medically Underserved. Washington, DC: National Academy Press, 1999.

3. U.S. Office of the Assistant Secretary for Health. Healthy People: The Surgeon General's Report on Health Promotion and Disease Prevention. Washington, DC: U.S. Department of Health, Education, and Welfare, Public Health Service, Office of the Assistant Secretary for Health and Surgeon General, 1979. DHEW (PHS) Publication No. 79-55071: DHEW Publication No. (PHS) 79-55071.

4. U.S. Public Health Service. Promoting Health/ Preventing Disease: Objectives for the Nation. Washington, DC: U.S. Department of Health and Human Services, Public Health Service, 1980.

5. U.S. Public Health Service. Healthy People 2000: National Health Promotion and Disease Prevention Objectives. Washington, DC: U.S. Department of Health and Human Services, Public Health Service, 1991. DHHS Publication No. (PHS) 91-50213.

6. Arizona Department of Health Services. Healthy Arizona 2010: Collaborating for a Healthier Future. Phoenix, AZ: Arizona Department of Health Services, 2001.

7. Iowa Department of Public Health. Healthy Iowans 2010: Advancing the Boundaries of Healthy Living and the Quality of Life in the New Decade. Available from URL: http://www.idph.state.ia.us/cpp/ healthy_iowans_2010.asp.
8. Lincoln-Lancaster Health Department. Healthy People 2010: Health Objectives for the Year 2010 for Lincoln \& Lancaster County, Nebraska. Lincoln, NE: Lincoln-Lancaster Health Department, 2000.

9. U.S. Department of Health and Human Services. Healthy People 2010. Conference Edition. Washington, DC: U.S. Department of Health and Human Services, 2000.

10. Poole C. Origins of risk relativism. Am J Epidemiol 2000;151(11):S46.

11. Spasoff RA. Epidemiologic Methods for Health Policy. New York: Oxford University Press, 1999.

12. Rothman KJ, Greenland S. Modern Epidemiology. 2nd Edition. Philadelphia, PA: Lippencott-Raven Publishers, 1998.

13. Szklo M, Nieto FJ. Epidemiology: Beyond the Basics. Gaithersburg, MD: Aspen, 2000.

14. Berkman LF, Kawachi I. Social Epidemiology. New York: Oxford University Press, 2000.

15. Krieger N, Fee E. Measuring social inequalities in health in the United States: A historical review, 1900-1950. Int J Health Serv 1996;26(3):391-418.

16. Krieger N, Chen JT, Ebel G. Can we monitor socioeconomic inequalities in health? A survey of U.S. health departments' data collection and reporting practices. Public Health Rep 1997;112(6): 481-91.

17. Krieger N. Socioeconomic data in cancer registries. Am J Public Health 2001;91(1):156-7.

18. Kitagawa EM, Hauser PM. Differential Mortality in the United States: A Study in Socioeconomic Epidemiology. Cambridge, MA: Harvard University Press, 1973. 
19. Pappas G, Queen S, Hadden W, Fisher G. The increasing disparity in mortality between socioeconomic groups in the United States, 1960 and 1986 [published erratum appears in NEngl J Med 1993;329(15):1139]. NEngl J Med 1993;329(2):103-9.

20. Duleep HO. Measuring socioeconomic mortality differentials over time. Demography 1989;26(2): 345-51.

21. Schalick LM, Hadden WC, Pamuk E, Navarro V, Pappas $\mathrm{G}$. The widening gap in death rates among income groups in the United States from 1967 to 1986. Int J Health Serv 2000;30(1):13-26.

22. Hahn RA, Eaker E, Barker ND, Teutsch SM, Sosniak W, Krieger N. Poverty and death in the United States-1973 and 1991. Epidemiology 1995;6(5):490-7.

23. Feldman JJ, Makuc DM, Kleinman JC, Cornoni-Huntley J. National trends in educational differentials in mortality. Am J Epidemiol 1989;129(5):919-33.

24. Elo IT, Preston SH. Educational differentials in mortality: United States, 1979-85. Soc Sci Med 1996;42(1):47-57.

25. Illsley R, Le Grand J. The measurement of inequality in health. In: Health and Economics. Williams A, editor. London: Macmillan Press, 1986, 12-36.

26. Pamuk ER. Social class inequality in mortality from 1921 to 1972 in England and Wales. Pop Studies 1985;39(1):17-31.

27. Illsley R. Reducing health inequalities: Britain's latest attempt. Health Aff 1999;18(3):45-6.

28. Hoyert DL, Singh GK, Rosenberg HM. Sources of data on socio-economic differential mortality in the United States. J Off Stat 1995;11(3):233-60.

29. Davis SK, Liu Y, Gibbons GH. Disparities in trends of hospitalization for potentially preventable chronic conditions among African Americans during the 1990s: Implications and benchmarks. Am J Public Health 2003;93(3):447-55.
30. Pearcy JN, Keppel KG. A summary measure of health disparity. Public Health Rep 2002;117(3): 273-80.

31. Keppel KG, Pearcy JN, Wagener DK. Trends in Racial and Ethnic-Specific Rates for the Health Status Indicators: United States, 1990-98. Hyattsville, MD: National Center for Health Statistics, 2002, (PHS) 2002-1237.

32. Singh GK, Miller BA, Hankey BF, Edwards BK. Area Socioeconomic Variations in U.S Cancer Incidence, Mortality, Stage, Treatment, and Survival, 1975-1999. Bethesda, MD: National Cancer Institute, 2003. NIH Publication No. 04-5417.

33. Krieger N, Chen JT, Waterman PD, Soobader M-J, Subramanian SV, Carson R. Geocoding and monitoring of U.S. socioeconomic inequalities in mortality and cancer incidence: Does the choice of area-based measure and geographic level matter? The public health disparities geocoding project. Am J Epidemiol 2002;156(5):471-82.

34. Singh GK, Miller BA, Hankey BF, Feuer EJ, Pickle LW. Changing area socioeconomic patterns in U.S. cancer mortality, 1950-1998: Part I-All cancers among men. J Natl Cancer Inst 2002; 94(12):904-15.

35. Singh GK, Miller BA, Hankey BF. Changing area socioeconomic patterns in U.S. cancer mortality, 1950-1998: Part II-Lung and colorectal cancers. J Natl Cancer Inst 2002;94(12):916-25.

36. Steenland K, Henley J, Thun M. All-cause and cause-specific death rates by educational status for two million people in two American Cancer Society cohorts, 1959-1996. Am J Epidemiol 2002;156(1):11-21.

37. Smedley BD, Stith AY, Nelson AR. Unequal Treatment: Confronting Racial and Ethnic Disparities in Health Care. Washington, DC: National Academy Press, 2003.

38. Weir HK, Thun MJ, Hankey BF, Ries LA, Howe HL, Wingo PA, Jemal A, Ward E, Anderson RN, Edwards BK. Annual report to the nation on the status of cancer, 1975-2000, featuring the uses of surveillance data for cancer prevention and control. J Natl Cancer Inst 2003;95(17):1276-99. 
39. Piffath TA, Whiteman MK, Flaws JA, Fix AD, Bush TL. Ethnic differences in cancer mortality trends in the U.S., 1950-1992. Ethn Health 2001;6(2): $105-19$.

40. Sen AK, Foster JE. On Economic Inequality. Expanded Edition. Oxford: Clarendon Press, 1997.

41. Temkin LS. Equality, priority or what? Econ Philos 2003;19(1):61-87.

42. Peter F, Evans T. Ethical dimensions of health equity. In: Evans T, Whitehead M, Diderichsen F, Bhuiya A, Wirth M, editors. Challenging Inequities in Health: From Ethics to Action. New York: Oxford, 2001, 25-33.

43. Whitehead M. The concepts and principles of equity and health. Int J Health Serv 1992;22(3): $429-45$.

44. Whitehead M, Dahlgren G. What can be done about inequalities in health? Lancet 1991; 338(8774):1059-63.

45. Carr-Hill R. Measurement Issues Concerning Equity in Health, Issues Panel for Equity in Health. London, UK: Kings Fund, 2001.

46. National Cancer Institute, Center to Reduce Cancer Health Disparities. How Are Cancer Health Disparities Defined? 2003. Available from URL: http://crchd.nci.nih.gov/chd/ disparities_defined.html.

47. $106^{\text {th }}$ Congress of the United States. Minority Health and Health Disparities Research and Education Act of 2000. 114 STAT. 2495-511. Public Law 106-525: 2000.

48. Satcher D. The history of the public health service and the surgeon general's priorities. Food Drug Law $J$ 1999;54(1):13-9.

49. Gakidou EE, Murray CJ, Frenk J. Defining and measuring health inequality: An approach based on the distribution of health expectancy. Bull World Health Organ 2000;78(1):42-54.

50. Murray CJ, Gakidou EE, Frenk J. Health inequalities and social group differences: What should we measure? Bull World Health Organ 1999;77(7):537-43.
51. Braveman P, Krieger N, Lynch J. Health inequalities and social inequalities in health. Bull World Health Organ 2000;78(2):232-4.

52. Asada Y, Hedemann T. A problem with the individual approach in the $\mathrm{WHO}$ health inequality measurement. Int J Equity Health 2002;1(1):2.

53. Braveman P, Starfield B, Geiger HJ, Murray CJL. World Health Report 2000: How it removes equity from the agenda for public health monitoring and policy commentary: Comprehensive approaches are needed for full understanding. BMJ 2001; 323(7314):678-81.

54. Houweling TAJ, Kunst AE, Mackenbach JP. World Health Report 2000: Inequality index and socioeconomic inequalities in mortality. Lancet 2001;357(9269):1671-2.

55. Walter SD. Choice of effect measure for epidemiological data. J Clin Epidemiol 2000;53(9): 931-9.

56. Sen AK. Inequality Reexamined. Cambridge, MA: Harvard University Press, 1992.

57. Bach PB, Cramer LD, Warren JL, Begg CB. Racial differences in the treatment of early-stage lung cancer. N Engl J Med 1999;341(16):1198-205.

58. Breen N, Wagener DK, Brown ML, Davis WW, Ballard-Barbash R. Progress in cancer screening over a decade: Results of cancer screening from the 1987, 1992, and 1998 National Health Interview Surveys. J Natl Cancer Inst 2001;93(22): 1704-13.

59. Hobbs F, Stoops N. Demographic Trends in the 20th Century. Census 2000 Special Reports, Series CENSR-4. Washington, DC: U.S. Government Printing Office, U.S. Census Bureau, 2002.

60. Wagstaff A, Paci P, van Doorslaer E. On the measurement of inequalities in health. Soc Sci Med 1991;33(5):545-57.

61. Pigou AC. Wealth and Welfare. London, UK: Macmillan, 1912.

62. Dalton H. The measurement of the inequality of incomes. Econ J 1920;30(119):348-61. 
63. Silber J. Health and inequality. Some applications of uncertainty theory. Soc Sci Med 1982;16(19): 1663-6.

64. Le Grand J. Inequalities in health: Some international comparisons. Eur Econ Rev 1987; 31(1-2):182-91.

65. Navarro V. World Health Report 2000: Responses to Murray and Frenk. Lancet 2001;357(9269): 1701-2.

66. Wolfson M, Rowe G. On measuring inequalities in health. Bull World Health Organ 2001;79(6): 553-60.

67. Wagstaff A, Van Doorslaer E. Overall versus socioeconomic health inequality: A measurement framework and two empirical illustrations. Health Econ 2004;13:297-301.

68. Gakidou E, King G. Measuring total health inequality: Adding individual variation to grouplevel differences. Int J Equity Health 2002;1(1):3.

69. Shkolnikov VM, Andreev EE, Begun AZ. Gini coefficient as a life table function: Computation from discrete data, decomposition of differences and empirical examples. Demographic Research 2003;8(11):305-57.

70. Kunst AE, Mackenbach JP. Measuring Socioeconomic Inequalities in Health. Copenhagen, Denmark: World Health Organization, Regional Office for Europe, 1995.

71. Ries LAG, Eisner MP, Kosary CL, Hankey BF, Miller BA, Clegg L, Edwards BK, editors. SEER Cancer Statistics Review, 1973-1999. Bethesda, MD: National Cancer Institute, 2002. http://seer.cancer.gov/csr/1973_1999.

72. Mackenbach JP, Cavelaars AEJM, Kunst AE. Socioeconomic inequalities in morbidity and mortality in Western Europe-reply. Lancet 1997;350(9076):517-8.

73. Vågerö D, Erikson R. Socioeconomic inequalities in morbidity and mortality in Western Europe. Lancet 1997;350(9076):516.
74. Mackenbach JP, Kunst AE. Measuring the magnitude of socio-economic inequalities in health: An overview of available measures illustrated with two examples from Europe. Soc Sci Med 1997;44(6):757-71.

75. Kunst A, Geurts J, van den Berg J. International variation in socioeconomic inequalities in selfreported health. J Epidemiol Community Health 1995;49(2):117-23.

76. Manor O, Matthews S, Power C. Comparing measures of health inequality. Soc Sci Med 1997;45(5):761-71.

77. Backlund E, Sorlie PD, Johnson NJ. The shape of the relationship between income and mortality in the United States. Evidence from the National Longitudinal Mortality Study. Ann Epidemiol 1996;6(1):12-20.

78. Preston SH, Haines MR, Pamuk E. Effects of industrialization and urbanization on mortality in developed countries. In: International Union for the Scientific Study of Population. International Population Conference, Manila, 1981: Solicited Papers. Vol. 2. Leige: Ordina Editions, 1981, 233-54.

79. Pamuk ER. Social-class inequality in infant mortality in England and Wales from 1921 to 1980. Eur J Popul 1988;4:1-21.

80. Liberatos P, Link BG, Kelsey JL. The measurement of social-class in epidemiology. Epidemiologic Rev 1988;10:87-121.

81. Kunst AE, Mackenbach J. International variation in the size of mortality differences associated with occupational status. Int J Epidemiol 1994;23(4): 742-50.

82. Duncan OD, Duncan B. A methodological analysis of segregation indices. Am Sociol Rev 1955;20:210-17.

83. Gaswirth JL. A graphical summary of disparities in health care and related summary measures. Unpublished manuscript (National Cancer Institute). 2003. 
84. Chakravarty SR. The variance as a subgroup decomposable measure of inequality. Soc Indic Res 2001;53(1):79-95.

85. U.S. Department of Health and Human Services. HHS Awards \$85 Million to Eliminate Health Disparities. Medical Research, Community Programs Are High Priorities. Rockville, MD: U.S. Department of Health and Human Services, 2002.

86. LaVeist TA. Measuring disparities in healthcare quality and utilization. In: Swift EK, editor. Guidance for the National Healthcare Disparities Report. Washington, DC: National Academy Press, 2002, 75-98.

87. Firebaugh G. Empirics of world income inequality. Am J Sociol 1999;104(6):1597-630.

88. Firebaugh G. The New Geography of Global Income Inequality. Cambridge, MA: Harvard University Press, 2003.

89. Reardon SF, Firebaugh G. Measures of multigroup segregation. Sociol Methodol 2002;32:33-67.

90. Theil H. Economics and Information Theory. Amsterdam: North-Holland, 1967.

91. Calle EE, Rodriguez C, Walker-Thurmond K, Thun MJ. Overweight, obesity, and mortality from cancer in a prospectively studied cohort of U.S. adults. NEngl J Med 2003;348(17):1625-38.

92. Rodriguez C, Patel AV, Calle EE, Jacobs EJ, Chao A, Thun MJ. Body mass index, height, and prostate cancer mortality in two large cohorts of adult men in the United States. Cancer Epidemiol Biomarkers Prev 2001;10(4):345-53.

93. Kakwani N, Wagstaff A, Vandoorslaer E. Socioeconomic inequalities in health: Measurement, computation, and statistical inference. J Econom 1997;77(1):87-103.

94. Dorschner J. The minority health crisis. Miami Herald. 13 September 2004; 22G.

95. Wagstaff A. The bounds of the concentration index when the variable of interest is binary, with an application to immunization inequality. Health Econ 2005;14:429-32.
96. Wagstaff A. Inequality aversion, health inequalities and health achievement. J Health Econ 2002;21(4):627-41.

97. Shorrocks AF. The class of additively decomposable inequality measures. Econometrica 1980;48(3):613-26.

98. Cowell FA, Jenkins SP. How much inequality can we explain? A methodology and an application to the United States. Econ J 1995;105(429):421-30.

99. Nelson DE, Powell-Griner E, Town M, Kovar MG. A comparison of national estimates from the National Health Interview Survey and the Behavioral Risk Factor Surveillance System. Am J Public Health 2003;93(8):1335-41.

100. Zhang Q, Wang Y. Socioeconomic inequality of obesity in the United States: Do gender, age, and ethnicity matter? Soc Sci Med 2004;58(6): 1171-80.

101. Goesling B, Firebaugh G. The trend in international health inequality. Popul Dev Rev 2004;30(1):131-46.

102. Sehili S, Elbasha EH, Moriarty DG, Zack MM. Inequalities in self-reported physical health in the United States, 1993-1999. Health Econ 2005;14(4):377-89. 